\title{
From Fabrics to Island Connections: Macroscopic and Microscopic Approaches to the Prehistoric Pottery of Antikythera
}

\author{
A. Pentedeka, E. Kiriatzi, L. Spencer, A. Bevan and J. Conolly \\ Postprint of a 2010 paper in the Annual of the British School at Athens 105: 1-81 (doi: \\ 10.1017/S0068245400000368).
}

\begin{abstract}
An intensive archaeological survey covering the entire extent of the island of Antikythera has recently revealed a sequence of prehistoric activity spanning the later Neolithic to Late Bronze Age, with cultural affiliations that variously link its prehistoric communities with their neighbours to the north, south and east. Here we present and discuss the results of a programme of both macroscopic and petrographic study of the prehistoric ceramics from Antikythera that defines a varied group of fabrics and explores their implications with regard to regional potting traditions, onisland production versus imports, and changing patterns of human activity on the island through time.
\end{abstract}

\section{INTRODUCTION}

This paper considers prehistoric pottery from an intensive survey of the Greek island of Antikythera, with particular emphasis on the interpretative advantages offered by a combined macroscopic and microscopic approach. Antikythera is one of the smallest (20.8 sq.km, maximum elevation $379 \mathrm{~m}$ ) and more remote inhabited islands in the Mediterranean, but one well-placed to benefit from routes of maritime travel from Crete to the Peloponnese and from the central Mediterranean to the Aegean. It has experienced an eventful history of human exploitation beginning some 7,000 years ago, with evidence for a broad range of activities from seasonal visitation to settled agriculture to piracy (e.g. Bevan et al 2008). Until 2005, its prehistory was almost entirely unknown, but recent archaeological survey (the Antikythera Survey Project, ASP $)^{1}$ was able to cover the island's entire extent using intensive methods and has

${ }^{1}$ We would like thank the Greek Ministry of Culture, the Greek Archaeological Service (26th EPKA, 1st EBA) and three main external funding agencies over the duration of the survey project-the Social Science and Humanities Research Council of Canada, the UK Arts and Humanities Research Council and the Institute for Aegean Prehistory. The survey's sponsor in Athens was the Canadian Institute in Greece, and we are especially grateful to Jonathan Tomlinson for his assistance. The petrographic analysis discussed here was undertaken at the Fitch Laboratory, British School of Athens with the help of a British Academy small grant (SG-45163) and we would like to thank both of these institutions for their support. The co-director and synergast for the Antikythera Survey Project was Aris Tsaravopoulos, and we could not have undertaken this research without his generous assistance and guidance. We are also extremely grateful to Cyprian Broodbank, co-director of the Kythera Island Project, who provided a wide range of discussion, advice and support from the 
revealed a series of different visitation and settlement episodes, as well as recovering a substantial assemblage of knapped stone and pottery for further study.

Of this prehistoric material, the pottery comprises about 6,500 sherds that were collected in three different ways (FIG.1; and for further details, see Bevan et al 2008 or the ASP website): (i) a first stage of survey in which teams of five people walked across the entire island in lines $15 \mathrm{~m}$ apart (leading to the collection of ca.500 prehistoric 'feature' sherds: i.e. rims, bases, handles and those with glaze, paint or other decoration), and a second stage in which we resurveyed promising prehistoric scatters on a 10x10 m grid, collecting (ii) all of the pottery that could be found inside a 5sq.m circular area within each grid square (ca.4,450 sherds), and (iii) all remaining feature sherds in the square (ca.1,550 sherds).

project's beginnings. In addition, Birgitta Hallager, Julie Hruby, Jenny Moody, Eleni Nodarou, Jerry Rutter, Cynthia Shelmerdine and Todd Whitelaw were also kind enough to offer their thoughts on particular issues or artefacts. Denitsa Nenova illustrated the sherds catalogued here, with further assistance by Marek Maciusowicz, James O'Neill and Natalie Willimott.

At the time of writing, the various digital ASP datasets can be accessed from the ASP website at either http://www.ucl.ac.uk/asp or http://www.tuarc.trentu.ca/asp and are being archived more permanently with the UK Archaeological Data Service (http://ads.ahds.ac.uk/).

The following abbreviations are used in this article:

Arf argillaceous rock fragment

ASP Antikythera Survey Project

ASPGS Antikythera Survey Project Geological Sample

CAL Calcite tempered fabric

CAS Calcareous and Sedimentary fabric

EB, EBA Early Bronze Age

EM Early Minoan

FN Final Neolithic

FPal First Palace period

GRO Grog tempered fabric

LM Late Minoan

LN Late Neolithic

LPrePal Late Prepalatial period

MIC Micaceous fabric

MM Middle Minoan

MUT Mudstone tempered fabric

OUT Outlier fabric

PPL plane polarised light

SAT Sand tempered fabric

SPal Second Palace period

Tcf textural concentration feature

TPal Third Palace period

UTM Universal Transverse Mercator coordinate system

XP crossed polarised light

WGS World Geodetic System 
This material therefore offers a systematic and spatially coherent picture of prehistoric activity on the island, but is challenging to analyse, because (i) the vast majority comprises coarsewares with little if any surviving decoration (>95\%), (ii) there is no excavated prehistoric site on the island to provide us with a local stratified sequence, and (iii) it is clear that many of the surface scatters on the island reflect more than one phase of prehistoric activity, making inter-scatter seriation of the shapes and fabrics more difficult. Despite these challenges, substantial insights can still be drawn from such surface material via the careful combination of macroscopic and microscopic methods of fabric analysis that we describe below.

The ASP prehistoric ceramic material catalogued below presents the petrographic characterisation of 175 prehistoric ceramics-and a small number of other, unsampled, sherds where these are particularly relevant to discussion. This paper is meant as the primary discussion of these fabric groups, but also offers a route into the comprehensive ASP pottery database, thin sections, digital photos and line drawings that are available online. ${ }^{2}$ The section below (2. Fabric analysis) begins by explaining the relationship between our macroscopic and microscopic investigative methods and is then followed by a consice summary of the petrographic analysis results. In the following section (3. Chronological comments) we summarise the results of macroscopic and microscopic approaches to the prehistoric pottery of Antikythera so as to create a 'ceramic profile' for each chronological phase, taking also into account the ceramic developments in neighbouring areas, and in particular Kythera and Crete. The final section (4. Discussion) addresses the wider implications of the chronological, technological and cultural features visible in the studied material and is then followed by a catalogue of the sampled sherds arranged by fabric groups (5. Catalogue).

\section{FABRIC ANALYSIS}

Due to the fact that traditional form- and decoration-based dating methods are notoriously difficult to apply to fragmentary and coarse surface survey material, it was necessary to employ alternative methodologies by which the pottery could be identified and dated. Given that Antikythera has no excavated prehistoric site on the island from which a stratified, form-based sequence might be developed, this further complicated the approaches that could viably be employed. Fortunately, the core elements of a systematic diachronic methodology to analyse survey pottery were developed in the 1980s by J. Moody and have been widely adopted across Crete (see Moody et al 2003, 39-44); more recently, this methodology has been further enhanced and successfully applied in the context of the Kythera Island Project (located on the nearby and larger island of Kythera) (Kiriatzi 2003, 124-126). The key to this methodology is the division of ceramics into macroscopic fabric groups based upon clay color, texture and inclusion composition (nature, size, roundness and sorting). By dividing the pottery into clusters relating to the raw materials from which they were produced, this creates a series of classifications that relate to either how or where the pottery was made. The second element of this methodology involves developing a basic chronology for each macroscopic fabric group through the identification of period-specific shapes in particular fabrics (known as 'index sherds'); this allows for non-diagnostic sherds in terms of shape to be given a date range due to their fabric

\footnotetext{
${ }^{2}$ See fn. 1.
} 
(see Moody et al 2003, 48-54, for a detailed description of this methodology in the context of the Sphakia Survey).

The combination of macroscopic fabric classification and chronological-shape associations has proved to be key to exploring the prehistoric pottery of Antikythera, despite the fact that the published information on the chronological variation amongst coarse wares of the southern Aegean (particularly in western Crete) is limited. For this reason, the analysis of the Antikythera pottery has benefited greatly from close collaboration and comparison of material with the Kythera Island Project, for which a prehistoric chronology has been developed that is arguably one of the most effective in the Mediterranean to date. Therefore, the Antikythera material has been dated through a combination of (i) general typological features; (ii) comparisons with the typological and fabric-based sequence established by the Kythera Island Project on the neighbouring island of Kythera; (iii) comparisons with the typologies and fabrics of Cretan excavations and survey projects (particularly those in western Crete, e.g. Debla, Monastiraki and Nopigeia for the Prepalatial and First Palace period, Khania and Nerokourou for the Second Palace period as well as the Khania Area Survey and the Sphakia Survey; also, east Cretan surveys that utilise a fabric-oriented approach such as Vrokastro and Kavousi have been used for comparative purposes; for Debla see Warren \& Tzedakis 1974; for the Khania Area Survey Moody 1985, 1987, 2004; for the Sphakia Survey Moody et al 2003; for Vrokastro Moody 2005; for Kavousi Mook 2005).

The most striking aspect of the ASP prehistoric pottery macroscopic study is the diversity of fabrics found on Antikythera. A prolonged first season of pottery study in 2007 had as one of its main objectives the identification of macroscopic fabric groups. It suggested twelve such groups, plus a number of outliers, as follows:

1. Angular sand tempered: fine non-micaceous clay which fires red/orange to pinky buff, often with grey core. Frequent poorly sorted angular inclusions of chert, mudstone/siltstone and carbonates.

2. Rounded sand tempered: similar to 1 in inclusion types and frequency, but with differences in the inclusions' sphericity (rounded-well rounded) and sorting (well sorted), as well as the rather finer, cleaner clay.

3. Calcite tempered: divided into two macroscopic subgroups a) dense and red firing, with frequent dense angular limestone inclusions and b) buff-tan firing with frequent poorly sorted sub-angular limestone fragments and sporadic mudstone and chert inclusions.

4. Chert fabric: dense clay texture, inclusions of sparse angular chert, rare subrounded mudstone grains and other sporadic red to black inclusions that could be grog,

5. Grog tempered: soft pink to buff clay, frequent inclusions of small to large grog fragments, small angular chert grains and rare mudstone plates.

6. Red micaceous: inclusions of mica and mica-rich rock fragments in a red firing clay, very similar to the Kytheran Red micaceous fabric (Kiriatzi 2003, 125) 
7. Kytheran mudstone tempered: buff to orange clay, inclusions of angular mudstone and rare chert or carbonates, very similar to the Mudstone tempered fabric identified at Kythera (Kiriatzi 2003, 125).

8. Kytheran sand tempered: light pink firing colour, inclusions of dark grey to red mudstone, white to grey carbonates and chert. Different from 1 and 2 in frequency, size (smaller grains) and sorting of inclusions (well-sorted), being very similar to the sand-tempered fabric recognised at Kythera (Kiriatzi 2003, 125).

9. Mudstone tempered: silty red-firing clay, inclusions of very frequent small angular mudstone plates or angular mudstone fragments, frequent angular chert and rare angular white chert.

10. Shale: two macroscopically-identifiable varieties a) with common, dense platy grey and red shale inclusions and rare mica and quartz, and b) with red/brown and grey shale plates and common quartz, mica, siltstone and mudstone inclusions.

11. Angular mudstone and chert: lightly micaceous fine dense clay, inclusions of rare large angular mudstone and chert.

12. Fine: fine well-levigated clay, very few inclusions macroscopically visible. Unlikely that pottery from this group derives from a single source, impossible to make further divisions due to fine texture and lack of inclusions.

This early phase of analysis also made it very clear that the first two sand-tempered groups described above were by far the most common amongst the Antikythera material, followed by those with grog tempering and with other groups each making up a much lower proportion of the overall assemblage. The above macroscopic fabric sequence of the prehistoric ceramics from Antikythera was also then used as the basis for carefully selecting 175 samples for petrographic analysis. The microscopic analysis of these samples that followed therefore aimed to:

(i) verify the reality of macroscopically identified fabric groups;

(ii) identify the major mineralogical components of each fabric group in order to determine their compatibility with local geologies or to suggest their off-island origin;

(iii) explore variation within and across these fabric groups stemming from production decisions (technological choices), and

(iv) at a broader level, offer further insights about on-island potting strategies and possible changing patterns of cultural interaction between Antikythera, Kythera, the Greek mainland and Crete during the latest Neolithic through to the Late Bronze Age, for which there are growing sets of comparable petrographic data.

All samples were subjected to petrographic analysis with thin sections (using a Leitz Laborlux 12 POL polarising microscope), with the aim of characterising both their mineralogical composition and texture so as to assess possible geological provenance, explore possible clay paste preparation technologies (i.e. clay tempering or mixing), and understand prehistoric pot-firing conditions. With regard to the latter, the samples were subjected to refiring tests: chips from all the samples were refired at $1000^{\circ} \mathrm{C}$ in 
oxidising conditions using a Naberthem L5/P furnace. ${ }^{3}$ Refiring of the ceramic samples in fully oxidising conditions, at higher temperature (as estimated on the basis of thin section exanination) and possibly longer duration, helps distinguish different clay compositions reflected in colour, by eliminating any colour variation in the samples that was caused by the original firing conditions. Detailed microscopic descriptions follow the descriptive system proposed by Whitbread (Whitbread 1986, 1989, 1995). The assessment of possible fabric group provenance was aided enormously by the comparative pottery and geological samples collection in the Fitch Laboratory from Kythera (recovered by the Kythera Island Project: Kiriatzi 2003) and from west Crete (from various sources: Chandler 2001), as well as by further consultation with other colleagues working on the fabrics and/or ceramic petrography of western Crete (e.g. E. Nodarou; P.M. Day; J. Moody).

Familiarity with the geological background of Antikythera is important in order to assess compositional and textural attributes detected in pottery. In broad outline, the geology of Antikythera is characterised by Quaternary deposits, along with formations belonging to the Tripolis geological zone. The island bedrock consists of rudistbearing limestones of the Upper Cretaceous, strongly dolomitised and micritic. Superimposed, nummulitic limestones of Paleocene-Middle Eocene date are found, along with restricted areas of Upper Eocene flysch, consisting of alternations of siltstones and shales with sandstone layers. Quaternary formations consist mainly of Holocene deposits (terra rossa, carbonate scree) and Plio-Pleistocene clastic carbonate series (calcarenites and calc-rudites, along with small quantities of cherty, quartzose, quartzitic and arenaceous clastic material, as well as breccia-conglomerates). Neogene formations comprise marls, sandstones and conglomerates, having been deposited in two main tectonic areas in the western and central part of the island (Galeos \& Drandaki 1993).

Limited raw material sampling was also carried out, with particular regard to sandtempered fabrics. Three sand samples and one chert rock fragment were collected from the north-central part of the island (the beach deposits at Potamos-Chalara and Xeropotamos for the sand samples and the Charchaliana area for the chert rock fragment). The sand samples (ASPGS1-ASPGS3) derive from the Neogene formations and contain variable amounts of limestone, chert, quartzite, mudstone and sandstone rock fragments, whereas ASPGS4 is secondary chert rock fragment, originating from the Upper Cretaceous fossiliferous limestone rock formation.

Overall, the macroscopic groupings proposed by preliminary study proved to match well with those that were later identified under the microscope, with the exception of the fine fabric (12 above), for which petrographic fabric characterisation was not feasible because at this stage the sampling strategy focused on coarse fabrics. Therefore, only one sample (catalogue number 172) represents this macroscopic group, allowing for no further discussion of this, in other respects, well-identified fabric. With regard to the remaining eleven macroscopically identified groups, eight were proved valid via petrographic analysis while three (nos. 2, 4 and 11 as listed above) proved to be rather similar to other identified microscopic fabric groups and were, therefore, incorporated into them. More precisely, the majority of rounded sand tempered and angular mudstone and chert groups ( 2 and 11 above respectively) go

\footnotetext{
${ }^{3}$ Maximum temperature was achieved gradually in 2 hours time and was kept stable for 1 hour, after which the kiln was turned off and the samples were left to cool overnight.
} 
well with the general sand tempered microscopic groupings, whereas most of the samples initially identified as a chert group (4 above) fall into microscopic groups that were better classed as grog tempered.

What follows is a concise presentation of the final fabric groupings produced after petrographic analysis, along with a short discussion on their correlation to the initial macroscopic categories. Petrographic analysis of the prehistoric pottery from Antikythera distinguished seven major fabric groups, described below, the majority of which could be further subdivided depending on compositional and/or textural variability. Alongside fabric descriptions, basic information on vessel shape is given and further reference can thereafter be made to the accompanying line drawings and photographs. Only one representative thin section photograph per fabric group is published here (PLATE 1), but a complete set is available online or from the authors on request. A full catalogue of the sampled sherds can be found in section 5. Sherds have been given a single running number sequence throughout this article that is shown in boldface (e.g. 1), but readers should also take note of the associated petrographic sample numbers (e.g. ASP1) and more complex field identifiers in brackets (e.g. 2021-8-1-95-1), as the latter are the ones physically marked on the finds or finds bags and used in other ASP publications. A location for each potsherd is provided in metres (UTM zone 34N, WGS84, see figure 1), with a working accuracy that is typically to the nearest $10 \mathrm{~m}$. All other measurements are in centimetres. The chronological periods used below reflect the broad diagnostic categories with which surveys are typically forced to work. We have sought to ensure comparability with the divisions already in use for the pottery of the Kythera Island Project survey to the north and have adopted the following basic scheme (TABLE 1), only adding finer distinctions in cases where this seems justified: ${ }^{4}$

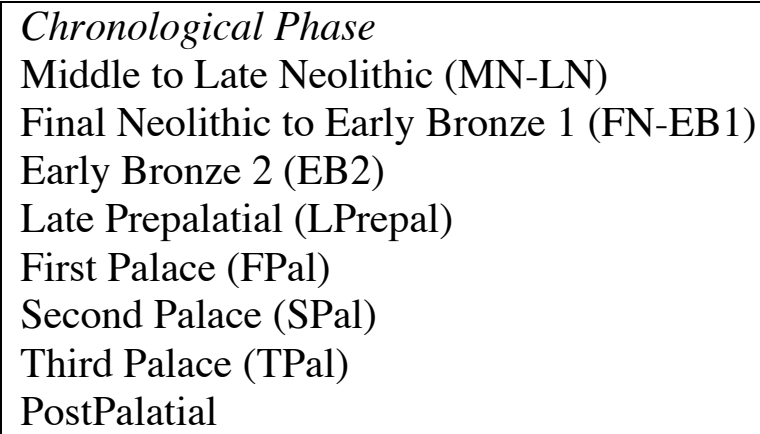

Absolute Dates (approx.)
$6000-4500 \mathrm{BC}$
$4500-2700 \mathrm{BC}$
$2700-2200 \mathrm{BC}$
$2200-1900 \mathrm{BC}$
$1900-1700 \mathrm{BC}$
$1700-1450 \mathrm{BC}$
$1450-1200 \mathrm{BC}$
$1200-1000 \mathrm{BC}$

\footnotetext{
${ }^{4}$ There are only minor differences from the Kythera Island Project scheme: Arabic numerals are preferred for the EB phases and 'Late Prepalatial is preferred to 'First Minoanizing'. Regarding the latter change, it was thought better not to incorporate this assumption of a well-timed shift from non-Cretan to Cretan cultural affiliation into ASP's basic chronology from the outset, because any such shift is either more complex or less well-identified archaeologically on Antikythera than it is on Kythera. Note that the Neolithic and Early Bronze Age periodisation is a generic Aegean one rather than tied directly to the Cretan scheme. Thereafter, the subsequent use of Cretan palatial periods is made for comparative convenience and does not automatically imply that political processes on Crete had immediate impacts on Antikythera.
} 
TABLE 1. The main chronological phases used by the Antikythera Survey Project.

Finally, it is necessary to explain briefly the rationale behind the way the sherds are dated in the catalogue below. During study of the material, and to enable certain kinds of statistical treatment thereafter, ASP adopted a fuzzy, belief-based, approach to recording the dateline of individual sherds. Rather than give them a categorical date in the usual way (e.g. sherd $\mathrm{x}$ is "Second Palace Period, or possibly Third Palace Period"), we offered a rough measure of our confidence that the sherd belonged to a certain phase (e.g. ca.70\% Second Palace Period, ca.30\% TPal Period). ${ }^{5}$ These percentages reflect the opinion of those who have studied the material, based on comparative evidence from neighbouring regions about changes in vessel shape, decoration and fabric over time. We have chosen to retain them here (in the fabric tables as bar charts and in the catalogue as raw percentages) in order to be consistent with other ASP publications. In the clearest-cut cases where the date of a sherd in the catalogue can be further narrowed down based on the overall chronology of the surface scatter from which it comes (i.e. those deemed to be single period assemblages), we have not incorporated this extra evidence in the percentages, but have given it separately in brackets afterwards.

\subsection{SAND TEMPERED FABRICS (SAT, and subgroups SATa, b, c, and d)}

Sand-tempered ceramics are by far the most common on Antikythera, comprising $48 \%$ of the overall prehistoric assemblage. Sand tempering is a tradition that is visible in Cretan pottery production from at least EMII (e.g. Wilson and Day 1994, 67-8, group 18; Whitelaw et al 1997, 268, n.21; Kiriatzi 2003, 129; Nodarou 2003), and is also common amongst Bronze Age fabrics in western Crete, particularly during the Second Palace period (Moody 1985, 56). On Antikythera, this broad date range is also evident and such fabrics are particularly common during the First and Second Palace periods. Sand-tempered fabrics cover a wide range of shapes, from large to small size vessels, and are associated with a variety of functions from storage to cooking and serving/consumption.

Macroscopic fabric analysis distinguished three sand-tempered groups: one of these (Kytheran sand tempered, no 8 above) was also identified as a separate group (SATd) by petrographic analysis, whereas the other two (angular sand tempered and rounded sand tempered, nos 1 and 2 above) were rather similar under the microscope. Equally, the macroscopic group angular mudstone and chert was incorporated into the wider class of sand-tempered fabrics.

Overall, SAT fabric is coarse, characterised by poorly sorted rounded to sub-angular inclusions, bimodal grain size distribution, with maximum grain size $4 \mathrm{~mm}$ (mode size $0.75-1.2 \mathrm{~mm}$ ) and $5-10 \%$ voids. The fine fraction consists predominantly of silicate inclusions (quartz/alkali feldspars, few chert fragments and plagioclase) in all samples, while the coarse fraction contains dominant to predominant chert rock

5 Chronological uncertainty has a huge effect on the interpretation of any archaeological pattern and such a belief-based (or loosely probabilistic) approach to artefact dating is an experiment that allows us to address the issue statistically, as well as measure other important factors, such as intra-and inter-observer variation in the dates assigned by those specialists who study the artefacts, or the gradual accumulation of extra chronological clarity that might come from laboratory study. 
fragments (deriving from both primary and secondary formations), few to common quartz, few to very few quartzite rock fragments, and few to very rare mudstone, sandstone, limestone and shale rock fragments. At least three main sand-tempered sub-groups can be identified on Antikythera, both macroscopically and petrographically (on the basis of frequency and grain size of the fine fraction inclusions, as well as groundmass optical activity), alongside others whose frequencies are too low to allow grouping at this stage. Several of the fabric subgroups are compositionally compatible with the local geology of Antikythera associated with chert and limestone formations of the Tripolis zone, and hence may have been made on the island, but similar formations are also found in western Crete and some may well have been made there, while at least one sub-group is demonstrably from Kythera to the north (SATd).

All samples of this fabric group are tempered with sand (rounded grain shape), for subgroups SATa-c of chert composition, for SATd of a more variable composition. Experimental replication of the recipe by adding crushed chert (ASPGS4) resulted in angular to subrounded fragment shapes, frequently conchoidal. These shapes thus differ from those found added as temper to the Antikytheran pottery, with the latter being rounded and probably from a natural sediment formation, i.e. sand. Sand samples examined (ASPGS1-ASPGS3) contain very similar chert fragments, along with very similar mudstone fragments, but in contrast also present a high frequency of calcareous fragments that is not observed in any pottery sample. Apart from tempering, clay mixing seems to be an equally popular technological choice, since a variety of clay pellets and streaks of different colour and/or texture are observed (e.g. 2, 29, 31, 34).

The general picture of this fabric is that of the application of a standardised technique (tempering) to a variety of claybases. Although the fabric's overall mineralogy is compatible with local geology, the observed variation in the clay base may reflect the use of different clay sources within, and most possibly beyond, the island (probably in western Crete).

\subsubsection{SATa (FIGS. 2-4)}

This is a coarse fabric, containing chert (frequently radiolarian, occasionally with chalcedony veins), mudstone and sandstone (also quartz, siltstone, Tcfs/Arfsmudstone, very rare grog and shale). The fine fraction contains silt-sized inclusions in an open to double-spaced porphyric related distribution, while chert fragments tend to have a more rounded grain shape in comparison to SATb. This composition is entirely compatible with the local geology on Antikythera, though a western Cretan provenance is also possible. The sampled sherds suggest a fabric that is rather homogeneous in terms of its composition and bimodal in its grain size distribution, although some variability is observed in the refiring colour. The latter variability might be due to the presence of secondary calcite in some of the samples, or to possible clay mixing in others. Overall, however, the main refiring colour is red, reflecting the use of similar types of clays, which are tempered primarily with subangular to rounded,, coarse sand-sized chert fragments. The optical activity of the groundmass (moderately to slightly inactive) suggests original firing temperatures around $800^{\circ} \mathrm{C}$. The firing atmosphere was oxidising to oxidising/reducing, while the common presence of a grey core possibly implies a rather short duration of firing. 


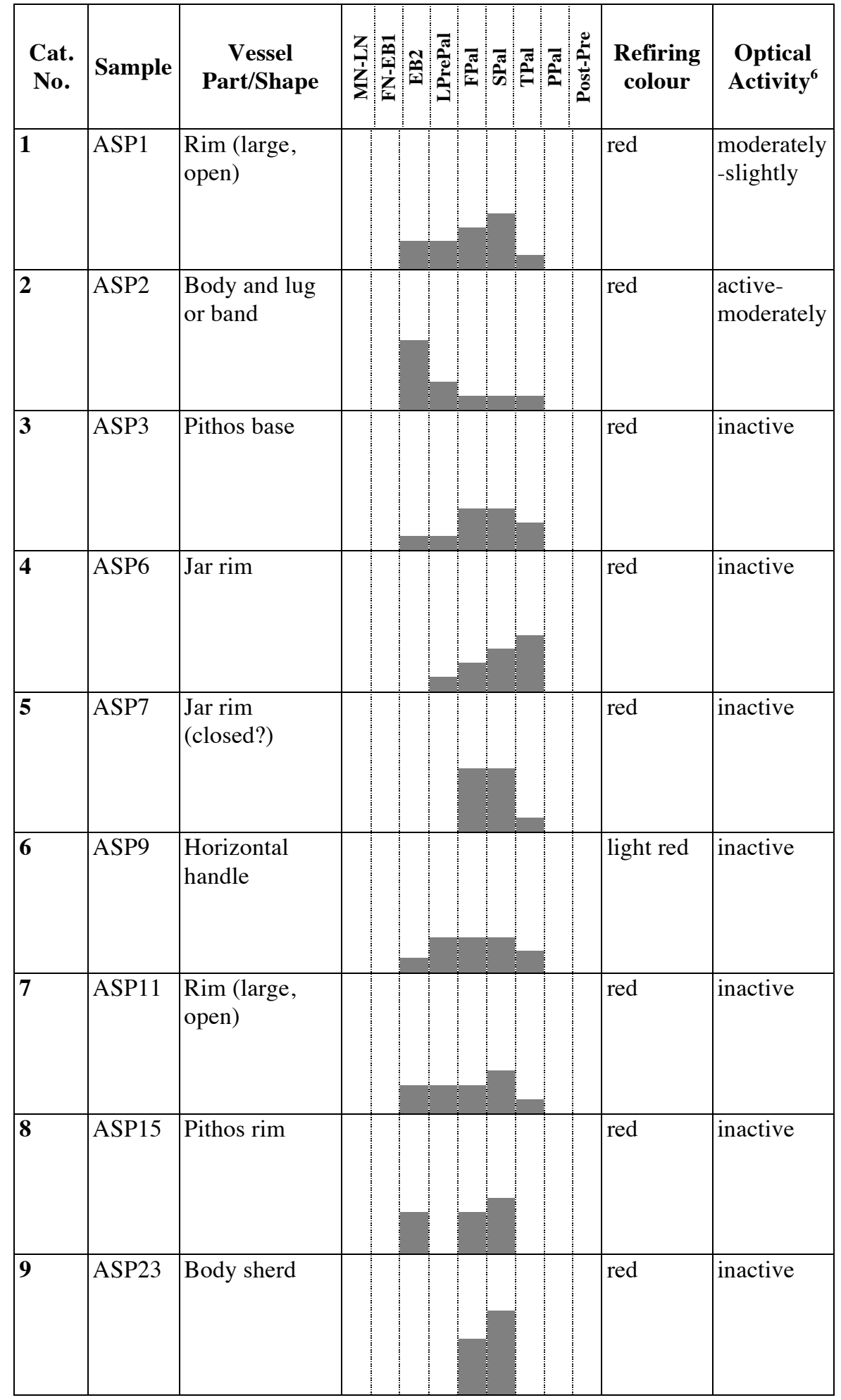

${ }^{6}$ Optical activity refers to the alteration clay minerals undergo due to firing. Modifiers are used to express the degree of this alteration and give information on the original firing temperatures: active $=$ no alteration, low-fired; inactive $=$ vitrified, high-fired (Whitbread 1995: 382, 394). 


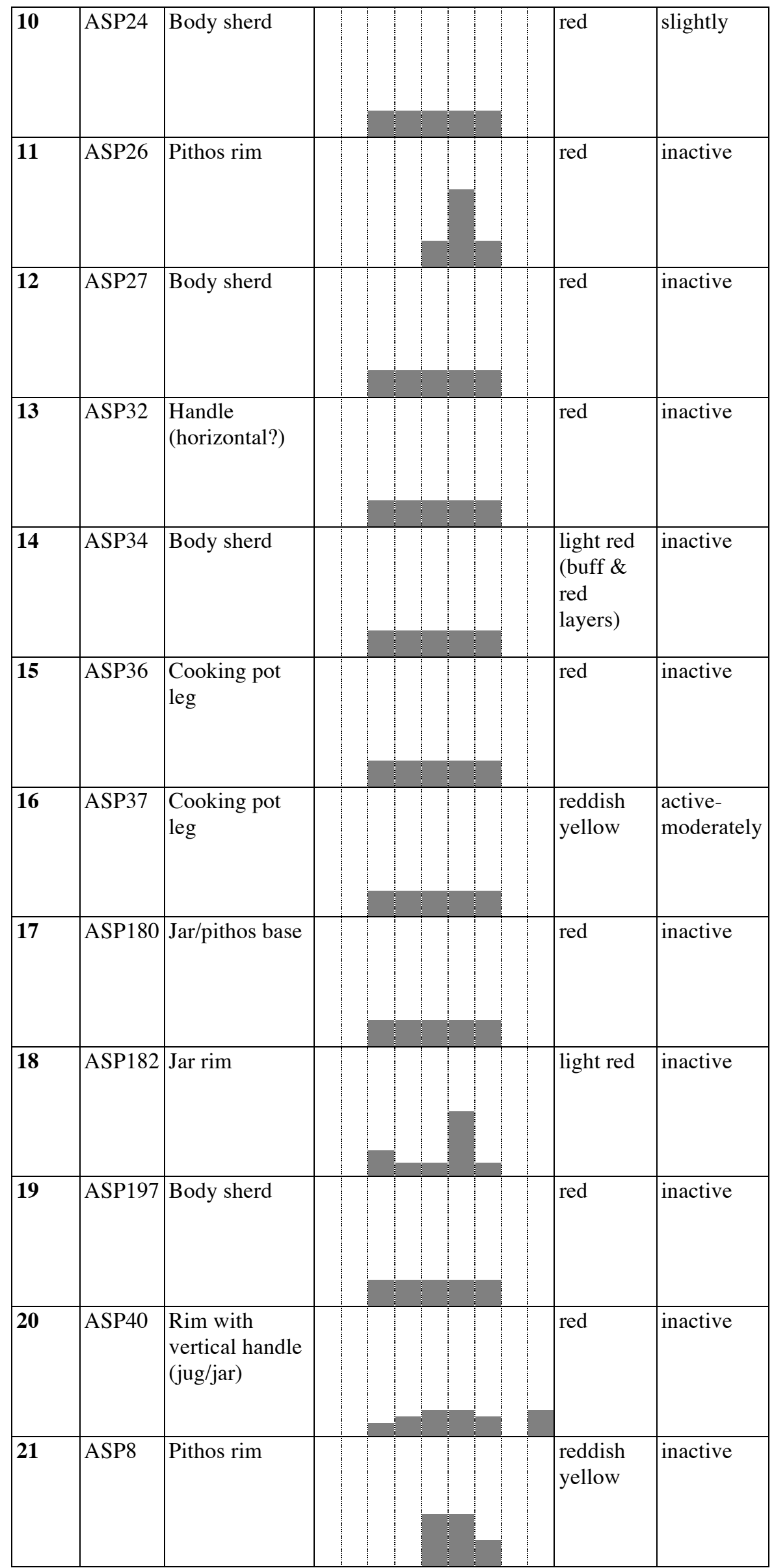




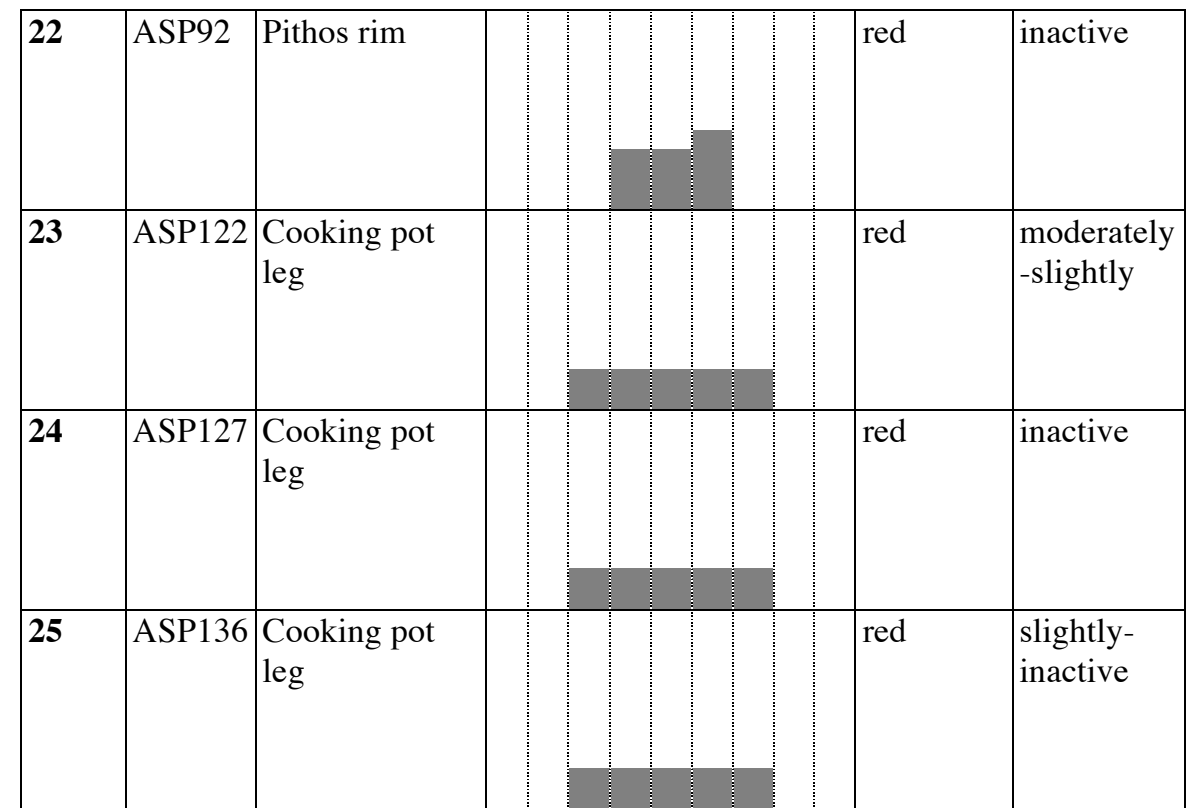

\subsubsection{SATb (FIGS. 5-6)}

This fabric subgroup does not present any clear compositional differences to SATa, but a distinction can be made on textural qualities, namely the grain size characterising the fine fraction that in this case consists of very fine-medium grain sand size silicate/quartz inclusions in an open to single-spaced porphyric related distribution. This might imply the use of a different clay base, which, nevertheless, refires the same red colour. This sub-group is compatible to local geology but might also have been made in western Crete.

\begin{tabular}{|c|c|c|c|c|c|c|c|}
\hline $\begin{array}{l}\text { Cat. } \\
\text { No. }\end{array}$ & Sample & $\begin{array}{c}\text { Vessel } \\
\text { Part/Shape }\end{array}$ & 妾离 & वิ) & क्षे: & 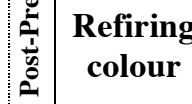 & $\begin{array}{l}\text { Optical } \\
\text { Activity }\end{array}$ \\
\hline 26 & ASP4 & Body sherd & & & & red & inactive \\
\hline 27 & ASP10 & $\begin{array}{l}\text { Rim (open, } \\
\text { shallow) }\end{array}$ & & & & $\begin{array}{l}\text { red (thin } \\
\text { buff } \\
\text { layer) }\end{array}$ & inactive \\
\hline 28 & ASP12 & Pithos base & & & & red & inactive \\
\hline 29 & ASP14 & $\begin{array}{l}\text { Rim (open, } \\
\text { shallow) }\end{array}$ & & & & light red & inactive \\
\hline
\end{tabular}




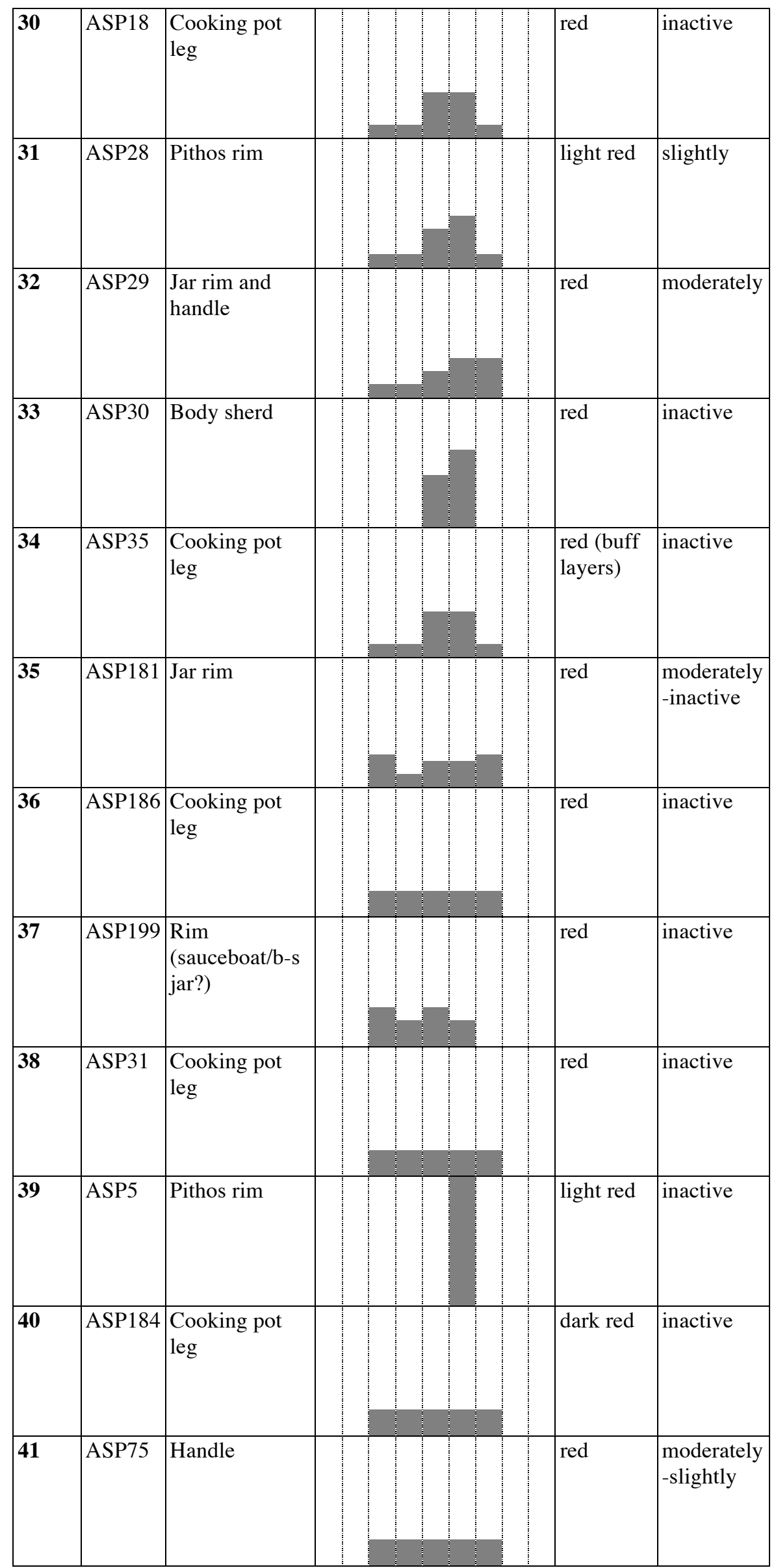






\subsubsection{SATC (FIG. 7)}

While there are hardly any significant compositional differences to SATa that can be observed in this sub-group apart from the lower frequency of inclusions in the coarse fraction and the existence high frequency Tcfs, probably clay pellets, except in $\mathbf{4 7}$ were they could be oxide products of serpentinite weathering), textural differences do exist. In particular, the groundmass of this group is noticeably finer-grained, indicating the use of a refined or naturally clean clay, which however refires red. One further difference is possibly a lower firing temperature (below/around $800^{\circ} \mathrm{C}$ ), indicated by the strong birefringence and optical activity of the groundmass (active). Its mineralogy is compatible to the local geology on Antikythera (and if locally produced, would therefore point to a diversification of the main fabric recipe), but could also have been made in western Crete.

\begin{tabular}{|c|c|c|c|c|c|c|c|c|c|c|c|}
\hline $\begin{array}{l}\text { Cat. } \\
\text { No. }\end{array}$ & Sample & $\begin{array}{c}\text { Vessel } \\
\text { Part/Shape }\end{array}$ & 紊 & $\overline{0}$ & & : & $\frac{\pi}{\pi}$ & ฝิ & હેّ & $\begin{array}{c}\text { Refiring } \\
\text { colour }\end{array}$ & $\begin{array}{l}\text { Optical } \\
\text { Activity }\end{array}$ \\
\hline 43 & ASP19 & $\begin{array}{l}\text { Handle or } \\
\text { cooking pot leg }\end{array}$ & & & & & & & & red & active \\
\hline 44 & ASP20 & $\begin{array}{l}\text { Cooking pot } \\
\text { leg }\end{array}$ & & & & & & & & $\begin{array}{l}\text { red (light } \\
\text { red } \\
\text { layers) }\end{array}$ & active \\
\hline 45 & ASP21 & Loomweight & & & & & & & & red & active \\
\hline 46 & ASP41 & $\begin{array}{l}\text { Cooking pot } \\
\text { leg }\end{array}$ & & & & & & & & red & $\begin{array}{l}\text { active- } \\
\text { slightly }\end{array}$ \\
\hline 47 & ASP48 & $\begin{array}{l}\text { Cooking pot } \\
\text { leg }\end{array}$ & & & & & & & & red & active \\
\hline 48 & ASP185 & $\begin{array}{l}\text { Cooking pot } \\
\text { leg }\end{array}$ & & & & & & & & red & active \\
\hline
\end{tabular}




\begin{tabular}{|l|l|l|l|l|l|l|l|l|l|l|l|}
\hline 49 & ASP187 & $\begin{array}{l}\text { Cooking pot } \\
\text { leg }\end{array}$ & & & & & & & & & \\
\end{tabular}

\subsubsection{SATd (FIG.8)}

This subgroup is probably identical to Kytheran Sand tempered (Kiriatzi 2003, 125; Broodbank and Kiriatzi 2007, 253-4, fig.3d-e) and should be considered as import from there. It differs from the other sand tempered fabrics with regard to frequency (higher), size (smaller grains) and sorting of inclusions (well-sorted), also presenting a more variable composition (frequent chert rock fragments, common to few mudstone, limestone and sandstone fragments).

\begin{tabular}{|c|c|c|c|c|c|c|c|c|c|c|c|}
\hline $\begin{array}{l}\text { Cat. } \\
\text { No. }\end{array}$ & Sample & $\begin{array}{c}\text { Vessel } \\
\text { Part/Shape }\end{array}$ & 党 & $\hat{2}$ & 实 & : & $\frac{\pi}{\pi}$ & & 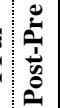 & $\begin{array}{l}\text { Refiring } \\
\text { colour }\end{array}$ & $\begin{array}{l}\text { Optical } \\
\text { Activity }\end{array}$ \\
\hline 50 & ASP177 & $\begin{array}{l}\text { Horizontal } \\
\text { handle }\end{array}$ & & & & & & & & light red & inactive \\
\hline 51 & ASP178 & $\begin{array}{l}\text { Horizontal } \\
\text { handle }\end{array}$ & & & & & & & & $\begin{array}{l}\text { light red } \\
\text { (lime } \\
\text { popping/ } \\
\text { crumbled) }\end{array}$ & slightly \\
\hline 52 & ASP179 & $\begin{array}{l}\text { Horizontal } \\
\text { handle }\end{array}$ & & & & & & & & red & $\begin{array}{l}\text { slightly- } \\
\text { inactive }\end{array}$ \\
\hline 53 & ASP43 & Body sherd & & & & & & & & $\begin{array}{l}\text { very pale } \\
\text { brown }\end{array}$ & inactive \\
\hline 54 & ASP44 & $\begin{array}{l}\text { Body sherd } \\
\text { and lug }\end{array}$ & & & & & & & & $\begin{array}{l}\text { reddish } \\
\text { yellow } \\
\text { (lug } \\
\text { refires } \\
\text { red) } \\
\end{array}$ & inactive \\
\hline 55 & ASP22 & Rim (open) & & & & & & & & $\begin{array}{l}\text { pink } \\
\text { (lime } \\
\text { popping/p } \\
\text { ieces) }\end{array}$ & slightly \\
\hline 56 & ASP196 & Pithos base & & & & & & & & $\begin{array}{l}\text { pink } \\
\text { (lime } \\
\text { popping/n } \\
\text { ot } \\
\text { extensive) }\end{array}$ & slightly \\
\hline
\end{tabular}




\subsubsection{SAT loner (FIG.8)}

57 differs significantly from the other samples, being tempered with chert and sandstone fragments, also containing weathered serpentinite fragments and a tuff fragment.

\begin{tabular}{|c|c|c|c|c|c|c|c|c|}
\hline $\begin{array}{l}\text { Cat. } \\
\text { No. }\end{array}$ & Sample & $\begin{array}{c}\text { Vessel } \\
\text { Part/Shape }\end{array}$ & $\begin{array}{l}\mathrm{z} \\
\mathrm{z} \\
\mathrm{z}\end{array}$ & $\frac{\pi}{4}$ & & & $\begin{array}{l}\text { Refiring } \\
\text { colour }\end{array}$ & $\begin{array}{l}\text { Optical } \\
\text { Activity }\end{array}$ \\
\hline 57 & ASP45 & $\begin{array}{l}\text { Jar rim and } \\
\text { handle }\end{array}$ & & & & & red & $\begin{array}{l}\text { moderately } \\
\text {-slightly }\end{array}$ \\
\hline
\end{tabular}

\subsection{GROG TEMPERED FABRICS (GRO, and subgroups GROa, b, and c)}

Grog tempered fabrics comprise about $17 \%$ of the overall prehistoric assemblage and are particularly popular in the FN-EB1 and EB2 phases, though they certainly continue throughout the later phases as well. For FN-EB1, the emphasis on grog tempering corresponds with the preferred pottery production recipes in neighbouring regions such as Kythera and western Crete (e.g. Kiriatzi 2003, 125, 'chert fabric'; Broodbank and Kiriatzi 2007, 248, fig.3b; Moody et al 2000, 'Hearth Classic'; Nodarou 2003, Day et al 2005). Nevertheless, of note is the unusual persistence of grog tempering into later prehistoric periods on Antikythera: this pattern contrasts with the one found on both Crete and Kythera and we return to discussing the possible reasons for it in the final section below. Grog-tempered fabrics cover an equally wide range of shapes as the Sand-tempered ones, including vessels of various sizes and functions.

Macroscopic fabric analysis distinguished one certain grog-tempered fabric and another that could possibly contain grog along with frequent chert fragments (see nos. 4 and 5 in the list of macroscopic groups above). Petrographic analysis verified the grog content for both macroscopic categories, which proved to be rather similar and these two were therefore incorporated into one petrographic fabric group (GRO).

The overall GRO fabric group is medium-coarse, characterized by poorly sorted subangular to sub-rounded inclusions, bimodal grain size distribution, with maximum grain size $4 \mathrm{~mm}$ (mode size $0.5-1 \mathrm{~mm}$ ) and $5-10 \%$ voids. The fine fraction consists predominantly of silicate inclusions (quartz/alkali feldspars, few chert fragments) in all samples, while the coarse fraction contains predominant to few grog fragments, frequent to absent chert rock fragments (deriving mainly from primary formations), common to few quartz, and very few alkali feldspars and quartzite rock fragments.

Three main subgroups can be distinguished on the basis of frequency and grain size of the fine fraction inclusions, groundmass optical activity, and types of inclusions. Provenance of all subgroups cannot be further discussed with present data, although features similar to Kytheran or West Cretan samples do occur sporadically.

All samples of this fabric group are tempered with grog (i.e. crushed ceramics), itself deriving from a variety of fabrics. It has to be noted that certain grog types are 
common between some or all samples. Clay mixing is not as easily discerned as in SAT fabric, but it is observed in some samples (as streaks of different colour and/or texture). The general picture of this fabric is that of the application of a standardised technique (grog tempering) in a variety of claybases and under variable firing conditions, similarly to the Sand-tempered fabric

\subsubsection{GROa (FIGS.9-10)}

This is a medium to coarse fabric, containing frequent to common chert fragments, while grog frequency is variant The fine fraction contains very fine sand up to medium sand size inclusions in an open to single-spaced porphyric related distribution. The samples are optically slightly active to inactive. Rather characteristic of this fabric is the existence of small circular Tcfs in the groundmass, dark orange in PPL and isotropic in XP (which could be marl or a post-depositional effect). The fabric is rather homogeneous in terms of composition and (bimodal) grain size distribution, but extensive variability is observed in the refiring colour, suggesting clay mixing or the use of different clay sources (it has to be noted that $\mathbf{7 1}$ and $\mathbf{7 2}$ have a rather calcareous clay base). The optical activity of the groundmass (inactive) suggests original firing temperatures exceeding $800-850^{\circ} \mathrm{C}$. The firing atmosphere was oxidising to oxidising/reducing, while the common presence of vessels with a grey core possibly implies rather short duration of firing.

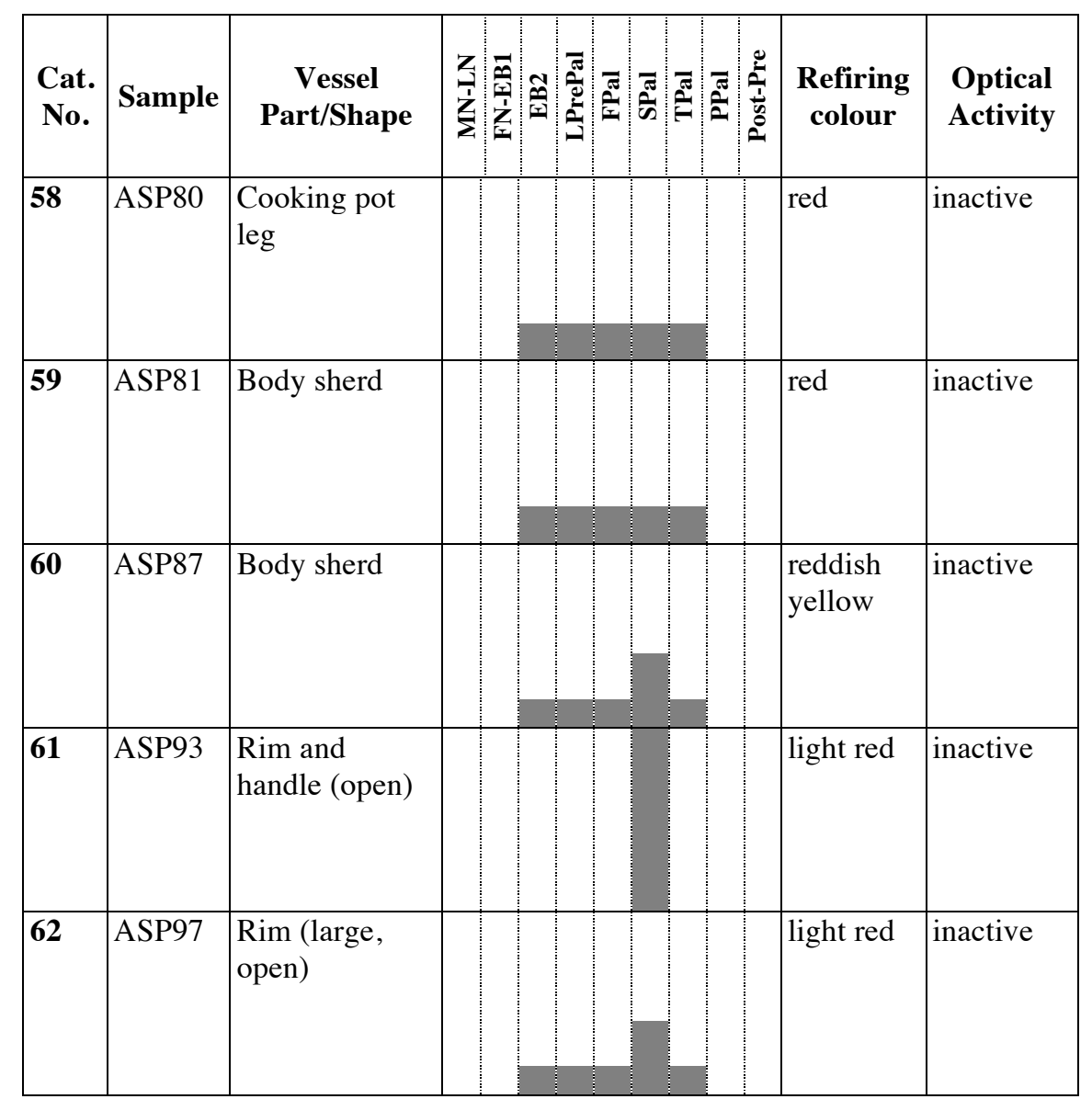




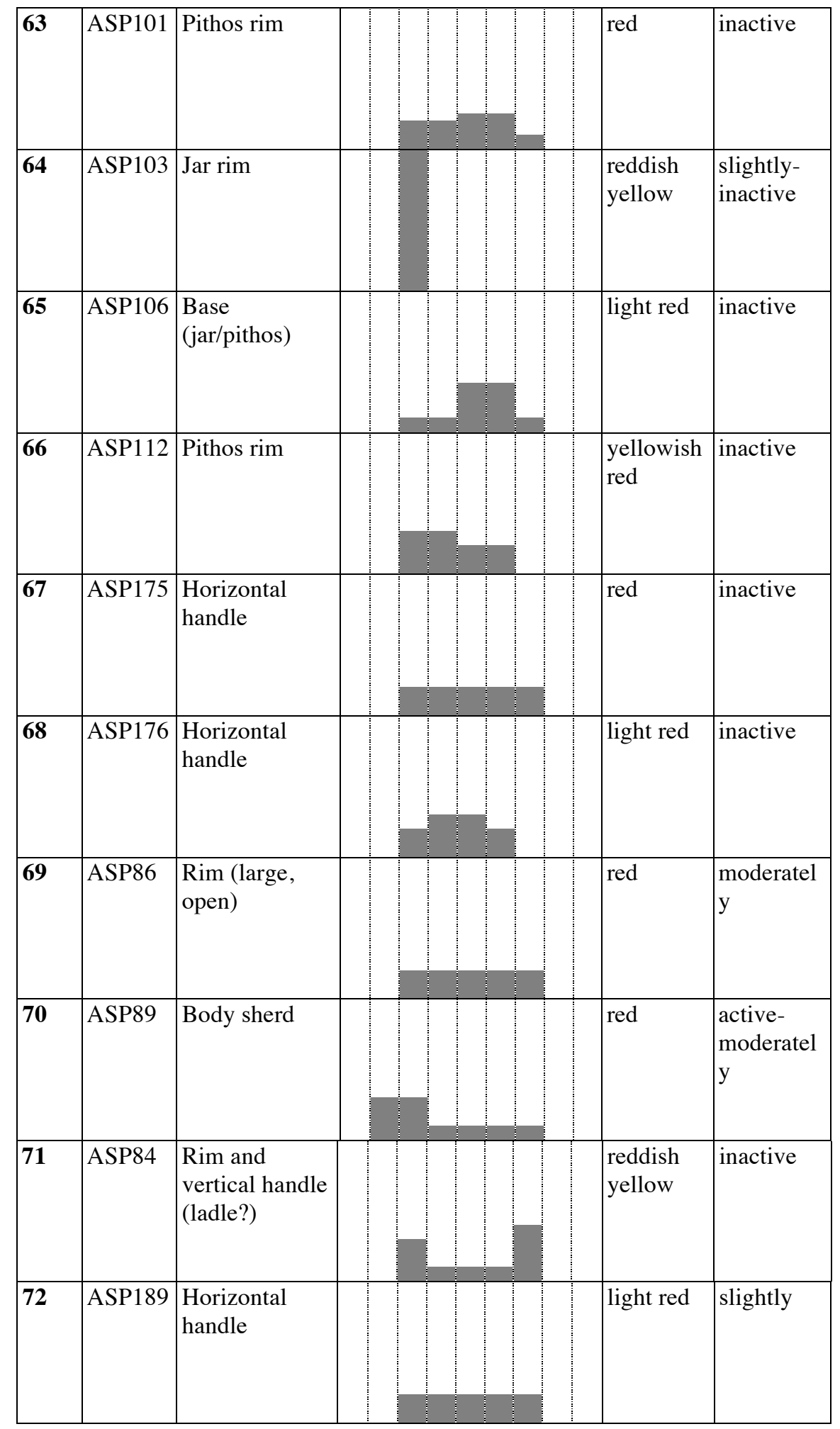

\subsubsection{GROb (FIG.11)}

This is a medium-coarse fabric, containing mainly grog, along with very dark red clay pellets. Voids are less frequent, inclusion frequency is lower and inclusion size is smaller in comparison to GROa. The fine fraction contains silt to fine sand size 
inclusions in a single to double-spaced porphyric related distribution and the samples are optically active to moderately active. The fabric is rather homogeneous in terms of composition and (bimodal) grain size distribution, with insignificant variability displayed in the refiring colour (all samples refired red, pointing to the use of similar type of clay). The optical activity of the groundmass (active to moderately active) suggests original firing temperatures below/around $800^{\circ} \mathrm{C}$, while the firing atmosphere was oxidising to oxidising/reducing.

\begin{tabular}{|c|c|c|c|c|c|c|c|c|}
\hline $\begin{array}{l}\text { Cat. } \\
\text { No. }\end{array}$ & Sample & $\begin{array}{c}\text { Vessel } \\
\text { Part/Shape }\end{array}$ & 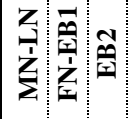 &  & $\stackrel{\pi}{\sigma}$ & 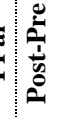 & $\begin{array}{c}\text { Refiring } \\
\text { colour }\end{array}$ & $\begin{array}{l}\text { Optical } \\
\text { Activity }\end{array}$ \\
\hline 73 & ASP72 & Body sherd & & & & & red & $\begin{array}{l}\text { active- } \\
\text { moderately }\end{array}$ \\
\hline 74 & ASP73 & Body sherd & & & & & red & $\begin{array}{l}\text { active- } \\
\text { slightly }\end{array}$ \\
\hline 75 & ASP74 & Body sherd & & & & & red & $\begin{array}{l}\text { active- } \\
\text { moderately }\end{array}$ \\
\hline 76 & ASP76 & Handle & & & & & red & active \\
\hline 77 & ASP77 & $\begin{array}{l}\text { Sauceboat (full } \\
\text { profile) }\end{array}$ & & & & & red & active \\
\hline 78 & ASP82 & Body sherd & & & & & dark red & active \\
\hline 79 & ASP85 & Rim? & & & & & red & active \\
\hline 80 & ASP102 & $\begin{array}{l}\text { Horizontal } \\
\text { handle }\end{array}$ & & & & & red & $\begin{array}{l}\text { active- } \\
\text { slightly }\end{array}$ \\
\hline 81 & ASP105 & Vertical handle & & & & & light red & slightly \\
\hline
\end{tabular}




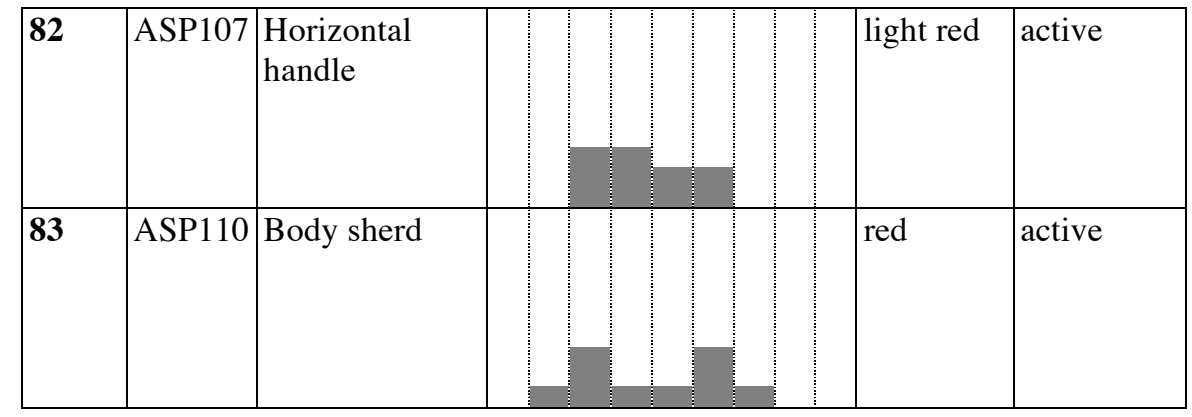

\subsubsection{GROc (FIG.12)}

This is a coarse fabric, containing almost only grog fragments. The fine fraction is characterised by silt size inclusions and the groundmass of all samples is optically inactive. The fabric is rather homogeneous in terms of composition and (bimodal) grain size distribution, with some variability displayed in the refiring colour (more than half of the samples refired red and the remaining half light red, pointing either to the use of different types of clay or to clay mix). The optical activity of the groundmass (inactive) suggests original firing temperatures exceeding $800^{\circ} \mathrm{C}$, while the prevailing firing atmosphere was reducing.

\begin{tabular}{|c|c|c|c|c|c|c|c|c|c|}
\hline $\begin{array}{l}\text { Cat. } \\
\text { No. }\end{array}$ & Sample & $\begin{array}{c}\text { Vessel } \\
\text { Part/Shape }\end{array}$ & 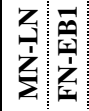 & & & ๘) & ؛ั๋ & $\begin{array}{c}\text { Refiring } \\
\text { colour }\end{array}$ & $\begin{array}{l}\text { Optical } \\
\text { Activity }\end{array}$ \\
\hline 84 & ASP100 & Jar rim & & & & & & light red & inactive \\
\hline 85 & ASP113 & Jar rim & & & & & & red & inactive \\
\hline 86 & ASP118 & Base & & & & & & red & inactive \\
\hline 87 & ASP94 & Jar rim & & & & & & light red & inactive \\
\hline 88 & ASP116 & $\begin{array}{l}\text { Horizontal } \\
\text { handle }\end{array}$ & & & & & & red & inactive \\
\hline
\end{tabular}




\subsubsection{GRO loners (FIG.13)}

The remaining GRO samples present compositional or textural differences that keep them apart from the main GRO subgroups. 89 bears a fine-grained groundmass, is also siltstone-tempered and contains few white mica aggregates. 90 contains rare siltstone and serpentinite fragments, while 91 bears a very fine micaceous groundmass. 92 contains also biotite schist rock fragments; 93 bears a dark red, possibly iron-rich, matrix, whereas the matrix of 94 is exceptionally fine. Finally, 95 also contains limestone fragments.

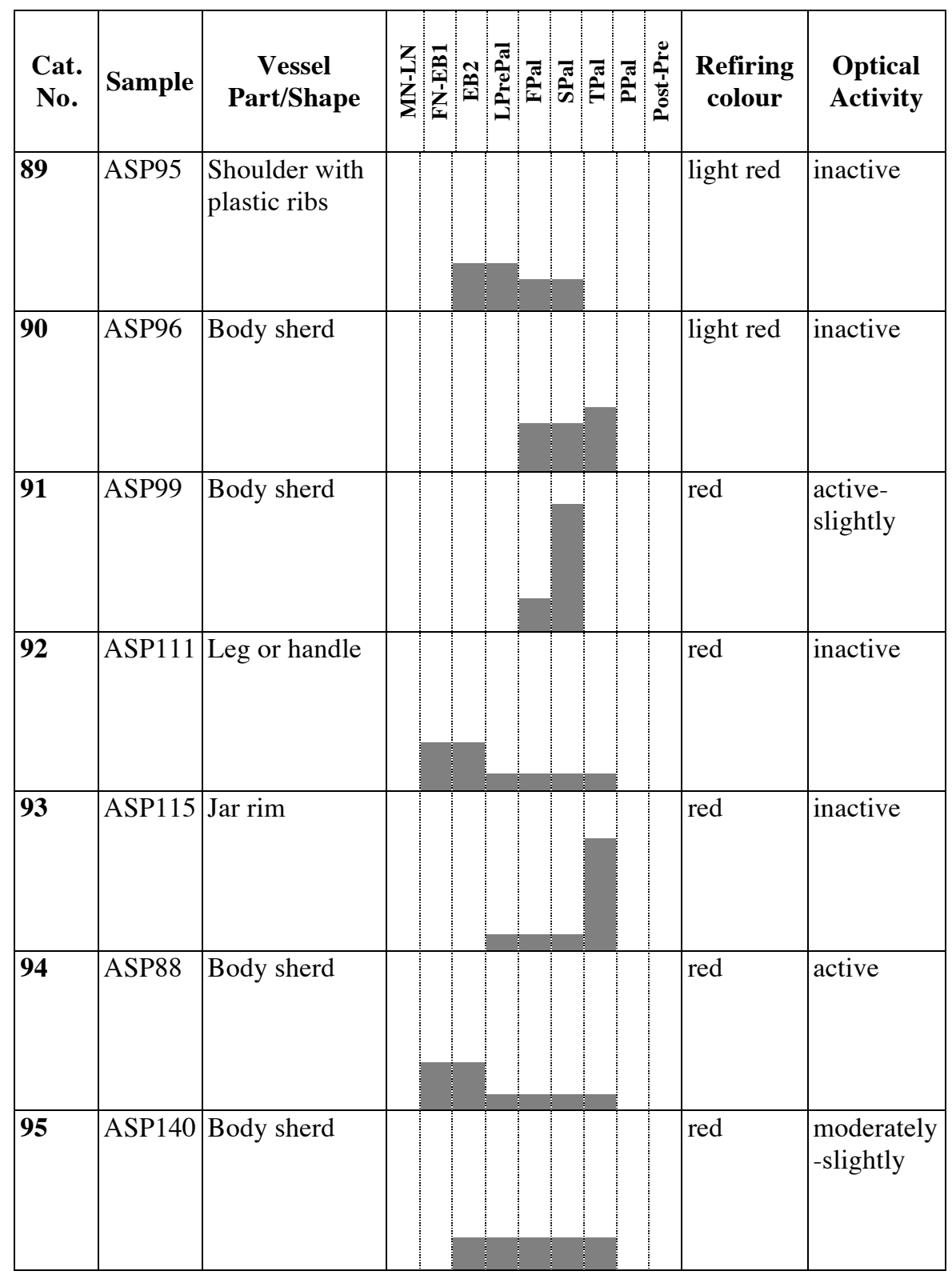

2.3 MUDSTONE TEMPERED FABRICS (MUT, and subgroups MUTa, b, and c)

Mudstone tempered fabrics comprise just over $10 \%$ of the overall prehistoric assemblage found on Antikythera. Mudstone tempering seems to begin at least as 
early as EMIIA in western Crete (Nodarou pers comm.), but is found more commonly in First to Third Palace sites on Kythera and in western Crete (Moody 1985, 56; Moody et al 2000, 'Mixed Red Chip'; Kiriatzi 2003, 125). It is less common but present on the rest of Crete (e.g. Mook 2005, 172, type XIII). On Antikythera, the stylistic and contextual evidence suggest the uptake and use of mudtsone tempered fabrics roughly in step with these regions, and closely matching previous observations about immediately neighbouring areas such as Gramvousa and Chysoskalitissa (e.g. Moody 1985, 56). More precisely, while there are possible early examples of mudstone tempering found in association with EB2-late Prepalatial scatters on Antikythera, we see clear evidence for such fabrics during the palatial periods, and they are particularly common in the Second and Third Palace periods. With regard to shapes, Mudstone tempered fabrics are associated mostly with medium to large size storage vessels and medium size cooking pots (i.e. with the same narrow range of vessel types observed also in the Micaceous fabric discussed below).

Macroscopic fabric analysis distinguished two mudstone-tempered fabrics, and one of which seemed to be from Kythera. The latter was also identified as a separate group (MUTc) by petrographic analysis, and the remaining mudstone samples were divided into two further sub-groups.

The overall MUT fabric group is coarse, characterised by poorly sorted angular to sub-rounded inclusions, bimodal grain size distribution, with maximum grain size $5.6 \mathrm{~mm}$ (mode size $0.5-1 \mathrm{~mm}$ ) and $5-10 \%$ voids. The fine fraction consists predominantly of silicate inclusions (quartz/alkali feldspars, few chert fragments), while the coarse fraction contains predominant to dominant mudstone rock fragments, few quartz, chert fragments and alkali feldspars, and few to absent siltstone rock fragments. Three sub-groups were distinguished on the basis of frequency and grain size of the fine fraction inclusions, and types of inclusions. The provenance of MUTa and MUTb cannot be safely documented with present data, although a west Cretan origin appears quite possible. Sub-group MUTc bears a strong resemblance to Kytheran Mudstone tempered fabric, so a Kytheran origin could be proposed for this sub-group.

All samples of this overall fabric group are consistently tempered with mudstone fragments, while clay mixing is rather infrequently observed. The general picture of this fabric, similarly to the previous two already discussed, is that of the application of a standardised technique (mudstone tempering) in a variety of claybases and under more or less the same firing conditions (high temperatures).

\subsubsection{MUTa (FIG.14)}

This is a coarse fabric, containing angular to sub-angular mudstone fragments and few chert (with instances of radiolarian chert in one or two samples). The fine fraction contains fine sand size inclusions in a single-spaced porphyric related distribution. The groundmass of MUTa resembles that of SATa and GROa, mainly in terms of the silicate inclusions and their grain size, since the unidentified Tcfs observed in GROa are present, but considerably fewer. The fabric is homogeneous in terms of composition and (bimodal) grain size distribution, although the refiring colour is not identical in all samples (red tones prevail, pointing either to clay mixing or to the use of different types of clay). The groundmass is optically inactive suggesting original firing temperatures exceeding $800^{\circ} \mathrm{C}$. The firing atmosphere was oxidising to 
oxidising/reducing, and the common presence of a grey core possibly implies a rather short duration of firing.

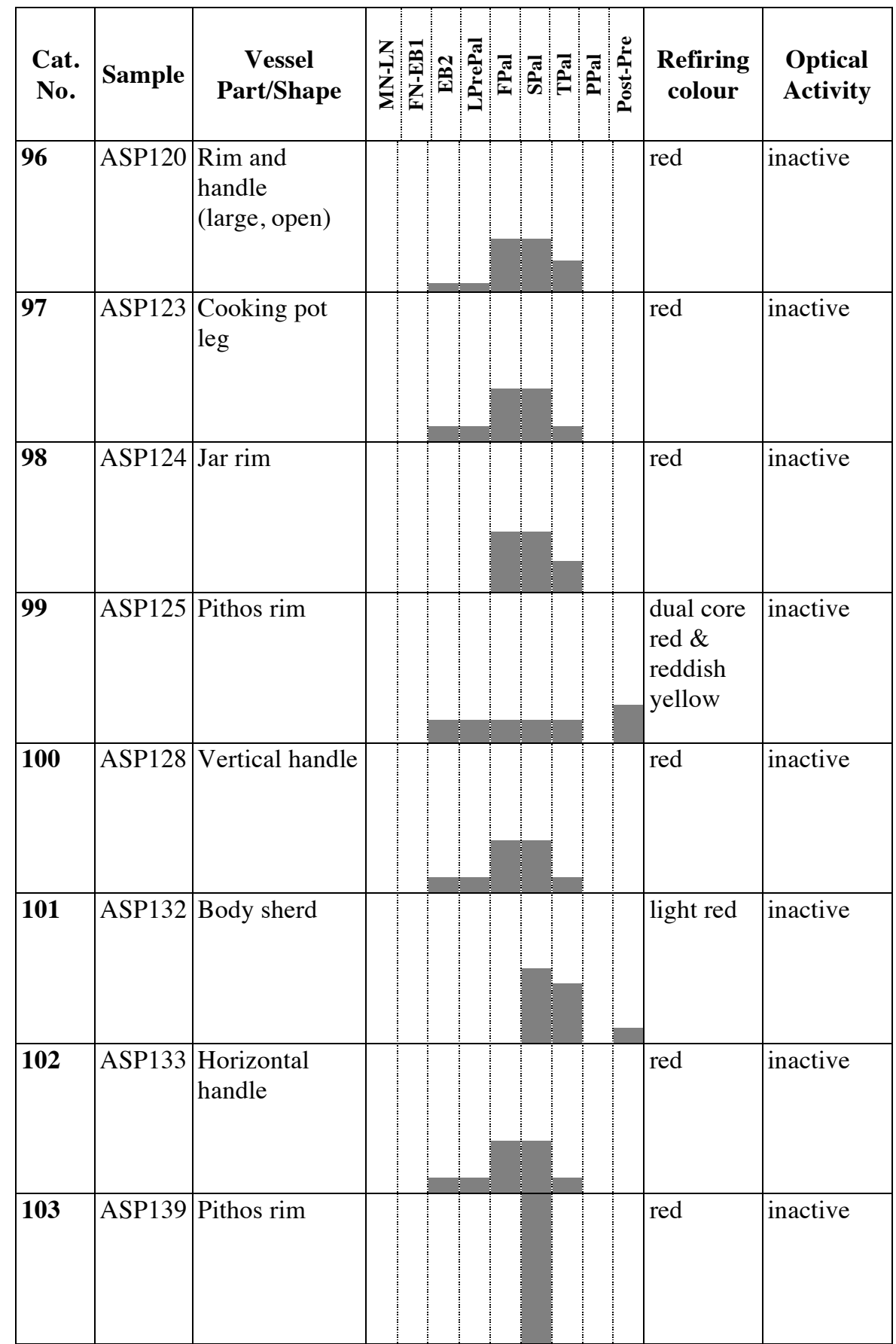

\subsubsection{MUTb (FIG.15)}

This is a coarse fabric, containing siltstone fragments, along with mudstone and chert fragments, both frequently radiolarian (104 contains also a possible acid tuff). The fine fraction is characterised by silt size inclusions in a single to double-spaced porphyric related distribution. The fabric is homogeneous in terms of composition, (bimodal) grain size distribution, and refiring (red). The optical activity of the groundmass (moderately active to inactive) suggests original firing temperatures around / over $800^{\circ} \mathrm{C}$, while the firing atmosphere was oxidising to variable. 


\begin{tabular}{|c|c|c|c|c|c|c|c|c|}
\hline $\begin{array}{l}\text { Cat. } \\
\text { No. }\end{array}$ & Sample & $\begin{array}{c}\text { Vessel } \\
\text { Part/Shape }\end{array}$ & 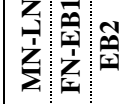 & 通 & बँ & : & $\begin{array}{l}\text { Refiring } \\
\text { colour }\end{array}$ & $\begin{array}{l}\text { Optical } \\
\text { Activity }\end{array}$ \\
\hline 104 & ASP126 & Jar base & & & & & red & $\begin{array}{l}\text { moderately } \\
\text {-slightly }\end{array}$ \\
\hline 105 & ASP129 & Body sherd & & & & & red & inactive \\
\hline 106 & ASP130 & Body sherd & & & & & red & $\begin{array}{l}\text { active- } \\
\text { slightly }\end{array}$ \\
\hline 107 & ASP138 & $\begin{array}{l}\text { Cooking pot } \\
\text { leg }\end{array}$ & & & & & red & inactive \\
\hline
\end{tabular}

\subsubsection{MUTc (FIG.16)}

This is a coarse fabric, consisting predominantly of mudstone fragments, along with few chert fragments and carbonate inclusions. The fine fraction contains almost no distinguishable inclusions. This subgroup is very similar if not identical to Kytheran Mudstone tempered (Kiriatzi 2003, 125) and is considered to be an import from Kythera. The fabric is homogeneous in terms of composition and (bimodal) grain size distribution but presents variability in refiring colour (from red to very pale brown and pink), pointing to clay mixing or use of different raw materials sources. The optical activity of the groundmass (slightly active to inactive) suggests original firing temperatures exceeding $800^{\circ} \mathrm{C}$, while the firing atmosphere was oxidising to variable.

\begin{tabular}{|c|c|c|c|c|c|c|c|c|c|c|c|}
\hline $\begin{array}{l}\text { Cat. } \\
\text { No. }\end{array}$ & Sample & $\begin{array}{c}\text { Vessel } \\
\text { Part/Shape }\end{array}$ & 亮: & 公: & s: & $\frac{\sqrt{5}}{x}$ & $\tilde{\pi}^{\pi}$ & & 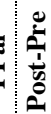 & $\begin{array}{l}\text { Refiring } \\
\text { colour }\end{array}$ & $\begin{array}{l}\text { Optical } \\
\text { Activity }\end{array}$ \\
\hline 108 & ASP141 & Body sherd & & & & & & & & pink & slightly \\
\hline 109 & ASP142 & $\begin{array}{l}\text { Horizontal } \\
\text { handle }\end{array}$ & & & & & & & & $\begin{array}{l}\text { very pale } \\
\text { brown }\end{array}$ & inactive \\
\hline
\end{tabular}




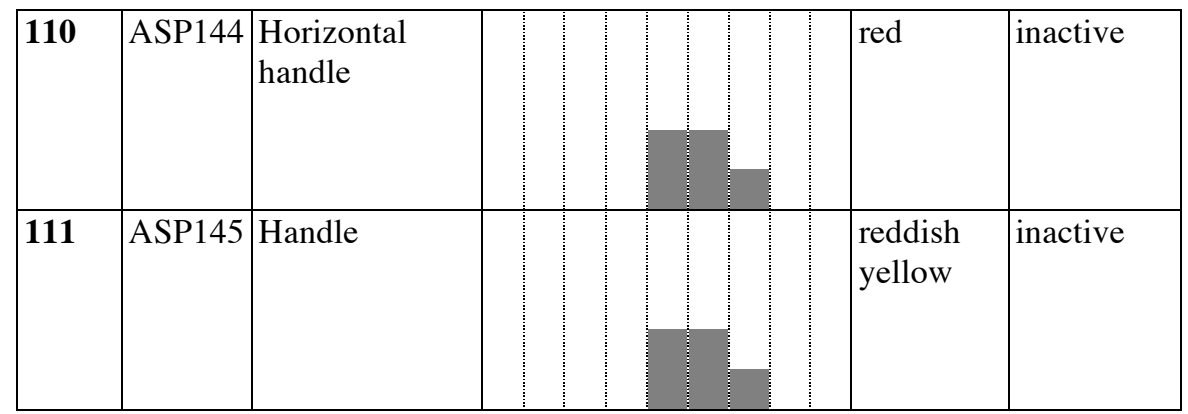

\subsubsection{MUT loners (FIG.16)}

112, 113, 114 are connected to MUT fabric, but differ in certain aspects: 111 is very high fired / vitrified, making further microscopic examination difficult; 113 is characterised by a rather micaceous groundmass; and in 114, the fine fraction contains up to medium sand size silicate inclusions in a single-spaced porphyric related distribution.

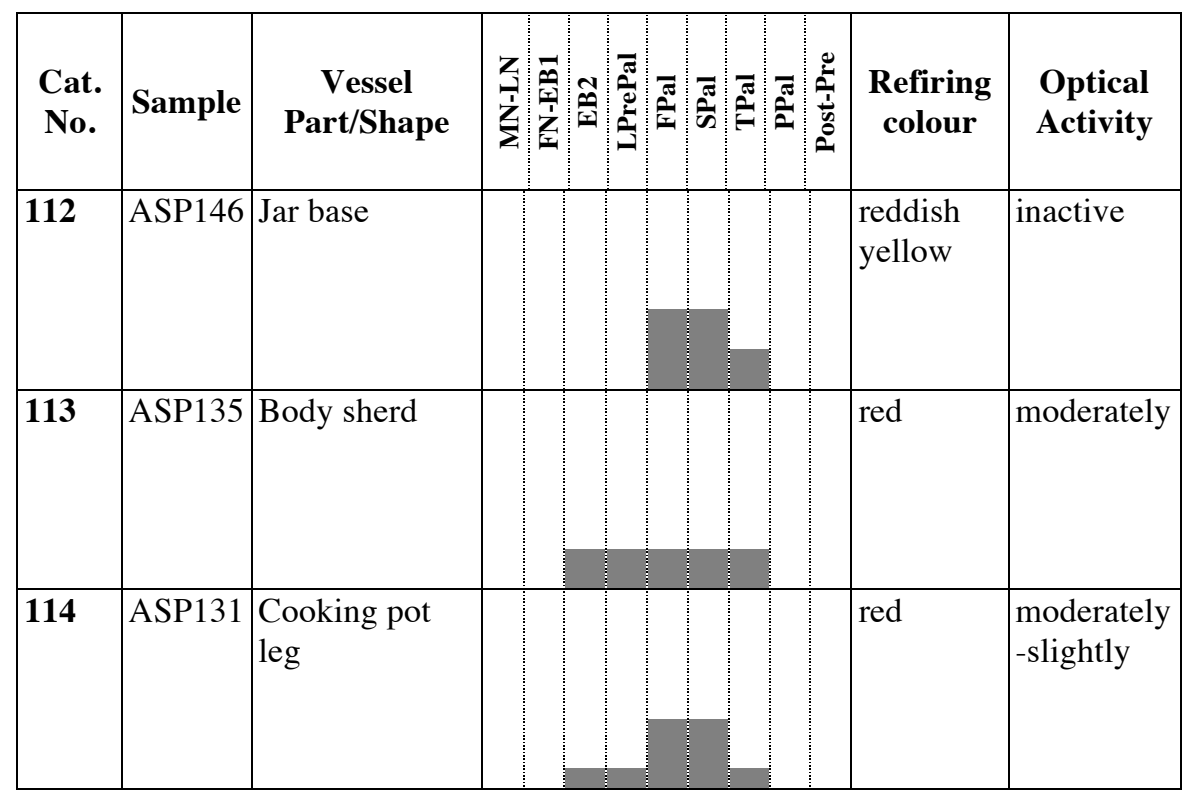

\subsection{CALCITE TEMPERED FABRICS (CAL, and subgroups CALa, b, and c)}

Calcite tempered fabrics comprise just over $6 \%$ of the overall prehistoric assemblage from Antikythera. Such fabrics are found widely in western Crete (Moody 1985, 56; Moody et al 2000, 'Calcareous Fabrics'; Moody et al 2003, 64-5) and elsewhere (Barnard 2003, 10, type 13; Day et al 2005, 18-19, group 3; Moody 2005, 153-4, 'Calcareous/Calcite group A and B'; Mook 2005, 171, fabric IX). They tend to be of FN to First Palace date but seem to be particularly common in EMI-IIA and MMI-II. The evidence from Antikythera seems broadly in step with these date ranges. With regard to shapes, Calcite tempered fabrics on Antikythera seem to cover a very limited range of shapes, mostly cooking pots of medium size.

Macroscopic examination observations and petrographic analysis results coincided in the identification of this fabric and its members, with those originally identified as 
'dense and red firing' falling into petrographic sub-group CALa and those identified as 'buff-tan firing with frequent poorly sorted sub-angular limestone' (see no. 3 in the list of macroscopic groups above) being petrographically divided into CALb and CALc.

The overall CAL fabric group is coarse, characterised by poorly sorted angular to subrounded inclusions, bimodal grain size distribution, with maximum grain size $4.6 \mathrm{~mm}$ (mode size $0.3-0.7 \mathrm{~mm}$ ) and $5-10 \%$ voids. The fine fraction consists predominantly of silicate inclusions (quartz/alkali feldspars, few chert fragments), while the coarse fraction contains predominant limestone fragments, few to very few quartz and alkali feldspars, and very few to absent chert fragments. With the present data, it is impossible to comment on provenance of any of the subgroups; local origin for some of the samples should not be immediately ruled out as a possibility, though.

Apart from tempering with limestone (crushing is not improbable), clay mixing seems to be practiced as well, since a few clay pellets and streaks of different colour and/or texture are observed. Again, the general picture of this fabric is that of the application of a standardised technique (tempering) in a variety of claybases. Also, this fabric seems to be consistently fired at lower temperatures than the other recognised fabric groups; this could point to a conscious choice on the potters' part, in order to avoid lime-popping of coarse calcite inclusions and subsequent collapsing of the vessel, and reveals experience in controlling raw materials (Rice 1987, 97-98).

\subsubsection{CALa (FIG.17)}

This is a coarse fabric, containing angular crystalline calcite and some chert fragments (except 115 and 121), few Tcfs/clay pellets and micrite lumps in a groundmass characterised by silt to fine sand-sized inclusions in an open to single-spaced porphyric related distribution. The fabric is homogeneous in terms of composition, (bimodal) grain size distribution, and refiring colour (red). Almost all refired pieces crumbled after some weeks, due to the transformation of coarse calcite inclusions into lime and their concomitant expansion in volume. Therefore, the original firing temperature should be below $800^{\circ} \mathrm{C}$, confirmed also by the groundmass optical activity. The firing atmosphere was oxidising to oxidising/reducing, while the common presence of grey cores possibly reflects a quick rise of temperature and short firing duration.

\begin{tabular}{|c|c|c|c|c|c|c|c|c|}
\hline $\begin{array}{l}\text { Cat. } \\
\text { No. }\end{array}$ & Sample & $\begin{array}{c}\text { Vessel } \\
\text { Part/Shape }\end{array}$ & 晏案 & 产产 & $\begin{array}{l}\frac{\pi}{5} \\
\frac{1}{2}\end{array}$ & $\frac{\pi}{6} \frac{\pi}{2}$ & $\begin{array}{c}\text { Refiring } \\
\text { colour }\end{array}$ & $\begin{array}{l}\text { Optical } \\
\text { Activity }\end{array}$ \\
\hline 115 & ASP49 & Rim or lid & & & & & $\begin{array}{l}\text { red (lime } \\
\text { popping/c } \\
\text { rumbled) }\end{array}$ & $\begin{array}{l}\text { active- } \\
\text { moderately }\end{array}$ \\
\hline 116 & ASP50 & $\begin{array}{l}\text { Cooking pot } \\
\text { leg }\end{array}$ & & & & & $\begin{array}{l}\text { red (lime } \\
\text { popping/c } \\
\text { rumbled) }\end{array}$ & $\begin{array}{l}\text { moderately } \\
\text {-slightly }\end{array}$ \\
\hline
\end{tabular}




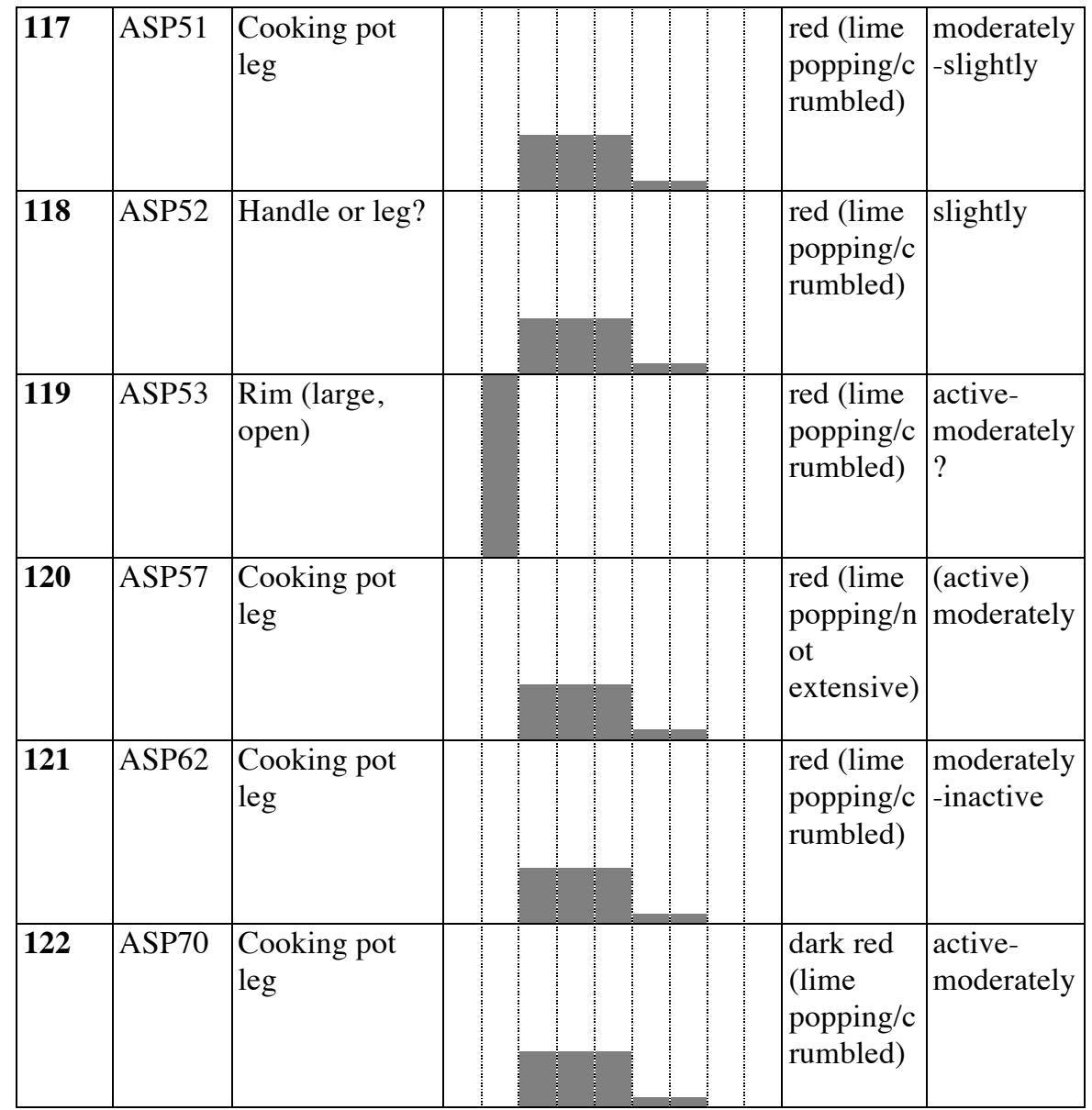

\subsubsection{CALb (FIG.17)}

This is a coarse fabric, containing limestone fragments and few clay pellets only, in a fine groundmass that vividly resembles SATc (i.e. the fine fraction contains almost no distinguishable inclusions). The fabric is homogeneous in terms of composition, (bimodal) grain size distribution, and refiring colour (red tones). Almost all refired pieces crumbled after some weeks (similarly to CALa above). The optical activity of the groundmass (active and very birefringent micromass) and the more or less unaltered calcite grains suggest original firing temperatures below $800^{\circ} \mathrm{C}$ (possibly considerably lower in comparison to the other two sub-groups), while the firing atmosphere was oxidising.

\begin{tabular}{|c|c|c|c|c|c|c|c|c|c|c|c|c|}
\hline $\begin{array}{l}\text { Cat. } \\
\text { No. }\end{array}$ & Sample & $\begin{array}{c}\text { Vessel } \\
\text { Part/Shape }\end{array}$ & 辛 & 离: & 요요 & 20 & 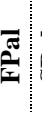 & & & $\frac{1}{1}$ & $\begin{array}{l}\text { Refiring } \\
\text { colour }\end{array}$ & $\begin{array}{l}\text { Optical } \\
\text { Activity }\end{array}$ \\
\hline 123 & ASP54 & $\begin{array}{l}\text { Cooking pot } \\
\text { leg }\end{array}$ & & & & & & & & & $\begin{array}{l}\text { red (lime } \\
\text { popping/c } \\
\text { rumbled) }\end{array}$ & active \\
\hline
\end{tabular}




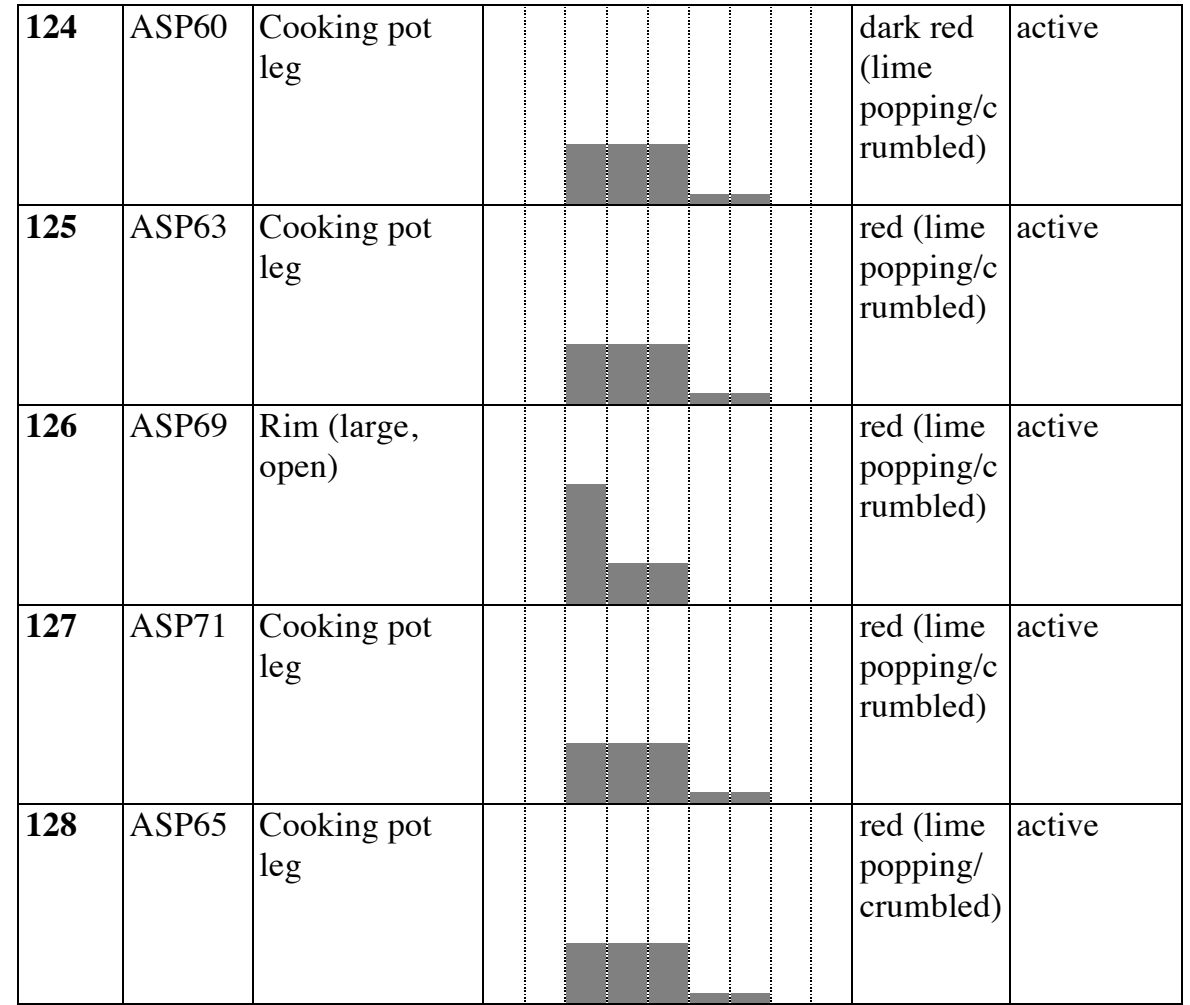

\subsubsection{CALc (FIG.18)}

This is a coarse fabric, consisting of limestone fragments and few clay pellets only. The fabric is characterised by a fine fraction containing silt-sized inclusions in an open to double-spaced porphyric related distribution and is homogeneous in terms of composition, (bimodal) grain size distribution, and refiring colour (red tones). Almost all refired pieces crumbled after some weeks, due to lime popping of coarse calcite inclusions. The optical activity of the groundmass (moderately-slightly) and the slightly altered calcite grains suggest original firing temperatures below/around $800^{\circ} \mathrm{C}$. The firing atmosphere was oxidising to oxidising/reducing, while the common presence of grey cores possibly reflects a quick rise of temperature and short firing duration.

\begin{tabular}{|c|c|c|c|c|c|c|c|c|c|c|c|c|}
\hline $\begin{array}{l}\text { Cat. } \\
\text { No. }\end{array}$ & Sample & $\begin{array}{c}\text { Vessel } \\
\text { Part/Shape }\end{array}$ & ż & 竞 & $\begin{array}{c}\alpha \\
0\end{array}$ & 犬ิ & $\frac{\pi}{5}$ & बै & $\frac{\pi}{2}$ & 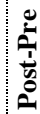 & $\begin{array}{l}\text { Refiring } \\
\text { colour }\end{array}$ & $\begin{array}{l}\text { Optical } \\
\text { Activity }\end{array}$ \\
\hline 129 & ASP55 & $\begin{array}{l}\text { Cooking pot } \\
\text { leg }\end{array}$ & & & & & & & & & $\begin{array}{l}\text { red (lime } \\
\text { popping/c } \\
\text { rumbled) }\end{array}$ & moderately \\
\hline 130 & $\begin{array}{l}\text { ASP58 } \\
\text { and } \\
\text { ASP61 }\end{array}$ & $\begin{array}{l}\text { Cooking pot } \\
\text { (handle and } \\
\text { leg) }\end{array}$ & & & & & & & & & $\begin{array}{l}\text { red (lime } \\
\text { popping/c } \\
\text { rumbled) }\end{array}$ & $\begin{array}{l}\text { active- } \\
\text { slightly }\end{array}$ \\
\hline
\end{tabular}




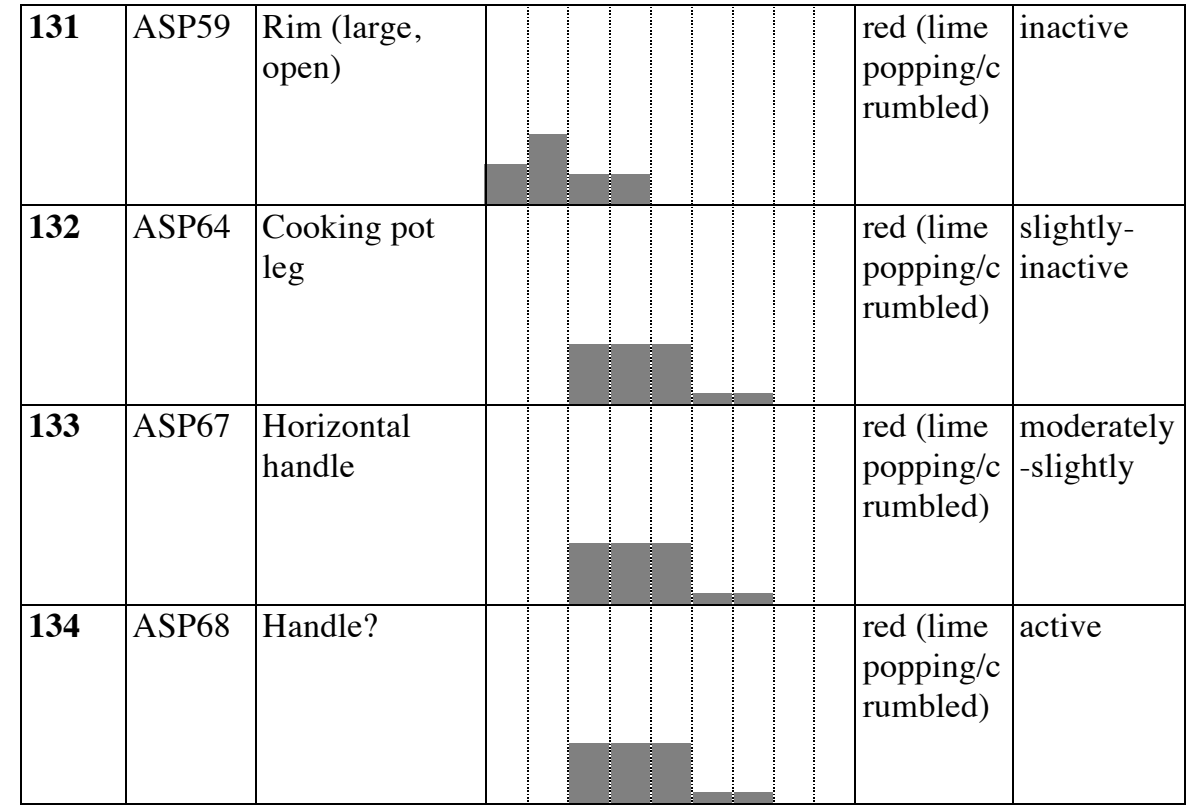

\subsubsection{CAL loner (FIG.18)}

135 differs from the other calcite-tempered samples in that it presents crystalline limestone fragments covering a range of grain size (grain-size distribution being less evidently bimodal) and contains also rare serpentinite fragments.

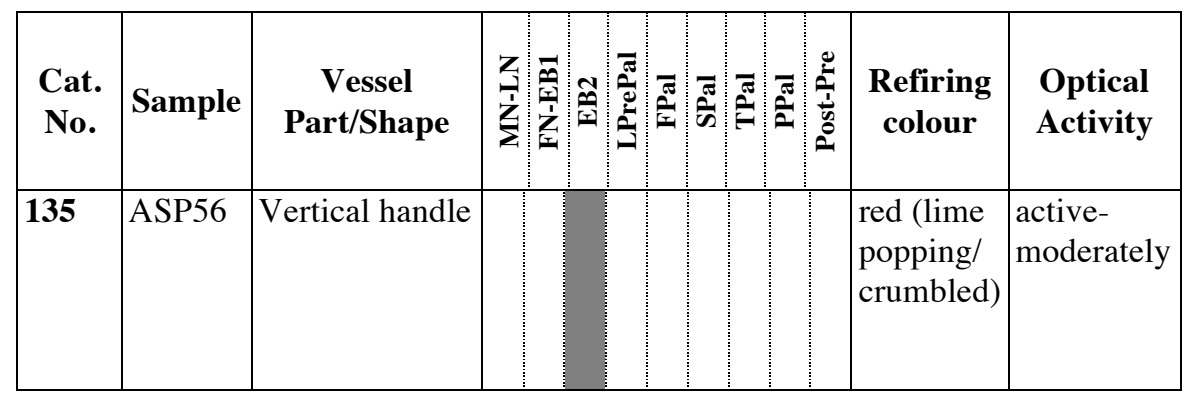

\subsection{PHYLLITE/SHALE AND QUARTZITE FABRICS (SHA, and subgroups} SHAa, b, c, d and e)

Phyllite/shale and quartzite fabrics comprise just under $3 \%$ of the overall prehistoric assemblage, but are of particular interest because they are incompatible with the local Antikytheran geology and are very likely to have come from western Crete where such fabrics are common throughout the EM-LMIII periods, and particularly during LM phases (Moody 1985, 55; Moody et al 2000, 'Phyllite Cooking'). Similar fabrics also exist EM-LM eastern Crete (e.g. Mook 2005, 71, 73, types I/IV; Barnard 2003, 5), but we suspect that analogous fabrics are also used in the Medieval period as well, so occasionally the dating of individual samples is much broader. All the subgroups discussed below are very similar to Chandler's A1 fabric (2001), and usually identical with one or two of his samples. All samples seem to be deliberately tempered, with a small portion of metamorphic rock fragments being added to a rather coarse primary sediment. Phyllite/shale and quartzite fabrics found on Antikythera cover a rather narrow range of vessels, being associated mostly with jars (wide-mouthed or necked) of medium size. 
Macroscopic examination and petrographic analysis coincided in the identification of this fabric. It is always medium-coarse to coarse, characterised by poorly sorted angular to sub-rounded inclusions, weak bimodal grain size distribution, with maximum grain size $4 \mathrm{~mm}$ (mode size $0.5-1 \mathrm{~mm}$ ) and around $5 \%$ voids. The claybase contains fine to medium sand size grains, mainly silicates, while the fabric is characterized by frequent to common phyllite/shale and quartzite fragments, common to few mica schist rock fragments and quartz, and few to very few alkali feldspars, black opaques and micas (in 136, 137, 138, 139, 142, 148 also rare grains with blue / blue-green pleochroism that are difficult to identify due to small grain size, but could be amphiboles).

\subsubsection{SHAa (FIG.19)}

This is a coarse fabric, containing phyllite/shale, quartz sand, quartzite and mica schist, in a fine to very fine sand size groundmass with blue grains. The fabric is homogeneous in terms of composition, grain size distribution, and refiring colour (red). The optical activity of the groundmass (active-slightly) suggests original firing temperatures around $800^{\circ} \mathrm{C}$, while the firing atmosphere was oxidising to oxidising/reducing.

\begin{tabular}{|c|c|c|c|c|c|c|c|c|}
\hline $\begin{array}{l}\text { Cat. } \\
\text { No. }\end{array}$ & Sample & $\begin{array}{c}\text { Vessel } \\
\text { Part/Shape }\end{array}$ & $\begin{array}{l}\bar{z} \\
\vec{z} \\
\dot{z} \\
\dot{z}\end{array}$ & & 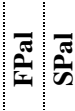 & ลิ) & $\begin{array}{c}\text { Refiring } \\
\text { colour }\end{array}$ & $\begin{array}{l}\text { Optical } \\
\text { Activity }\end{array}$ \\
\hline 136 & ASP155 & Leg? & & & & & red & $\begin{array}{l}\text { moderately } \\
\text {-slightly }\end{array}$ \\
\hline 137 & ASP158 & Handle or leg & & & & & red & $\begin{array}{l}\text { active- } \\
\text { moderately }\end{array}$ \\
\hline
\end{tabular}

\subsubsection{SHAb (FIG.19)}

This is a coarse fabric, containing phyllite/shale quartz sand, quartzite and mica schist, well sorted, with blue grains (but not in 140) in the groundmass. The fabric is homogeneous in terms of composition, grain size distribution, and refiring colour (red tones). The optical activity of the groundmass (active-moderately) suggests original firing temperatures below/around $800^{\circ} \mathrm{C}$, while the firing atmosphere was oxidising to oxidising/reducing.

\begin{tabular}{|c|c|c|c|c|c|c|c|c|c|}
\hline $\begin{array}{l}\text { Cat. } \\
\text { No. }\end{array}$ & Sample & $\begin{array}{c}\text { Vessel } \\
\text { Part/Shape }\end{array}$ & $\begin{array}{ll}z & 0 \\
\dot{z} & 0 \\
\dot{z}\end{array}$ & t) & ๘) & $\frac{\pi}{2}$ & 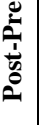 & $\begin{array}{l}\text { Refiring } \\
\text { colour }\end{array}$ & $\begin{array}{r}\text { Optical } \\
\text { Activity }\end{array}$ \\
\hline
\end{tabular}




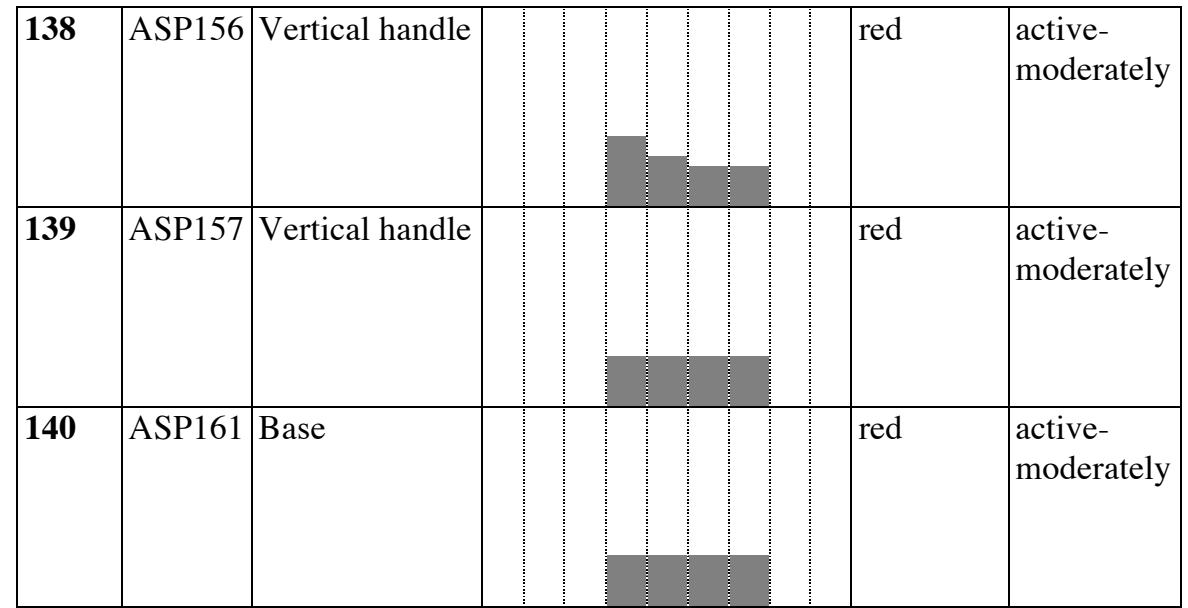

\subsubsection{SHAc (FIG.19)}

This is a coarse fabric, that bears very strong similarity to Chania GSE27 (Chandler 2001), with phyllite/shale quartz sand, quartzite and mica schist, in a fine sand size groundmass (no blue grains in 139, abundant blue grains in 140). The fabric is homogeneous in terms of composition, grain size distribution, and refiring colour (red tones). The optical activity of the groundmass (active-inactive) suggests original variable firing temperatures around $800^{\circ} \mathrm{C}$, while the firing atmosphere was oxidising to oxidising/reducing.

\begin{tabular}{|c|c|c|c|c|c|c|c|c|c|}
\hline $\begin{array}{l}\text { Cat. } \\
\text { No. }\end{array}$ & Sample & $\begin{array}{c}\text { Vessel } \\
\text { Part/Shape }\end{array}$ & 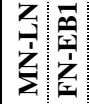 & & 눈 & & $\frac{1}{10}$ & $\begin{array}{l}\text { Refiring } \\
\text { colour }\end{array}$ & $\begin{array}{l}\text { Optical } \\
\text { Activity }\end{array}$ \\
\hline 141 & ASP152 & Body sherd & & & & & & red & inactive \\
\hline 142 & ASP159 & Handle & & & & & & red & \begin{tabular}{|l|} 
active- \\
moderately
\end{tabular} \\
\hline
\end{tabular}

\subsubsection{SHAd (FIG.19)}

This is a coarse fabric, where shale prevails in a fine sand size groundmass (as in SHAa, in 144 also carbonates, without blue grains). Resemblance to Chandler's A1 (2001) is not as strong as in the previous groups, but it is not entirely dissimilar either. The fabric is rather homogeneous in terms of composition, grain size distribution, and refiring colour (light red-pink colours, implying the use of a different clay source in comparison to the previous subgroups). The optical activity of the groundmass (inactive) suggests original firing temperatures around $800^{\circ} \mathrm{C}$, while the firing atmosphere was oxidising to oxidising/reducing. 


\begin{tabular}{|c|c|c|c|c|c|c|c|c|c|}
\hline $\begin{array}{l}\text { Cat. } \\
\text { No. }\end{array}$ & Sample & $\begin{array}{c}\text { Vessel } \\
\text { Part/Shape }\end{array}$ & 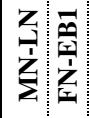 & 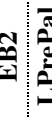 & ๘) & कึ & : & $\begin{array}{c}\text { Refiring } \\
\text { colour }\end{array}$ & $\begin{array}{l}\text { Optical } \\
\text { Activity }\end{array}$ \\
\hline 143 & ASP150 & Jar rim & & & & & & $\begin{array}{l}\text { reddish } \\
\text { yellow }\end{array}$ & inactive \\
\hline 144 & ASP153 & Vertical handle & & & & & & pink & inactive \\
\hline
\end{tabular}

\subsubsection{SHAe (FIG.20)}

This is a coarse fabric, where quartzite and quartz prevail, without blue grains in the groundmass. It bears a strong resemblance to Chania sample GSE24 and Chandler's geological sample 5 (metamorphic clay, Galatas highlands, Chandler 2001). The fabric is homogeneous in terms of composition, grain size distribution, and refiring colour (red). The optical activity of the groundmass (inactive) suggests original firing temperatures exceeding $800^{\circ} \mathrm{C}$, while the firing atmosphere was oxidising to oxidising/reducing.

\begin{tabular}{|c|c|c|c|c|c|c|c|c|}
\hline $\begin{array}{l}\text { Cat. } \\
\text { No. }\end{array}$ & Sample & $\begin{array}{c}\text { Vessel } \\
\text { Part/Shape }\end{array}$ & 辛离 & $\frac{6}{2}$ & t. & ڤิ山 & $\begin{array}{c}\text { Refiring } \\
\text { colour }\end{array}$ & $\begin{array}{l}\text { Optical } \\
\text { Activity }\end{array}$ \\
\hline 145 & ASP149 & Rim & & & & & red & inactive \\
\hline 146 & ASP160 & Vertical handle & & & & & red & inactive \\
\hline 147 & ASP172 & $\begin{array}{l}\text { Horizontal } \\
\text { handle }\end{array}$ & & & & & red & inactive \\
\hline
\end{tabular}

\subsubsection{SHA loners (FIG.20)}

Three samples differ from the other members of the fabric group in terms of grain size distribution and inclusions types. 148 presents strong bimodal grain size distribution, the fine fraction consisting of fine silt size inclusions in a double-spaced porphyric related distribution, whereas the coarse fraction consists of well sorted and rounded to subrounded quartz sand, reminding Chandler's A1 sample GSE5 (2001). 149 also presents strong bimodal grain size distribution, the fine fraction containing fine silt 
size silicate and mica inclusions in a double-spaced porphyric related distribution. 150 contains phyllite/shale fragments only, along with small grains of probably weathered serpentinite. Finally, 152 contains considerably rare micas and sphene, while the groundmass presents grey unidentified Tcfs in PPL.

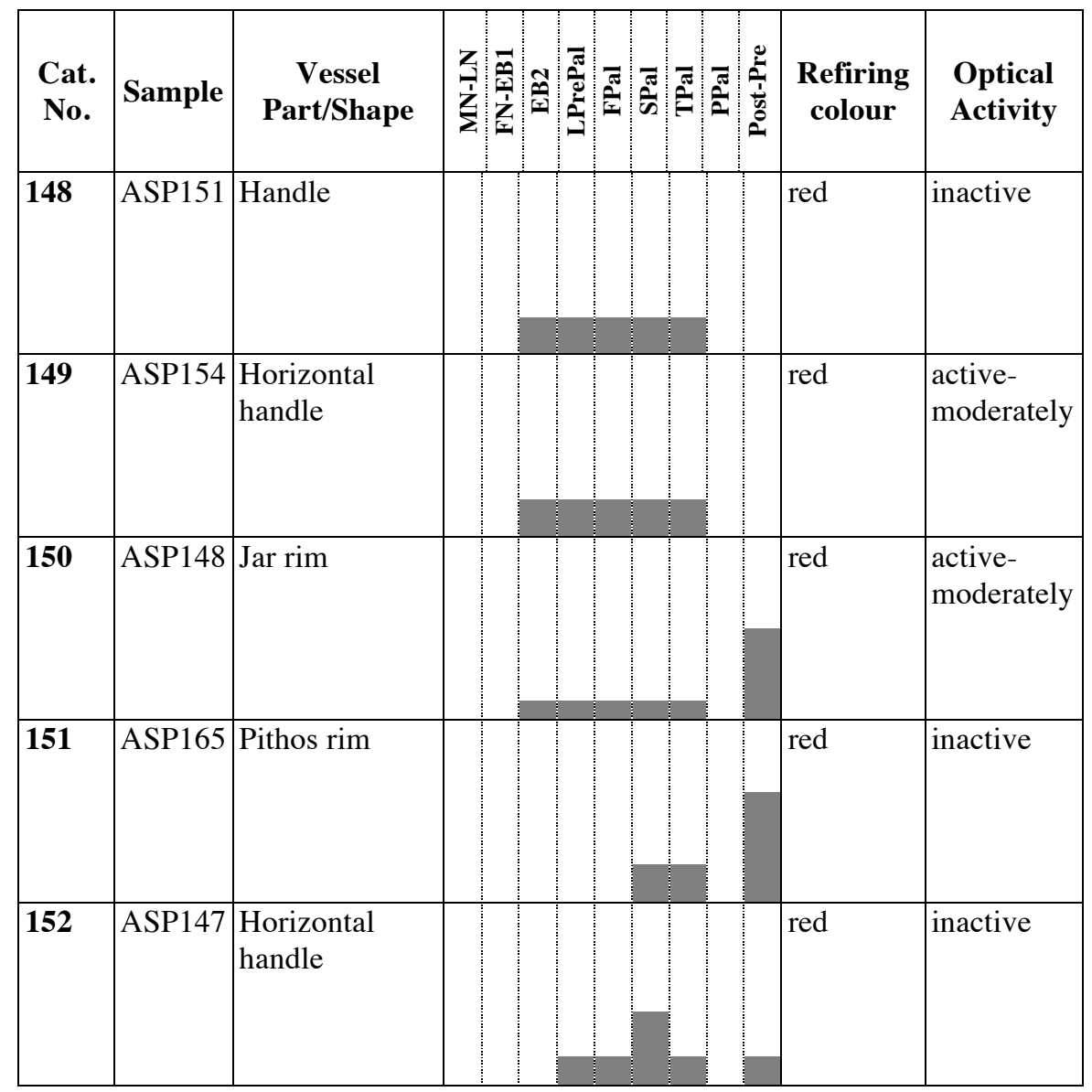

\subsection{MICACEOUS FABRICS (MIC)}

Coarse micaceous fabrics comprise $8-9 \%$ of the overall prehistoric assemblage from Antikythera and include both vessels of earlier (EB2) and later manufacture (SPalTPal).

Macroscopic examination and petrographic analysis coincided in the identification of this fabric, which is incompatible with local geology and matches the Kytheran micaceous fabrics associated with sources in the northern part of that island (Kiriatzi 2003, Broodbank and Kiriatzi 2007, Broodbank et al 2005). Micaceous fabric can be considered imported to Antikythera, while the pottery in this fabric concerns mostly large to medium size storage vessels and medium size cooking pots.

\subsubsection{MIC (FIG.21)}

This is a coarse fabric, consisting of poorly sorted sub-angular to sub-rounded inclusions, unimodal grain size distribution, with maximum grain size $5.2 \mathrm{~mm}$ (mode size $0.5-1 \mathrm{~mm}$ ) and $3-10 \%$ voids. The fabric contains frequent (white) mica schist (commonly bearing sphene and rarely chloritoid) and quartzite fragments, common to 
dominant (white) mica laths, few to common phyllite fragments, quartz and alkali feldspars, few plagioclase, few to very rare sphene and black opaques and very few to rare colourless/pale green/pale blue amphibole. This composition is incompatible with local geology, but resembles very strongly the Kytheran Micaceous fabric (with sphene and amphibole also present), and should therefore be considered an import from Kythera (Kiriatzi 2003, 125; Broodbank and Kiriatzi 2007, 250-1, fig.3c). The fabric is homogeneous in terms of composition, grain size distribution, and refiring colour (red tones). The optical activity of the groundmass varies from active to inactive, suggesting variable original firing temperatures below/around $800^{\circ} \mathrm{C}$, while the firing atmosphere was oxidising.






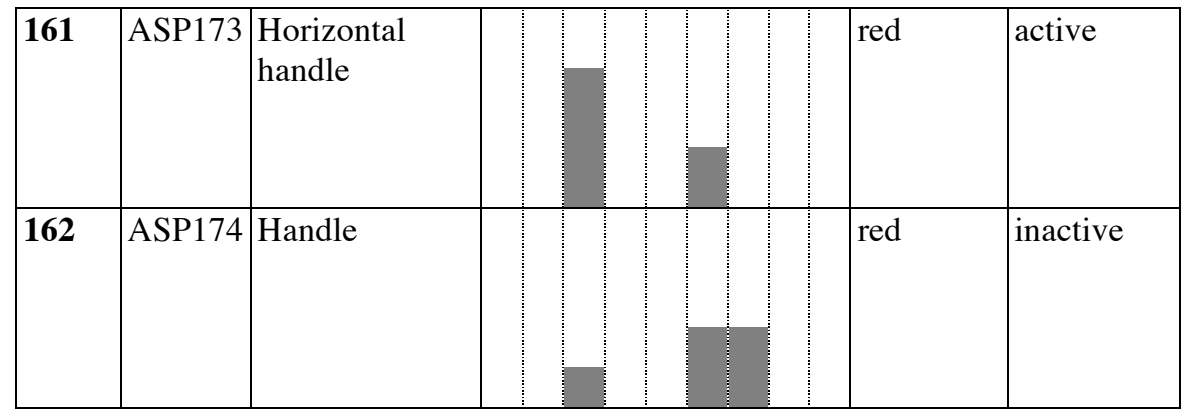

\subsubsection{MIC loner (FIG.21)}

163 possibly contains andalusite as a rock fragments constituent (medium to high relief, colourless in PPL but with fine internal fracture which gives a "dirty" appearance, first order birefringence colours), should be considered as a definite import to Antikythera, but probably has an origin other than Kythera, due to geological incompatibility.

\begin{tabular}{|c|c|c|c|c|c|c|c|c|}
\hline $\begin{array}{l}\text { Cat. } \\
\text { No. }\end{array}$ & Sample & $\begin{array}{c}\text { Vessel } \\
\text { Part/Shape }\end{array}$ & 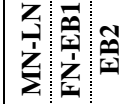 & & क & 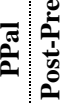 & $\begin{array}{c}\text { Refiring } \\
\text { colour }\end{array}$ & $\begin{array}{l}\text { Optical } \\
\text { Activity }\end{array}$ \\
\hline 163 & ASP171 & $\begin{array}{l}\text { Horizontal } \\
\text { handle }\end{array}$ & & & & & red & active \\
\hline
\end{tabular}

\subsection{CALCAREOUS AND SEDIMENTARY FABRICS (CAS, FIG.22)}

This fabric was identified only microscopically. It is a medium to coarse fabric, consisting of moderately well sorted rounded to sub-angular inclusions, unimodal grain size distribution, with maximum grain size $2 \mathrm{~mm}$ (mode size $0.2-0.5 \mathrm{~mm}$ ) and 2$5 \%$ voids. The fabric contains frequent chert (deriving from primary formations) and micritic limestone fragments, common mudstone / siltstone fragments and Tcfs (clay pellets), few to common quartz and rare schist rock fragments. With present data, local provenance cannot be ruled out for CAS fabric, since this composition is not entirely incompatible to local geology, but neither is it diagnostic. The fabric is homogeneous in terms of composition, grain size distribution, and refiring colour (reddish yellow). The optical activity of the groundmass (inactive) suggests variable original firing temperatures around / exceeding $800^{\circ} \mathrm{C}$, while the firing atmosphere was oxidising to oxidising/reducing.

\begin{tabular}{|c|c|c|c|c|c|c|c|c|c|}
\hline $\begin{array}{l}\text { Cat. } \\
\text { No. }\end{array}$ & Sample & Vessel Part/Shape & 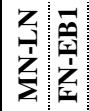 & $\stackrel{\infty}{\alpha}$ & 혼 & के & 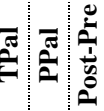 & $\begin{array}{c}\text { Refiring } \\
\text { colour }\end{array}$ & $\begin{array}{l}\text { Optical } \\
\text { Activity }\end{array}$ \\
\hline 164 & ASP83 & Rim of a bowl & & & & & & reddish yellow & inactive \\
\hline
\end{tabular}




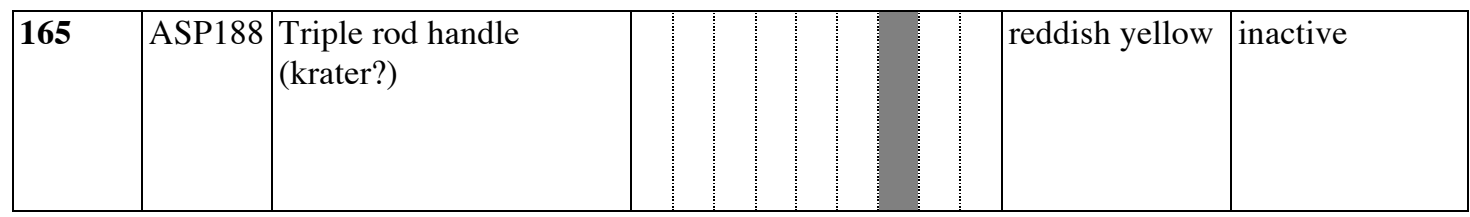

\subsection{LONER FABRICS (OUT, FIG.22)}

166 constitutes a medium fabric of not particularly diagnostic composition regarding provenance, containing quartz, crystalline limestone and chert fragments, along with Tcfs (clay pellets). 167 is medium-coarse, with carbonate inclusions (limestone fragments, rare dolomite crystals and microfossils of the foraminifera and sponge spicules species), presenting some resemblance to Chandler's fabric B1 (Chandler 2001, sample GSE15). 168, 169 and $\mathbf{1 7 2}$ are medium and calcareous fabrics, containing mainly carbonate inclusions (168: limestone fragments and microfossils of the foraminifera species; 169: micritic calcite pellets and lumps and Tcfs (clay pellets), along with few foraminifera, quartz and metamorphic rock fragments; 172: microfossils of the foraminifera species, micritic calcite pellets and few Tcfs / clay pellets), bearing some compositional resemblance to Chandler's fabric B1 (Chandler 2001, samples GSE9, GSE15) but differing in granulometry. $\mathbf{1 7 0}$ is a medium-coarse fabric, containing metamorphic rock fragments (quartzite, mica- and chlorite-bearing schist, oxidised phyllite fragments) and carbonate marls, bearing weak resemblance to Chandler's fabric I (Chandler 2001, sample NR3), which also contains sponge spicules. 171 is a coarse fabric with silicate inclusions (quartz, quartzite fragments, chert, alkali feldspars and plagioclase). All these sherds could either be local products or imports from western Crete, whereas $\mathbf{1 7 3}$ and $\mathbf{1 7 4}$ should be considered definite imports. $\mathbf{1 7 3}$ is coarse, containing quartz, metamorphic rock fragments (quartzite, shale/phyllite, mica schist) and limestone fragments and few clay pellets; this composition is not compatible to the Antikythera geology, and might be linked to that of western Crete. 174 is medium-coarse, containing plagioclase, shale/phyllite fragments, tuffs / igneous rock fragments and carbonate marls; this composition is incompatible with local geology, therefore this sample should be an import to Antikythera.

\begin{tabular}{|c|c|c|c|c|c|c|c|c|}
\hline $\begin{array}{l}\text { Cat. } \\
\text { No. }\end{array}$ & Sample & $\begin{array}{c}\text { Vessel } \\
\text { Part/Shape }\end{array}$ & 学案 & 웡 & & สิ & $\begin{array}{c}\text { Refiring } \\
\text { colour }\end{array}$ & $\begin{array}{l}\text { Optical } \\
\text { Activity }\end{array}$ \\
\hline 166 & ASP79 & $\begin{array}{l}\text { Cooking pot } \\
\text { leg }\end{array}$ & & & & & red & $\begin{array}{l}\text { active- } \\
\text { moderately }\end{array}$ \\
\hline 167 & ASP109 & $\begin{array}{l}\text { Rim/handle } \\
\text { (ladle) }\end{array}$ & & & & & red & $\begin{array}{l}\text { moderately } \\
\text {-slightly }\end{array}$ \\
\hline 168 & ASP190 & $\begin{array}{l}\text { Kylix base and } \\
\text { stem }\end{array}$ & & & & & $\begin{array}{l}\text { reddish } \\
\text { yellow }\end{array}$ & moderately \\
\hline
\end{tabular}




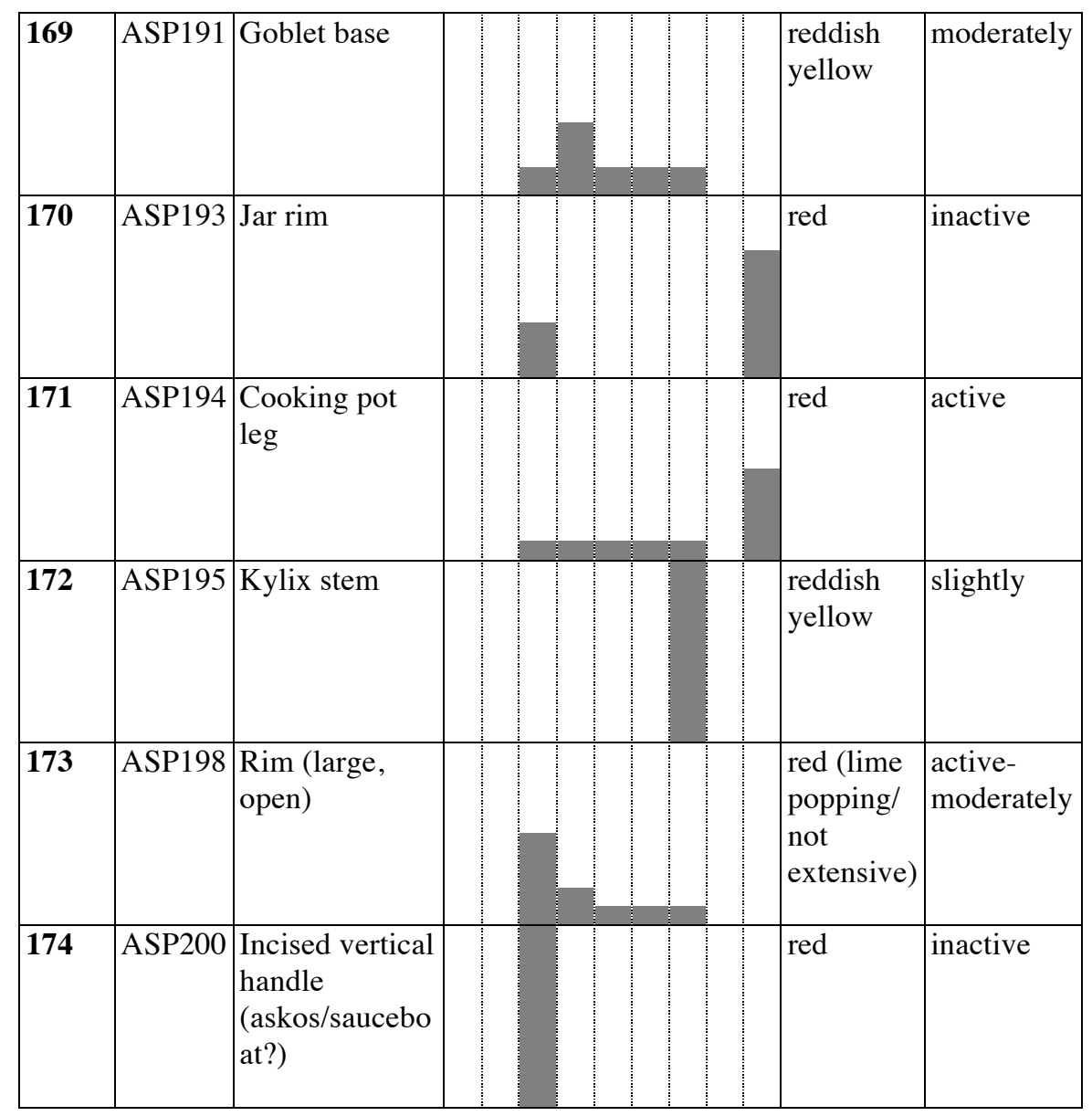




\section{CHRONOLOGICAL COMMENTS}

\subsection{LATE NEOLITHIC TO EARLY BRONZE I}

With the possible exception of one bowl fragment (131), our earliest pottery evidence is from the FN-EB1 phase, including a cheesepot (175), a ladle (167), various jars with thick flat bases (172) and/or sharply raised ledges/bands (177), as well as many small, highly-battered body sherds in GROb or similar fabrics (such fabrics usually accounting for more than $80 \%$ of the pottery observed on an FN-EB1 surface scatter). The Grog tempered fabrics have more parallels with the analogous fabrics identified on Kythera rather than those in western Crete (Nodarou 2003 and pers.comm.). This could be considered as an indication that the Antikytheran ones were either imported from Kythera or made locally, although more comparanda are needed from Crete to clarify the picture. Overall associations with Kythera (Broodbank and Kiriatzi 2007) as well as with Crete (Nerokourou: Vagnetti et al 1989) are implied not only by common technological practices (grog-tempering), but by overall vessel typology.

\subsection{EARLY BRONZE 2}

There is a wide range of pottery that can convincingly be dated to the EB2 period, the best comparanda for which come from deposits $\alpha-\beta$ at Kastri on Kythera (Coldstream and Huxley 1972, 77-91, figs.35-6, pls.16-20; Broodbank and Kiriatzi 2007, 248-55, figs.3-5) and from EMIIA Nopigeia in western Crete (Karantzali 1996, 89-91, figs.95101; Nodarou 2003). With the latter assemblages in mind, there are good examples from Antikythera of sauceboats (e.g. 77, 178), handles from various pouring shapes with either deep incisions down the middle or diagonal slashes (e.g. 80, 135, 163, 174), saucers (e.g. 173), t-rim bowls (e.g. 164), impressed herringbone decoration (e.g. 177, 179) and medium-sized jars or pithoi with flaring rims and impressed bands (e.g. 64). Some of the EB2 pottery is certainly imported from Kythera (e.g. 177-8) and probably also from western Crete, while other examples may have been locally made on the island (e.g. the EB2 grog-tempered fabrics, see below). Sauceboat $\mathbf{7 7}$ is particularly interesting as the shape is similar to west Cretan examples, with a globular body and low foot, but the surface treatment, incised nicks, and fabric is not typically west Cretan (where black-slipped but otherwise undecorated examples in a very fine calcareous clay with small quartz fragments are typical: E. Nodarou pers.comm.). There are also shapes that we find on Kythera or in western Crete that are so far absent or extremely rare in the Antikythera material. Some shapes such as pyxides, we might not expect to find in surface material, but it is interesting that, with the unlikely exception of an EB2 sherd with deep herringbone-impressed decoration and very little curvature (179), there are no identified examples of portable hearths (such as those found elsewhere with kerbschnitt decoration: e.g. Karantzali 1996, fig.98, 101; Coldstream and Huxley 1972, 83, $\alpha 98$, pl.17.98; Broodbank 1999, fig.12 bottom row, second from right) or externally-scored ware (e.g. Karantzali 1996, fig.95; Coldstream and Huxley 1972, 80, $\alpha 46$, pl. 16.46). The full suite of fabrics used in EB2 is hard to discern entirely, but it is interesting that diagnostic EB2 shapes such as $\mathbf{7 7}$ are found in the GROb fabric which extends the chronological range of this fabric beyond that documented on Kythera (where it appears to be distinctive of FNEB1 only). More generally various grog-tempered fabrics with many TCFs seem to be 
a feature of EB2 Antikythera and may well be locally made, given that they are compatible with local geology and without contemporary petrographic matches on either EB2 Kythera or western Crete. Alongside these grog tempered fabrics, sand and calcite tempering is clearly present, with a few possible but uncertain examples of mudstone tempered and phyllite tempered sherds at this stage as well. A suite of related fabrics with a very clean clay matrix (e.g. SATc, CALb) may also begin to appear in this phase though this remains difficult to verify conclusively.

\subsection{LATE PREPALATIAL TO FIRST PALACE PERIOD}

While these two phases have been separately defined as part of our working chronological scheme, in practice, they remain problematic to distinguish consistently on Antikythera. It is possible that the island was not always settled over this time period, but the more fundamental issue is a lack of sufficient diagnostic indicators amongst the recovered pottery. Our best comparanda are those from deposit $\gamma-\delta$ at Kastri on Kythera (Coldstream and Huxley 1972, 91-8, figs.37-8; pls.21-2; Broodbank and Kiriatzi 2007, 251-55, figs.5-6) and brief publication of the finds from Psathi Kydonias (Mitilinaiou 1997-8) and Khamalevri (Andreadaki-Vlasaki 1997). In some ways, the late Prepalatial should be easier to recognise than the First Palace period, with the latter hardly known at all in western Crete and with very few, clear coarseware diagnostics from Kythera. For the Late Prepalatial, some of the finds from Antikythera, such as a few crested and pushed through handles (e.g. 138, 180) and a fineware base from a possible goblet (e.g. 169) are potentially comparable to finds of this date from neighbouring areas, but other classes of pottery that we might expect, such as punctured or barbotine decoration or bowls with incurved rims, are missing or far less obvious. For the First Palace period, there are several possible carinated cup fragments from the Antikytheran scatters (e.g. 181) and a range of circumstantial evidence of widespread activity, but the lack of excavated MM assemblages from western Crete and the small size of the relevant deposit from Kastri on Kythera means that we currently have very few clearly diagnostic First Palace indicators. More broadly, certain large tripod cooking pot legs in calcite tempered fabrics or flaring bowls in calcite or grog-tempered fabrics are also probably of Late Prepalatial to First Palace date, as are a small number of Kytheran sand tempered sherds (e.g. SATd), but few if any are entirely diagnostic one of these periods in particular. Finally, as above, we suspect that the group of SATc/CALb fabrics with a distinctive finer groundmass is also likely to be of EB2, late Prepalatial and/or First Palace, but at present cannot be more specific.

\subsection{SECOND PALACE PERIOD}

By contrast to the late Prepalatial and First Palace periods, the Second Palace period can be identified via a fairly wide range of diagnostic sherds and is a dominant or major component at many different scatters across the island. The most useful regional comparanda for this period come from the well published sites of Kastri on Kythera (Coldstream and Huxley 1972, deposits $\varepsilon, \zeta, \eta, \mu, v, \xi$ and partly $\omega$ ) and Nerokourou in central-western Crete (Kanta and Rocchetti 1989), and Vrises (Efstratiou 1974) and observations made by Kytheran and western Cretan surface surveys (Broodbank 1999; Moody 1985, 1987; Moody et al 2003). Although we have not catalogued the limited number of Second Palace finewares here, it is worth noting the presence of some definite Vapheio cups, hemispherical or stemmed cups, tumblers 
and typical Second Palace painted decorations amongst the collected material. In addition, various imported Kytheran coarsewares in micaceous fabric are good indicators of this period, including tripod cooking pots (e.g. 156-158, though these continue into the Third Palace period as well), pithoi (e.g. 155) and medium to largesize vessels with ribbed decoration (e.g. 159). More broadly, medium to large sized jars in a variety of fabrics can be clearly dated to the Second Palace period based on rim forms that are flat-topped and squared off (e.g. 103) and/or inset for a lid (e.g. 11, 39, 155). In addition, there are many instances of medium-sized jars and pithoi decorated with plastic bands of overlapping discs produced by pinching the plastic band vertically and rolling to the right or left (e.g. 182-3). On Crete, these are found on MMIII-LMI jars and ovoid pithoi from Crete (Christakis 2005, 26, rope group V and forms 4 and 6, fig.2) and on Antikythera, this decorative scheme is also found immediately below the plain rims of large open bowls in grog-tempered fabrics (e.g. 184, though in other examples the bands are not articulated as clear overlapping discs). There are also other medium-sized storage jars and pithoi that have one or more plastic bands decorated with a series of parallel curved lines, probably impressed with a sharp-edged tool (e.g. 101, 105). On Crete, these are common decorative schemes on western Cretan ovoid pithoi, especially during MMIII-LMI, but continuing as late as LMIII (Christakis 2005, bands 71-4). There are over a dozen fragments with this decoration from Antikythera (from at least half a dozen different pithoi and medium sized jars), all from scatters of likely Second Palace date and all in mudstone-tempered fabrics that appear to be imports from western Crete or local products made according to western Cretan traditions rather than imports from Kythera.

\subsection{THIRD PALACE PERIOD}

Kylix stems are one of the traditional ways to identify this period in surface material, not least because they are so physically robust as surface artefacts, but only nine examples have been found by the survey (e.g. 168, 172, 184-5). Many of these are hollow in section and hence showing greater affinity with Cretan foot-forming methods than contemporary mainland traditions (e.g. Hallager 2003, 212). However, at least five also exhibit a horizontal pre-firing hole through the top of the stem near the bowl (e.g. 184-5), which may have been meant to expedite the drying out of this thick mass of wet clay during manufacture. In any case, this feature is exceptionally rare among Cretan kylikes, but has been noted amongst the larger examples from Pylos (Blegen and Rawson 1966, 370-1, pls.361.356, 363.337-8, 629 364.338,367; J. Hruby pers.comm; although the Pylian stems are not hollow). Another interesting fineware sherd from a Third Palace period scatter is a triple rod handle that is probably from an amphoroid krater (165) of a type known from LMIIIA2-B contexts in western Crete (Andreadaki-Vlasaki 1994-6, pl.2b bottom left; Hallager 2000, pl.76d, 79b.4; Hallager 2003, 220, pls.95d.1, 102d.8, 110f.10, 124c.2; or less likely a leg such as Blegen and Rawson 1966, pl.395-6.457). This and the long stem of at least one of the kylikes (185) are also indications that prehistoric occupation on Antikythera continued at least as late as LMIIIA2-B. In addition to these rare finewares, sustained study of the coarsewares also suggests that certain trianguloid jar rims (e.g. 87), rounded section tripod cooking pot legs and certain combinations of fabrics (e.g. assemblages with many outlying sand-tempered and mudstone fabrics, Kytheran micaceous sherds and calcareous and sedimentary fabric) can also be used to distinguish this period from earlier palatial assemblages. 


\section{DISCUSSION}

The prehistoric pottery from intensive survey on Antikythera is part of a wider set of evidence that allows us to suggest changing patterns of human activity on the island over some 7000 years. Knapped stone evidence points reasonably firmly to activity on the island from at least the $5^{\text {th }}$ millennium BC (e.g. Bevan et al 2008, 33-4, fig.4) and the island seems to have seen seasonal and/or semi-permanent exploitation during some parts of the LN and FN-EB1 periods. In particular, it is the FN-EB1 surface scatters that are comparatively easy to identify on the island, because the ceramics, though few in number and poorly preserved in any given location, are often closely associated with worked obsidian and white chert artefacts (and the latter are only found rarely with later prehistoric material). Thereafter, the survey results suggest that that there is a shift in the nature and location of human activity on the island sometime during the EB2 phase. At present, it remains difficult to determine whether, immediately prior to this, there was a gap in the regular exploitation of the island, but in any event, we can associate clearly diagnostic EB2 sherds with different, more agriculturally favourable locations in the landscape and often with areas that show continuity or repeated use into the $2^{\text {nd }}$ millennium BC. While the Late Prepalatial and First Palace periods remain difficult to isolate as clearly as we might wish (see above), the impression is that similar kinds of settlement patterns exist for at least some of this time. By the Second Palace period, over twenty surface scatters suggest loose groups of dispersed farmsteads across the island, some of which clearly began in the preceding Late Prepalatial or First Palace periods, but others of which were newly inhabited. In the Third Palace period, a similar general settlement pattern continues, even if sometimes in new locations, while the lack of any clearly diagnostic Postpalatial finds (e.g. none obviously similar to those found on Cretan sites; Nowicki 2000, 214-22, 267-9, pls.xxii-liv) suggests that the island was thereafter abandoned and did not see substantial habitation again until the late $4^{\text {th }}$ century BC.

For at least the SPal and TPal periods, if not before, the pottery includes a wide range of pithoi, medium-sized jars, open bowls, cooking pots and finewares, etc with most of these present on any given surface scatter. Such vessels fall into three major functional categories, for: (i) serving and consumption, (ii) food processing and heating and (iii) medium- or large-scale storage and, although final spatial analysis of the distribution of these finds is still ongoing, our strong impression is that most EB2TPal scatters represent permanent farmsteads, with any slight differences in the recovered proportions of these categories largely due to post-depositional taphonomy rather than functional differentiation. However, it is also worth mentioning two further activities that seem to have been present on prehistoric Antikythera: textile manufacture and apiculture. The former is documented by at least two surviving fragments from discoid loomweights of typical Cretan size and type (45, also 80252195-S-1). These are made in sand-tempered fabrics and could either have been made locally or imported from western Crete, but they certainly attest to the importance of Cretan-style weaving practices on the island during the Bronze Age. ${ }^{7}$

${ }^{7}$ It is also worth noting in passing that no examples have been found during the Antikythera survey of any of the horizontal perforated bar weights that are relatively common on Kythera and are assumed to be associated with some kind of specialised 
Possible evidence for semi-specialised apiculture on Antikythera is provided by more than 80 sherds with incised lines and/or finger impressions on their interiors (e.g. 3, $12,14,17,19,28,65)$. There are only a few published parallels for these finds on Crete (e.g. Hallager 2000, 161, pl.57 f.16; Hallager 2003, 242-3, pls.76, 101b; Kanta and Rocchetti 1989, 312, figs. 64.482, 71.537, 71.539 and 76.572; Watrous 1992, fig.22.439; Melas 1999, pls.cviiia), though others are present in unpublished coarseware assemblages (C. Knappett pers. comm.). The fragments all seem to come from wide-mouthed, medium-sized conical jars that, on their exteriors, often have both horizontal handles and a finger-impressed band placed below the rim. In addition, parallel and/or cross-hatched incised lines are typically found on the bottom third of the vessel interior and a dense group of finger impressions is sometimes applied to the interior base. This interior treatment may conceivably have been meant to create a grinding surface for the jar's contents, but Manolis Melas' suggestion (1999) is arguably more plausible: that these vessels were beehives, for which the interior scoring and impressions provided purchase for the honeycomb (hence similar in concept to later Classical to Roman pottery beehives). Such vessels are found with almost every First to Second Palace period scatter on Antikythera (with possible but more equivocal evidence for the EB2, late Prepalatial and/or Third Palace periods). The absence of any examples in demonstrably non-local fabrics (e.g. phyllite/shale, micaceous or outlier fabrics) suggests that they may have been locally made, while the sheer number found at different sites on Antikythera contrasts with Kythera (where no examples are known at present) and Crete (where they are fairly rare, if currently underestimated). Interestingly, the only places where similarly abundant examples have been noted are the scattered rural farmsteads on Kasos and Karpathos (Melas 1999), perhaps suggesting that apiculture was particularly important for the communities living on the otherwise fairly marginal islands to either end of Crete (excluding Kythera).

The wider social and economic context for Bronze Age settlement and material culture on Antikythera will be discussed at greater length elsewhere (Bevan and Conolly in prep), but it is worth making some final comments here about how the Antikytheran fabric analysis informs our wider understanding of prehistoric potting traditions in the south-west Aegean. This analysis demonstrates a regional pattern of trade in coarsewares (both open shapes and containers) in which there are definite pottery imports from Kythera (namely the MIC, SATd and MUTc fabric sub-groups which macroscopic assessment suggests comprise ca.10\% of the overall assemblage) and western Crete (at the very least $3 \%$ of the overall assemblage represented by the SHAa-e sub-groups, with parallels in the Chania area, and probably much more, especially of the CAL, MUT and SAT fabric groups), but none clearly from central or eastern Crete and few as yet no clearly identifiable examples from more distant places.

It is the balance between local and west Cretan production that remains hardest to define however, with a large number of fragments belonging to sand, grog, mudstone and calcite tempered fabrics that, in the present state of our knowledge, could equally

textile manufacture (e.g. Broodbank 1999: fig.9 bottom; Coldstream and Huxley 1972: $\pi 43,167-9, \zeta 158, \varepsilon 110, \omega 374 \mathrm{~A}-\mathrm{C}$, figs.59-61, pls.60-1). 
come from Antikythera or western Crete (a group with floating provenance that makes up over $80 \%$ of the overall assemblage). Several of the main fabric subgroups have properties that cross-cut different tempering strategies and may suggest the same provenance. For example, on one hand, the same clay base seems to have been used for SATa, GROa and MUTa (possibly also CALa), namely a silty groundmass, while, on the other hand, a fine and strongly birefrigent groundmass is typical for SATc and CALb. For the latter, no hypothesis can be made about provenance on present evidence, but the silty groundmass of the larger first group has parallels in western Crete and particularly the Kissamos area (Chandler 2001, 388-389; P. Day, J. Moody and E. Nodarou pers.comm.), from where all or part of this pottery may have been imported. Ultimately, we suspect that some degree of on-island manufacture is likely, whether by permanently resident or visiting potters, but whatever the case, the Antikytheran material clearly indicates a potting tradition that was heavily dependant on the nearest neighbouring regions, and in particular on western Crete. It is also probable that both the potentially local pottery as well as the imported material covered a wide range of shapes and functions. The definite imports include pithoi, cooking pots as well as small to medium size pots for storage, transport and consumption, while the potentially local pottery comprises cooking pots, medium to small size vessels for storage, transport and consumption, but perhaps fewer pithoi.

In terms of clay paste preparation, tempering seems to have been the prevailing practice during prehistory on Antikythera and the four major fabric groups are evidently tempered with a variety of materials such as sand, grog, crushed limestone or mudstone. These fabrics may well have the same provenance, but they present technological diversification in terms of clay paste preparation and signify the specialised use of certain pastes (e.g. CAL for cooking-pots) as well as the coexistence of multiple potting traditions. Sand and mudstone tempering is a common practice in Crete throughout the Bronze Age as it also seems to be on Antikythera, while calcite tempering probably begins fairly early on Antikythera but is most related to EB2 to First Palace period production as appears to be the case in western Crete (while it is far less common on Kythera, associated with cooking-pots). The widespread practice of grog-tempering on Antikythera is particularly interesting, primarily because such grog tempered fabric recipes were very uncommon in neighbouring areas after FN-EB1. The persistence of this practice on Antikythera may well be a response either to limited local availability of raw materials (e.g. beach sand, calcite or mudstone) and/or a surviving feature of a local tradition that was reproduced, incorporating new fashions concerning the appearance of the vessels (shape, decoration) while at the same time maintaining much older fabric recipes.

Comparisons to neighbouring Kythera shed light on the different role of the two islands in regional networks since much higher variation in fabric, as well as higher frequency of imports characterise the Antikythera prehistoric pottery. In any case, the above discussion, we hope, emphasises the great potential offered by combined macroscopic, microscopic and comparative approaches to coarseware pottery in the Mediterranean, and underlining the diversity of production, exchange and consumption patterns that supported Antikythera's human communities during prehistory.

\section{CATAlOgUE}


1 (petrography sample ASP1; 2021-8-1-95-1) 707269E, 3972816N

General: Thin, everted rim from a large diameter, flaring bowl. Possible slip and burnish on exterior?

Fabric: SATa Date: 20\% EB2, 40\% LPrePal 30\% FPal, 10\% SPal

2 (petrography sample ASP2; 5028-2-1-15-1) 707931E, 3973433N

General: Body fragment with the beginning of a plastic impressed band, or possibly a lug. Medium to large-sized vessel.

Fabric: SATa Date: 50\% FN-EB1, 20\% EB2, 10\% LPrePal, 10\% FPal, 10\% SPal

3 (petrography sample ASP3; 10028-42-1-45-2) 706674E, 3971464N

General: Base fragment (diameter $25 \mathrm{~cm}$ ) with finger impressions on the interior.

Fabric: SATa Date: 10\% EB2, 10\% LPrePal, 30\% FPal, 30\% SPal, 20\% TPal

4 (petrography sample ASP6; 9034-29-1-75-1) 709014E, 3969597N

General: Rim sherd with plastic decoration on shoulder.

Fabric: SATa Date: 10\% LPrePal, 20\% FPal, 30\% SPal, 40\% TPal

5 (petrography sample ASP7; 3449-12-1-25-1) 707872E, 3971584N

General: Rim sherd (diameter ca. $15 \mathrm{~cm}$ ) and vertical ovoid handle scar, probably from an oval-mouthed amphora.

Fabric: SATa Date: $45 \%$ FPal, 45\% SPal, $10 \%$ TPal (scatter is [FPal-]Spal)

6 (petrography sample ASP9; 8425-2105-S-4) 708430E, 3972106N

General: Horizontal handle, rounded ovoid in section but rapidly tapering; attachment of conical wedge of clay visible against body.

Fabric: SATa Date: 10\% EB2, 25\% LPrePal, 25\% FPal, 25\% SPal, 15\% TPal (scatter is [FPal]-Spal)

7 (petrography sample ASP11; 6795-2245-S-2) 706798E, 3972248N

General: Rim sherd of a large open vessel with a plastic impressed band directly below a plain rim.

Fabric: SATa Date: 20\% EB2, 20\% LPrePal, 20\% FPal, 30\% SPal, 10\% TPal

8 (petrography sample ASP15; 8405-2095-S-3) 708407E, 3972095N

General: Rim sherd with plastic impressed band under rim.

Fabric: SATa Date: 30\% EB2, 30\% FPal, 40\% SPal (scatter is [FPal-]SPal)

9 (petrography sample ASP23; 8795-9925-S-1) 708791E, 3969650N

General: Two plastic impressed bands on the exterior of a large vessel, probably a pithos.

Fabric: SATa Date: $40 \%$ FPal, $60 \%$ SPal

10 (petrography sample ASP24; 7955-2155-V-3) 707953E, 3972151N

General: Body sherd, differentially tempered on the exterior.

Fabric: SATa Date: 20\% EB2, 20\% LPrePal, 20\% FPal, 20\% SPal, 20\% TPal

11 (petrography sample ASP26; 7795-1875-S-2) 707791E, 3971873N

General: Incurved rim with ledge from a pithos.

Fabric: SATa Date: 20\% FPal, 60\% SPal, 20\% TPal 
12 (petrography sample ASP27; 8146-29-1-65-1) 706547E, 3973264N

General: Body fragment of a medium to large vessel with incised lines on interior. Fabric: SATa Date: $20 \%$ EB2, 20\% LPrePal, 20\% FPal, 20\% SPal, 20\% TPal

Parallels: Melas 1999

13 (petrography sample ASP32; 9645-0345-S-1) 709641E, 3970341N

General: Sub-rectangular sectioned handle (probably horizontal); crackly surface.

Fabric: SATa Date: 20\% EB2, 20\% LPrePal, 20\% FPal, 20\% SPal, 20\% TPal (scatter is FPal-SPal)

14 (petrography sample ASP34; 4020-17-1-5-2) 706682E, 3972757N

General: Body sherd from a medium to large vessel, with incised lines on interior.

Fabric: SATa Date: 20\% EB2, 20\% LPrePal, 20\% FPal, 20\% SPal, 20\% TPal

15 (petrography sample ASP36; 8805-9925-S-5) 708805E, 3969645N

General: Medium thin, ovoid leg from a tripod cooking pot.

Fabric: SATa Date: 20\% EB2, 20\% LPrePal, 20\% FPal, 20\% SPal, 20\% TPal

16 (petrography sample ASP37; 8795-9935-V-2) 708796N, 3969655E

General: Medium thin, ovoid leg from a tripod cooking pot.

Fabric: SATa Date: 20\% EB2, 20\% LPrePal, 20\% FPal, 20\% SPal, 20\% TPal

17 (petrography sample ASP180; 8785-9915-S-2) 708787E, 3969673N

General: Flaring base fragment with incised lines on the interior. Overfired.

Fabric: SATa Date: 20\% EB2, 20\% LPrePal, 20\% FPal, 20\% SPal, 20\% TPal

18 (petrography sample ASP182; 8115-2375-S-1) 708115E, 3972376N

General: Rim fragment of a jar with a plastic impressed band beneath the rim.

Fabric: SATa Date: 20\% EB2, 10\% LPrePal, 10\% FPal, 50\% SPal, 10\% TPal

19 (petrography sample ASP197; 6705-1485-S-4) 706703E, 3971486N

General: Body fragment with incised lines on the interior.

Fabric: SATa Date: 20\% EB2, 20\% LPrePal, 20\% FPal, 20\% SPal, 20\% TPal

20 (petrography sample ASP40; 12393-17-1-15-5) 710074E, 3967117N

General: Fragment of a jug or narrow-necked jar, with an ovoid to sub-rectangular handle coming off the rim.

Fabric: SATa Date: 10\% EB2, 15\% LPrePal, 20\% FPal, 20\% SPal , 15\% TPal, 20\%

Post-prehistoric

21 (petrography sample ASP8; 8805-9935-S-1) 708805E, 3969643N

General: Rim fragment of a pithos.

Fabric: SATa Date: $40 \%$ FPal, $40 \%$ SPal, $20 \%$ TPal

22 (petrography sample ASP92; 8005-2155-S-2) 708010E, 3972159N

General: Rim fragment of a pihtos, with a plastic impressed band under the rim.

Fabric: SATa Date: 30\% EB2, 30\% FPal, 40\% SPal

23 (petrography sample ASP122; 4210-39-1-65-1) 706734E, 3972296N 
General: Small ovoid leg of a tripod cooking pot.

Fabric: SATa Date: 20\% EB2, 20\% LPrePal, 20\% FPal, 20\% SPal, 20\% TPal (scatter is FPal-SPal)

24 (petrography sample ASP127; 6385-3455-S-1) 706383E, 3973455N

General: Small ovoid leg of a tripod cooking pot.

Fabric: SATa Date: 20\% EB2, 20\% LPrePal, 20\% FPal, 20\% SPal, 20\% TPal

25 (petrography sample ASP136; 6325-3425-S-1) 706323E, 3973427N

General: Small leg from a tripod cooking pot; ovoid near the top and more cylindrical towards the bottom

Fabric: SATa Date: 20\% EB2, 20\% LPrePal, 20\% FPal, 20\% SPal, 20\% TPal

26 (petrography sample ASP4; 3482-19-1-5-1) 708011E, 3972244N

General: Body fragment with an abraded plastic impressed wavy band on exterior of medium sized vessel.

Fabric: SATb Date: 20\% EB2, 20\% LPrePal, 20\% FPal, 20\% SPal, 20\% TPal

27 (petrography sample ASP10; 6735-1945-S-1) 706730E, 3971942N

General: Rim fragment of a large open bowl or pan. Orange exterior and grey core.

Fabric: SATb Date: $40 \%$ EB2, 15\% LPrePal, 15\% FPal, 15\% SPal, 15\% TPal

28 (petrography sample ASP12; 9635-0365-S-5) 709637E, 3970365N

General: Base fragment from a pithos. Two rows of finger impressed decoration on base.

Fabric: SATb Date: 10\% EB2, 10\% LPrePal, 30\% FPal, 30\% SPal, 20\% TPal (scatter is FPal-SPal)

29 (petrography sample ASP14; 9375-0865-V-5) 709371E, 3970864N

General: Rim fragment of a bowl (diameter ca.20cm) with diagonal incisions on the interior.

Fabric: SATb Date: 20\% EB2, 20\% LPrePal, 20\% FPal, 20\% SPal, 20\% TPal

30 (petrography sample ASP18; 7855-1575-S-1) 707857E, 3971578N

General: A large, thin ovoid leg of a tripod cooking pot.

Fabric: SATb Date: 10\% EB2, 10\% LPrePal, 35\% FPal, 35\% SPal, 10\% TPal (scatter is [FPal-]SPal)

31 (petrography sample ASP28; 3456-14-1-95-1) 708035E, 3971786N

General: Rim fragment of pithos.

Fabric: SATb Date: 10\% EB2, 10\% LPrePal, 30\% FPal, 40\% SPal, 10\% TPal

32 (petrography sample ASP29; 8105-1685-S-1) 708103E, 3971687N

General: Rim fragment with scar from the attachment of a vertical handle.

Fabric: SATb Date: 10\% EB2, 10\% LPrePal, 20\% FPal, 30\% SPal, 30\% TPal

33 (petrography sample ASP30; 8795-9935-V-3) 708799E, 3969656N

General: Body sherd of a large vessel with two plastic impressed bands on the exterior.

Fabric: SATb Date: $40 \%$ FPal, $60 \%$ SPal 
34 (petrography sample ASP35; 8805-9925-S-4) 708804E, 3969649N

General: Probably a thin ovoid leg from a tripod cooking pot or possible a handle

Fabric: SATb Date: 10\% EB2, 10\% LPrePal, 35\% FPal, 35\% SPal, 10\% TPal

35 (petrography sample ASP181; 7335-3425-S-2) 707337E, 3973421N

General: Rim fragment with a plastic band below rim, possibly impressed.

Fabric: SATb Date: 25\% EB2, 10\% LPrePal, 20\% FPal, 20\% SPal, 25\% TPal

36 (petrography sample ASP186; 6755-1475-S-14) 706754E, 3971476N

General: Thin ovoid leg from a tripod cooking pot, tapering rapidly to a thin point. Possible reddish brown slip on the exterior.

Fabric: SATb Date: 20\% EB2, 20\% LPrePal, 20\% FPal, 20\% SPal, 20\% TPal

37 (petrography sample ASP199; 6745-1475-V-1) 706748E, 3971471N

General: Rim fragment from a bridge-spouted jar or possibly a medium coarse sauceboat?

Fabric: SATb Date: 30\% EB2, 20\% LPrePal, 30\% FPal, 20\% SPal

38 (petrography sample ASP31; 6795-2225-S-2) 706793E, 3972220N

General: Medium to large-sized thin ovoid leg from a tripod cooking pot. Poorlymixed clay.

Fabric: SATb Date: 20\% EB2, 20\% LPrePal, 20\% FPal, 20\% SPal, 20\% TPal (scatter is FPal-SPal)

39 (petrography sample ASP5; 4219-17-1-5-2) 706810E, 3972028N

General: Pithos rim. Very well fired.

Fabric: SATb Date: $100 \%$ SPal

40 (petrography sample ASP184; 6735-1465-S-1) 706740E, 3971463N

General: Thick, medium-sized ovoid leg from a tripod cooking pot.

Fabric: SATb Date: 20\% EB2, 20\% LPrePal, 20\% FPal, 20\% SPal, 20\% TPal

41 (petrography sample ASP75; 3693-9-1-35-1) 708522E, 3969885N

General: Handle (probably horizontal) of medium sized vessel; three incised notches.

Fabric: SATb Date: 20\% EB2, 20\% LPrePal, 20\% FPal, 20\% SPal, 20\% TPal

42 (petrography sample ASP134; 7325-3415-S-2) 707322E, 3973412N

General: Very large ovoid leg of a tripod cooking pot.

Fabric: SATb Date: 20\% EB2, 20\% LPrePal, 20\% FPal, 20\% SPal, 20\% TPal

43 (petrography sample ASP19; 6725-1945-S-3) 706722E, 3971945N

General: Either a vertical handle from a vessel such as an askos or a very small, thin ovoid leg from a tripod cooking pot. Perhaps a vestigial vertical groove on one side.

Fabric: SATc Date: 40\% EB2, 20\% LPrePal, 20\% FPal, 20\% SPal

44 (petrography sample ASP20; 8005-2205-S-1) 708001E, 3972207N

General: Medium-sized, thin ovoid leg from a tripod cooking pot.

Fabric: SATc Date: 20\% EB2, 20\% LPrePal, 20\% FPal, 20\% SPal, 20\% TPal 
45 (petrography sample ASP21; 7935-2145-S-2) 707933E, 3972145N

General: Fragment of a discoid loomweight (diameter $10 \mathrm{~cm}$ ); abraded on one side.

Fabric: SATc Date: 20\% EB2, 20\% LPrePal, 20\% FPal, 20\% SPal, 20\% TPal

46 (petrography sample ASP41; 11089-4-1-65-4) 707797E, 3971532N

General: Small, ovoid leg from a tripod cooking pot.

Fabric: SATc Date: 20\% EB2, 20\% LPrePal, 20\% FPal, 20\% SPal, 20\% TPal

47 (petrography sample ASP48; 8025-2155-V-1) 708025E, 3972157N

General: Medium-sized, ovoid leg from a tripod cooking pot.

Fabric: SATc Date: 20\% EB2, 20\% LPrePal, 20\% FPal, 20\% SPal, 20\% TPal

48 (petrography sample ASP185; 6745-1465-S-4) 706742E, 3971461N

General: Medium sized, rounded ovoid leg from a tripod cooking pot.

Fabric: SATc Date: 20\% EB2, 20\% LPrePal, 20\% FPal, 20\% SPal, 20\% TPal

49 (petrography sample ASP187; 7755-1975-V-1) 707756E, 3971970N

General: Medium sized leg of a tripod cooking pot with a rounded ovoid section at top tapering to a rounded point at the bottom.

Fabric: SATc Date: 20\% EB2, 20\% LPrePal, 20\% FPal, 20\% SPal, 20\% TPal

50 (petrography sample ASP177; 3621-42-1-25-3) 708365E, 3970244N

General: Horizontal handle; possible pushed through attachment.

Fabric: SATd Date: 40\% LPrePal, 40\% FPal, 20\% SPal

51 (petrography sample ASP178; 7835-1875-S-7) 707835E, 3971877N

General: Ovoid horizontal handle from a medium to large vessel; pushed through attachment.

Fabric: SATd Date: 40\% LPrePal, 40\% FPal, 20\% SPal

52 (petrography sample ASP179; 9555-0085-S-1) 709557E, 3969757N

General: Thick horizontal handle (round in section) from a large vessel; pushed through attachment.

Fabric: SATd Date: $40 \%$ LPrePal, 40\% FPal, 20\% SPal

53 (petrography sample ASP43; 6335-3475-S-1) 706336E, 3973471N

General: Probably a body fragment with a plastic impressed band on the exterior.

Fabric: SATd Date: 20\% EB2, 20\% LPrePal, 20\% FPal, 20\% SPal, 20\% TPal

54 (petrography sample ASP44; 8045-2205-S-1) 708046E, 3972206N

General: Horned lug and body fragment; lug is made from different clay that fired an entirely different colour.

Fabric: SATd Date: 20\% FN-EB1, 50\% EB2, 10\% LPrePal, 10\% FPal, 10\% SPal

55 (petrography sample ASP22; 3003-15-1-15-1) 706406E, 3973363N

General: Everted rim fragment.

Fabric: SATd Date: 15\% EB2, 15\% LPrePal, 15\% FPal, 15\% SPal, 15\% TPal, 25\%

Post-prehistoric 
56 (petrography sample ASP196; 6555-3265-S-1) 706559E, 3973267N

General: Thick base fragment of a pithos, with rounded edges.

Fabric: SATd Date: 15\% EB2, 15\% LPrePal, 15\% FPal, 15\% SPal, 15\% TPal, 25\%

Post-prehistoric

57 (petrography sample ASP45; 7825-1845-S-3) 707826E, 3971849N

General: Rim sherd with thick ovoid vertical handle (vestigial fake rivet near rim?); possible oval mouthed amphora.

Fabric: SAT(L) Date: 25\% LPrePal, 25\% FPal, 25\% SPal, 25\% TPal

58 (petrography sample ASP80; 2042-11-1-15-1) 707194E, 3972626N

General: Large, ovoid leg of a tripod cooking pot.

Fabric: GROa Date: 20\% EB2, 20\% LPrePal, 20\% FPal, 20\% SPal, 20\% TPal

59 (petrography sample ASP81; 12082-51-1-35-2) 709566E, 3970327N

General: Body fragment from a medium sized vessel, with plastic impressed bands on the exterior.

Fabric: GROa Date: 20\% EB2, 20\% LPrePal, 20\% FPal, 20\% SPal, 20\% TPal (scatter is FPal-SPal)

60 (petrography sample ASP87; 4253-17-1-25-7) 707052E, 3972188N

General: Body fragment (possibly close to the rim) of medium-sized vessel, with plastic impressed band of overlapping discs on exterior.

Fabric: GROa Date: 15\% EB2 15\% LPrePal, 15\% FPal, 40\% SPal, 15\% TPal

61 (petrography sample ASP93; 8425-2065-S-9) 708424E, 3972065N

General: Rim of a medium to large-sized open shape (diameter c. $20 \mathrm{~cm}$ ) with a round in section handle.

Fabric: GROa (bad thin section) Date: $100 \%$ SPal

62 (petrography sample ASP97; 8048-20-1-75-2) 709050E, 3970719N

General: Rim of a large open vessel (basin?) with a plastic impressed band of overlapping discs under rim.

Fabric: GROa Date: 15\% EB2 15\% LPrePal, 15\% FPal, 40\% SPal, 15\% TPal

63 (petrography sample ASP101; 8775-9885-V-1) 708779E, 3969676N

General: Incurved rim fragment with a possible triangular rim lug.

Fabric: GROa Date: 20\% EB2, 20\% LPrePal, 25\% FPal, 25\% SPal, 10\% TPal

64 (petrography sample ASP103; 8815-9915-S-2) 708814E, 3969641N

General: Rim fragment from a jar (diameter $21 \mathrm{~cm}$ ), with a plastic impressed band directly beneath rim.

Fabric: GROa Date: 100\% EB2

65 (petrography sample ASP106; 8425-2065-S-6) 708424E, 3972069N

General: Base (diameter $21 \mathrm{~cm}$ ) with a plastic impressed band and thick, parallel diagonal incised lines along interior base wall

Fabric: GROa Date: 10\% EB2, 10\% LPrePal, 35\% FPal, 35\% SPal, 10\% TPal (scatter is [FPal-]SPal) 
66 (petrography sample ASP112; 12039-57-1-95-2) 709714E, 3970379N

General: Rim of a pithos.

Fabric: GROa Date: $30 \%$ LPrePal, 30\% FPal, 20\% SPal, 20\% TPal (scatter is FPalSPal)

67 (petrography sample ASP175; 4161-29-1-5-1) 706491E, 3972223N

General: Upswung horizontal handle from a medium-sized vessel; round in section.

Fabric: GROa (grog+chert, strange isotropic tcfs in groundmass, inactive; [medium]coarse; refires red; optically inactive) Date: 20\% EB2, 20\% LPrePal, 20\% FPal, $20 \%$ SPal, $20 \%$

68 (petrography sample ASP176; 7081-20-1-65-3) 708993E, 3973095N

General: Horizontal handle from a medium-sized vessel; round in section

Fabric: GROa Date: 20\% EB2, 30\% LPrePal, 30\% FPal, 20\% SPal (scatter is FNEBA)

69 (petrography sample ASP86; 8795-9925-S-5) 708793E, 3969660N

General: Rim fragment with very little curvature. Large, flaring bowl, lid or pan.

Fabric: GROa Date: 20\% EB2, 20\% LPrePal, 20\% FPal, 20\% SPal, 20\% TPal

70 (petrography sample ASP89; 8045-2145-S-2) 708045E, 3972146N

General: Body fragment with rounded plastic band on exterior.

Fabric: GROa Date: 30\% FN-EB1, 30\% EB2, 10\% LPrePal, 10\% FPal, 10\% SPal, $10 \% \mathrm{TPal}$

71 (petrography sample ASP84; 8035-2215-S-1) 708035E, 3972218N

General: Thick vertical handle coming off the rim of large vessel; ovoid in section. Perhaps from a ladle.

Fabric: GROa Date: 30\% EB2, 10\% LPrePal, 10\% FPal, 10\% SPal, 40\% TPal

72 (petrography sample ASP189; 8045-2185-S-1) 708046E, 3972187N

General: Horizontal handle for medium to large sized vessel; round in section, possible pushed through attachment.

Fabric: GROa Date: 20\% EB2, 20\% LPrePal, 20\% FPal, 20\% SPal, 20\% TPal

73 (petrography sample ASP72; 7955-2145-V-2) 707955E, 3972143N

General: Body sherd with plastic band on the exterior.

Fabric: GROb Date: 100\% FN-EB1

74 (petrography sample ASP73; 8045-2205-S-1) 708046E, 3972206N

General: Body sherd with pyramid-shaped lug on exterior; lug made from a different clay which has fired an entirely different colour.

Fabric: GROb Date: 20\% FN-EB1, 50\% EB2, 10\% LPrePal, 10\% FPal, 10\% SPal

75 (petrography sample ASP74; 8035-2155-S-3) 708038E, 3972155N

General: Body sherd with thin plastic bands on the exterior.

Fabric: GROb Date: 70\% FN-EB1, 30\% EB2

76 (petrography sample ASP76; 3645-34-1-35-1) 708082E, 3970822N 
General: Horizontal handle (with crack between body and handle attachment)

Fabric: GROb Date: 70\% FN-EB1, 30\% EB2

77 (petrography sample ASP77; 3470-14-1-5-1) 708052E, 3971402N

General: Full profile of a sauceboat. Incised dashes above exterior base; red-brown slipped and burnished surface.

Fabric: GROb Date: $100 \%$ EB2

78 (petrography sample ASP82; 8025-2205-S-1) 708027E, 3972210N

General: Body sherd with thin plastic impressed band on exterior.

Fabric: GROb Date: 70\% FN-EB1, 30\% EB2

79 (petrography sample ASP85; 8055-2225-S-1) 708052E, 3972224N

General: Possible rim fragment.

Fabric: GROb Date: 70\% FN-EB1, 30\% EB2

80 (petrography sample ASP102; 8815-9885-S-1) 708812E, 3969671N

General: Horizontal handle with diagonal slash marks.

Fabric: GROb Date: 100\% EB2

81 (petrography sample ASP105; 9385-0815-S-2) 709389E, 3970812N

General: Vertical ovoid strap handle.

Fabric: GROb Date: 20\% EB2, 20\% LPrePal, 20\% FPal, 20\% SPal, 20\% TPal

82 (petrography sample ASP107; 9565-0085-S-1) 709566E, 3970090N

General: Horizontal handle, round in section, slightly pushed through, from medium sized vessel (cracked surface appearance).

Fabric: GROb Date: 30\% LPrePal, 30\% FPal, 20\% SPal, 20\% TPal

83 (petrography sample ASP110; 7815-1535-S-1) 707819E, 3971536N

General: Body fragment with plastic impressed band on exterior.

Fabric: GROb Date: 10\% FN-EB1, 30\% EB2, 10\% LPrePal, 10\% FPal, 30\% SPal, $10 \% \mathrm{TPal}$

84 (petrography sample ASP100; 8048-20-1-35-1) 709088E, 3970699N

General: Rim fragment of a jar. Mottled firing.

Fabric: GROc Date: 20\% FPal, 60\% SPal, 20\% TPal

85 (petrography sample ASP113; 6725-1485-S-1) 706720E, 3971482N

General: Rim fragment of a jar with a plastic impressed band below rim.

Fabric: GROc Date: 20\% FPal, 60\% SPal, 20\% TPal

86 (petrography sample ASP118; 6265-2425-S-2) 706266E, 3972421N

General: Base fragment (diameter $15 \mathrm{~cm}$ ) with a plastic impressed band. Overfired.

Fabric: GROc Date: 10\% EB2, 10\% LPrePal, 35\% FPal, 35\% SPal, 10\% TPal (scatter is SPal -TPal)

87 (petrography sample ASP94; 9385-0815-V-16) 709382E, 3970817N

General: Rim fragment with plastic impressed band under rim; mottled grey/pink firing. 
Fabric: GROc Date: 20\% SPal, 80\% TPal

88 (petrography sample ASP116; 7015-2165-S-1) 707018E, 3972165N

General: Horizontal, rolled handle with partial pushed through attachment; hard fired. Fabric: GROc Date: 20\% EB2, 20\% LPrePal, 20\% FPal, 20\% SPal, 20\% TPal

89 (petrography sample ASP95; 8815-1705-S-1) 708813E, 3969661N

General: Shoulder fragment from a jar. Three plastic ribs on neck/body join; black slip on interior neck and between ribs on exterior.

Fabric: GRO(L1) Date: 30\% LPrePal, 30\% FPal, 20\% SPal, 20\% TPal

90 (petrography sample ASP96; 7315-3375-S-2) 707315E, 3973379N

General: Body fragment with an abraded plastic band on the exterior; very hard, mottled firing.

Fabric: GRO(L2) Date: 30\% FPal, 30\% SPal, 40\% TPal

91 (petrography sample ASP99; 12171-51-1-35-4) 709579E, 3970089N

General: Rim of medium to large open vessel with a plastic impressed band on the exterior, just below a plain rim.

Fabric: GRO(L3) Date: 20\% FPal, 80\% SPal

92 (petrography sample ASP111; 9215-1125-S-1) 709219E, 3971123N

General: Vessel part unclear, but probably a medium-sized, rounded handle (or much less likely, a small ovoid leg) with two small indentations at one end.

Fabric: GRO(L4) Date: 30\% FN-EB1, 30\% EB2, 10\% LPrePal, 10\% FPal, 10\% SPal, $10 \%$ TPal (scatter is FN-EB1)

93 (petrography sample ASP115; 9385-0875-S-1) 709388E, 3970880N

General: Rim fragment of a jar with a handle scar. The handle is round in section and directly under the rim.

Fabric: GRO(L5) Date: 10\% LPrePal, 10\% FPal, 10\% SPal, 70\% TPal

94 (petrography sample ASP88; 8015-2195-V-1) 708014E, 3972194N

General: Fragment of unknown vessel part, with incision, possibly a deeply incised handle.

Fabric: GRO(L6) Date: 30\% FN-EB1, 30\% EB2, 10\% LPrePal, 10\% FPal, 10\% SPal, $10 \%$ TPal

95 (petrography sample ASP140; 8035-2215-V-1) 708031E, 3972211N

General: Body fragment with a plastic impressed and differentially tempered band on the exterior; very hard fired.

Fabric: GRO(L7) Date: 20\% EB2, 20\% LPrePal, 20\% FPal, 20\% SPal, 20\% TPal

96 (petrography sample ASP120; 5028-4-1-15-1) 707936E, 3973398N

General: Rim and horizontal handle from a large open shape, possibly a basin.

Fabric: MUTa Date: 5\% EB2, 5\% LPrePal, 35\% FPal, 35\% SPal, 20\% TPal

97 (petrography sample ASP123; 8311-14-1-95-1) 709550E, 3970689N

General: Medium ovoid leg from a tripod cooking pot.

Fabric: MUTa Date: 10\% EB2, 10\% LPrePal, 35\% FPal, 35\% SPal, 10\% TPal 
98 (petrography sample ASP124; 3741-19-1-75-1) 708299E, 3969711N

General: Rim fragment of a jar (diameter uncertain but large).

Fabric: MUTa Date: 40\% FPal, 40\% SPal, 20\% TPal

99 (petrography sample ASP125; 3566-42-1-5-1) 706567E, 3971596N

General: Pithos rim (diameter uncertain but large).

Fabric: MUTa Date: 15\% EB2, 15\% LPrePal, 15\% FPal, 15\% SPal, 15\% TPal, 25\%

Post-prehistoric

100 (petrography sample ASP128; 8015-2145-S-1) 708012E, 3972143N

General: Two joining body fragments; plastic impressed band next to vertical handle.

Fabric: MUTa Date: 10\% EB2, 10\% LPrePal, 35\% FPal, 35\% SPal, 10\% TPal

101 (petrography sample ASP132; 6715-1495-V-1) 706719E, 3971490N

General: Body fragment with a plastic impressed band on the exterior.

Fabric: MUTa Date: 50\% SPal, 40\% TPal, 10\% PostPal

102 (petrography sample ASP133; 7305-3405-S-1) 707301E, 3973406N

General: Horizontal handle from medium sized vessel; rounded ovoid in section.

Fabric: MUTa Date: 10\% EB2, 10\% LPrePal, 35\% FPal, 35\% SPal, 10\% TPal

103 (petrography sample ASP139; 8415-2095-S-1) 708414E, 3972094N

General: Pithos rim.

Fabric: MUTa Date: $100 \%$ SPal (scatter is [FPal-]SPal)

104 (petrography sample ASP126; 11062-45-1-5-1) 707646E, 3971526N

General: Probably a rim fragment from a jar (diameter $>30 \mathrm{~cm}$ ).

Fabric: MUTb (mudstone/siltstone, few chert (radiolarian), acid tuff?; coarse; refires red; optically moderately to slightly active) Date: 10\% LPrePal, 40\% FPal, 40\% SPal, $10 \%$ TPal (scatter is [FPal-]SPal)

Parallels:

105 (petrography sample ASP129; 8125-2365-V-1) 708124E, 3972369N

General: Large body sherd with a plastic impressed band in a different clay on the exterior.

Fabric: MUTb Date: 50\% SPal, 40\% TPal, 10\% PostPal (scatter is [FPal-]SPal)

106 (petrography sample ASP130; 8825-9925-S-2) 708826E, 3969652N

General: Body fragment of a jar with an abraded rib.

Fabric: MUTb Date: 20\% EB2\%, 20\% LPrePal, 20\% FPal, 20\% SPal, 20\% TPal

107 (petrography sample ASP138; 9645-0375-S-4) 709645E, 3970376N

General: Large ovoid leg of a tripod cooking pot.

Fabric: MUTb Date: 10\% EB2, 10\% LPrePal, 30\% FPal, 30\% SPal, 20\% TPal

108 (petrography sample ASP141; 8005-2155-V-1) 708005E, 3972158N

General: Probable base fragment of a pithos with raised band around the exterior base.

Fabric: MUTc Date: 20\% FPal, 60\% SPal, 20\% TPal 
109 (petrography sample ASP142; 6315-3465-S-1) 706317E, 3973462N

General: Horizontal ovoid handle from medium sized vessel.

Fabric: MUTc Date: $40 \%$ FPal, 40\% SPal, 20\% TPal

110 (petrography sample ASP144; 7315-3405-S-2) 707314E, 3973402N

General: Thin horizontal handle; round in section.

Fabric: MUTc Date: 40\% FPal, 40\% SPal, 20\% TPal

111 (petrography sample ASP145; 7305-3425-S-3) 707302E, 3973425N

General: Ovoid handle.

Fabric: MUTc Date: $40 \%$ FPal, 40\% SPal, 20\% TPal

112 (petrography sample ASP146; 2006-2-1-5-2) 707291E, 3973416N

General: Base fragment of a medium sized, probably closed, vessel (diameter $11 \mathrm{~cm}$ ).

Fabric: MUT(L1) Date: 40\% FPal, 40\% SPal, 20\% TPal

113 (petrography sample ASP135; 7325-3395-S-2) 707330E, 3973398N

General: Poorly preserved and shape unclear. Could be a jar rim, the beginnings of a thin ovoid strap handle or perhaps a ring base. Differentially tempered.

Fabric: MUT(L2) Date: 20\% EB2, 20\% LPrePal, 20\% FPal, 20\% SPal 20\% TPal

114 (petrography sample ASP131; 7295-3405-S-3) 707292E, 3973400N

General: Very large, ovoid leg of a tripod cooking pot.

Fabric: MUT(L3) Date: 10\% EB2, 10\% LPrePal, 35\% FPal, 35\% SPal 10\% TPal

115 (petrography sample ASP49; 6555-3265-S-2) 706555E, 3973268N

General: Flaring rim fragment from a bowl, jar or lid.

Fabric: CALa Date: 40\% EB2, 30\% LPrePal, 30\% FPal

116 (petrography sample ASP50; 8005-2155-S-1) 708009E, 3972158N

General: Medium thin ovoid leg from a tripod cooking pot.

Fabric: CALa Date: 30\% EB2, 30\% LPrePal, 30\% FPal, 5\% SPal, 5\% TPal

117 (petrography sample ASP51; 8075-2155-V-2) 708080E, 3972152N

General: Medium ovoid leg from a tripod cooking pot.

Fabric: CALa Date: 30\% EB2, 30\% LPrePal, 30\% FPal, 5\% SPal, 5\% TPal

118 (petrography sample ASP52; 8065-2195-S-2) 708069E, 3972196N

General: Slightly planoconvex, with little curvature. Could be the tip of straight handle but more likely the tip of a tripod cooking pot leg.

Fabric: CALa Date: 30\% EB2, 30\% LPrePal, 30\% FPal, 5\% SPal, 5\% TPal

119 (petrography sample ASP53; 7955-2155-V-2) 707956E, 3972155N

General: Rim fragment of a large open shape with slipped exterior and an incised line on interior under rim.

Fabric: CALa Date: $100 \%$ FN-EB1

120 (petrography sample ASP57; 4222-17-1-15-1) 706797E, 3972226N

General: Medium sized, ovoid leg of a tripod cooking pot. 
Fabric: CALa Date: 30\% EB2, 30\% LPrePal, 30\% FPal, 5\% SPal, 5\% TPal (scatter is FPal-SPal)

121 (petrography sample ASP62; 8015-2175-S-1) 708017E, 3972179N

General: Small thin ovoid leg of a tripod cooking pot.

Fabric: CALa Date: 30\% EB2, 30\% LPrePal, 30\% FPal, 5\% SPal, 5\% TPal

122 (petrography sample ASP70; 8785-9915-S-1) 708783E, 3969666N

General: Medium sized thin ovoid leg of a tripod cooking pot.

Fabric: CALa Date: 30\% EB2, 30\% LPrePal, 30\% FPal, 5\% SPal, 5\% TPal

123 (petrography sample ASP54; 8035-2145-S-1) 708033E, 3972150N

General: Small, rounded leg of a tripod cooking pot.

Fabric: CALb Date: 30\% EB2, 30\% LPrePal, 30\% FPal, 5\% SPal, 5\% TPal

124 (petrography sample ASP60; 3461-19-1-25-1) 707832E, 3971881N

General: Small, ovoid leg of a tripod cooking pot.

Fabric: CALb Date: 30\% EB2, 30\% LPrePal, 30\% FPal, 5\% SPal, 5\% TPal

125 (petrography sample ASP63, 7945-2145-S-2) 707941E, 3972144N

General: Small ovoid leg from a tripod cooking pot with slight ridge on interior.

Fabric: CALb Date: 30\% EB2, 30\% LPrePal, 30\% FPal, 5\% SPal, 5\% TPal

126 (petrography sample ASP69; 7725-1975-V-1) 707727E, 3971980N

General: Large, flaring rim from bowl or pan with faint, diagonal incised lines on rim interior.

Fabric: CALb Date: 60\% EB2, 20\% LPrePal, 20\% FPal (scatter is mainly EB2)

127 (petrography sample ASP71; 6795-2215-S-1) 706796E, 3972214N

General: Possibly a medium, thin ovoid leg from a cooking pot.

Fabric: CALb Date: 30\% EB2, 30\% LPrePal, 30\% FPal, 5\% SPal, 5\% TPal (scatter is FPal-SPal)

128 (petrography sample ASP65; 3429-5-1-15-1) 707959E, 3972157N

General: Medium-sized leg from a tripod cooking pot; ovoid in section.

Fabric: CALb Date: 30\% EB2, 30\% LPrePal, 30\% FPal, 5\% SPal, 5\% TPal

129 (petrography sample ASP55; 8055-2205-S-2) 708051E, 3972205N

General: Medium sized ovoid leg from a tripod cooking pot.

Fabric: CALc Date: 30\% EB2, 30\% LPrePal, 30\% FPal, 5\% SPal, 5\% TPal

130 (petrography samples ASP58 and ASP61; 6555-3255-S-3 and 4) 706558E, 3973253N

General: Almost the full profile of a tripod cooking pot including rim, vertical handle (round in section) and a medium-sized, thin ovoid leg with a ridge on the exterior. Two non-joining fragment but found together at the base of a standing section and very likely to be the same vessel.

Fabric: CALc Date: 20\% EB2, 30\% LPrePal, 40\% FPal, 5\% SPal, 5\% TPal

131 (petrography sample ASP59; 8095-1685-S-2) 708094E, 3971684N 
General: Rim fragment, probably from a deep incurved bowl (or less likely from a large, spouted flaring vessel).

Fabric: CALc Date: 20\% MN-LN, 35\% FN-EB1, 15\% EB2, 15\% LPrePal, $15 \%$

FPal

132 (petrography sample ASP64; 8055-2205-S-1) 708058E, 3972209N

General: Medium sized ovoid leg from a tripod cooking pot.

Fabric: CALc Date: 30\% EB2, 30\% LPrePal, 30\% FPal, 5\% SPal, 5\% TPal

133 (petrography sample ASP67; 8015-2185-V-2) 708011E, 3972184N

General: Horizontal, rounded handle tapering to a thin tip

Fabric: CALc Date: 30\% EB2, 30\% LPrePal, 30\% FPal, 5\% SPal, 5\% TPal

134 (petrography sample ASP68; 8035-2155-S-2) 708033E, 3972158N

General: Shape very unclear, but possibly a handle with sub-rectangular section.

Fabric: CALc Date: 30\% EB2, 30\% LPrePal, 30\% FPal, 5\% SPal, 5\% TPal

135 (petrography sample ASP56; 6755-1475-S-10) 706756E, 3971475N

General: Vertical handle from medium sized vessel; ovoid in section, with a deep incision nearly splitting the handle. Probably from a sauceboat or askos.

Fabric: CAL(L) Date: 100\% EB2

136 (petrography sample ASP155; 2006-2-1-5-1) 707292E, 3973416N

General: Probably a medium-sized leg from a tripod cooking pot; ovoid section

Fabric: SHAa Date: 20\% EB2, 20\% LPrePal, 20\% FPal, 20\% SPal, 20\% TPal

137 (petrography sample ASP158; 3235-19-1-75-1) 705533E, 3974755N

General: Probably a vertical handle but possibly a tripod cooking pot leg.

Fabric: SHAa Date: 20\% EB2, 20\% LPrePal, 20\% FPal, 20\% SPal, 20\% TPal

138 (petrography sample ASP156; 5036-7-1-55-1) 707896E, 3973528N

General: Vertical strap handle; ovoid in section and possibly crested.

Fabric: SHAb Date: 35\% LPrePal, 25\% FPal, 20\% SPal, 20\% TPal

139 (petrography sample ASP157; 4036-14-1-35-1) 706623E, 3972857N

General: Vertical strap handle; ovoid in section, possibly from an oval mouthed amphora.

Fabric: SHAb Date: 25\% LPrePal, 25\% FPal, 25\% SPal, 25\% TPal (scatter is TPal)

140 (petrography sample ASP161; 6815-2255-S-1) 706810E, 3972256N

General: Probable base fragment from a medium to large vessel.

Fabric: SHAb Date: 25\% LPrePal, 25\% FPal, 25\% SPal, 25\% TPal (scatter is FPalSPal)

141 (petrography sample ASP152; 8785-9915-S-4) 708789E, 3969677N

General: Body fragment from a large vessel with a possible plastic band on the exterior.

Fabric: SHAc Date: 15\% EB2, 15\% LPrePal, 15\% FPal, 15\% SPal, 15\% TPal, 25\% Post-prehistoric 
142 (petrography sample ASP159; 3457-19-1-25-1) 708038E, 3971816N

General: Rounded ovoid handle from medium to large vessel.

Fabric: SHAc Date: 20\% EB2, 20\% LPrePal, 20\% FPal, 20\% SPal, 20\% TPal

143 (petrography sample ASP150; 4029-14-1-15-1) 706357E, 3973141N

General: Rim of a jar (diameter ca. $25 \mathrm{~cm}$ ).

Fabric: SHAd Date: 20\% LPrePal, 20\% FPal, 20\% SPal, 20\% TPal, 20\% Postprehistoric

144 (petrography sample ASP153; 6545-3245-S-1) 706547E, 3973249N

General: Thick vertical handle from large vessel; rounded ovoid in section.

Fabric: SHAd Date: 15\% LPrePal, 15\% FPal, 15\% SPal, 15\% TPal, 40\% Postprehistoric

145 (petrography sample ASP149; 8078-27-1-95-1) 708777E, 3971860N

General: Rim fragment, possibly from a deep bowl.

Fabric: SHAe Date: 30\% EB2, 5\% LPrePal, 5\% FPal, 5\% SPal, 5\% TPal, 50\% Postprehistoric.

146 (petrography sample ASP160; 9395-0895-S-2) 709394E, 3970891N

General: Vertical strap handle from a medium-sized closed jar; highly differential tempering between body and handle.

Fabric: SHAe Date: 20\% LPrePal, 20\% FPal, 20\% SPal, 20\% TPal, 20\% Postprehistoric

147 (petrography sample ASP172; 3623-2-1-55-1) 708433E, 3970227N

General: Large, tapering horizontal handle from a jar.

Fabric: SHAe Date: 20\% EB2, 20\% LPrePal, 20\% FPal, 20\% SPal, 20\% TPal (scatter is FN-EBA)

148 (petrography sample ASP151; 8184-14-1-25-1) 709372E, 3970881N

General: Rounded ovoid handle from medium sized vessel.

Fabric: SHA(L) Date: 20\% EB2, 20\% LPrePal, 20\% FPal, 20\% SPal, 20\% TPal

149 (petrography sample ASP154; 7325-3415-S-1) 707326E, 3973412N

General: Horizontal handle from a large vessels, probably a pithos.

Fabric: SHA(L) Date: 20\% EB2, 20\% LPrePal, 20\% FPal, 20\% SPal, 20\% TPal

150 (petrography sample ASP148; 11031-5-1-15-1) 707358E, 3972096N

General: Rim fragment from a jar.

Fabric: SHA(L) Date: 10\% EB2, 10\% LPrePal, 10\% FPal, 10\% SPal, 10\% TPal, $50 \%$ Post-prehistoric

151 (petrography sample ASP165; 16013-35-1-85-1) 707643E, 3969603N

General: Rim fragment of a pithos - likely to be later in date.

Fabric: SHA(L) Date: 20\% SPal, 20\% TPal, 60\% Post-prehistoric

152 (petrography sample ASP147; 6084-27-1-55-1) 708465E, 3970763N 
General: Horizontal strap handle below the thickened and everted rim of a large open vessel.

Fabric: SHA(L) Date: 15\% LPrePal, 15\% FPal, 40\% SPal, 15\% TPal, 15\% Postprehistoric

153 (petrography sample ASP162; 12171-51-1-35-3) 709574E, 3970088N

General: Vertical handle from a closed jar (possibly an oval mouthed amphora) or perhaps a ladle.

Fabric: MIC Date: $70 \%$ SPal, 30\% TPal

154 (petrography sample ASP163; 4035-14-1-45-2) 706569E, 3972813N

General: Rim or pedestal base fragment from a pithos.

Fabric: MIC Date: $70 \%$ SPal, $30 \%$ TPal (scatter is [FPal-]SPal)

155 (petrography sample ASP164; 11085-4-1-55-1) 707805E, 3971553N

General: Rim fragment from a jar (diameter $25-30 \mathrm{~cm}$ ).

Fabric: MIC Date: $100 \%$ SPal (scatter is [FPal-]SPal)

156 (petrography sample ASP166; 1064-2-1-5-2) 707128E, 3973164N

General: Medium-sized leg from a tripod cooking pot; ovoid in section.

Fabric: MIC Date: $80 \%$ SPal, $20 \%$ TPal

157 (petrography sample ASP167; 7149-27-1-55-1) 708130E, 3972334N

General: Medium-sized leg from a tripod cooking pot; ovoid in section.

Fabric: MIC Date: $80 \%$ SPal, $20 \%$ TPal (scatter is [FPal-]SPal)

158 (petrography sample ASP168; 9078-32-1-5-1) 708791E, 3969913N

General: Medium-sized leg from a tripod cooking pot; ovoid in section.

Fabric: MIC Date: $80 \%$ SPal, 20\% TPal

159 (petrography sample ASP169; 11089-4-1-65-1) 707796E, 3971534N

General: Body fragment with narrow ribbing on exterior.

Fabric: MIC Date: $100 \%$ SPal (scatter is [FPal-]SPal)

160 (petrography sample ASP170; 6715-1485-V-1) 706716E, 3971481N

General: Body fragment with a horizontally pattern-burnished exterior.

Fabric: MIC Date: 40\% EB2, 40\% SPal, 20\% TPal

161 (petrography sample ASP173; 10001-44-1-5-1) 706767E, 3971459N

General: Horizontal handle from a medium to large-sized vessel.

Fabric: MIC Date: $70 \%$ EB2, 30\% SPal

162 (petrography sample ASP174; 4019-10-1-15-2) 706627E, 3972901N

General: Handle from a medium to large-sized vessel.

Fabric: MIC Date: 20\% EB2, 40\% SPal, 40\% TPal (scatter is TPal)

163 (petrography sample ASP171; 4174-14-1-45-1) 706705E, 3971879N

General: Horizontal handle with incised lines at the base; from a medium to large jar.

Fabric: MIC(L2) Date: $80 \%$ EB2, 10\% SPal, 10\% TPal 
164 (petrography sample ASP83; 8105-1685-V-1) 708103E, 3971687N

General: Rim fragment of a bowl.

Fabric: CAS Date: 60\% EB2, 10\% LPrePal, 10\% FPal, 10\% SPal, 10\% TPal

165 (petrography sample ASP188; 6635-2925-V-2) 706632E, 3972925N

General: Vertical handle (less likely, a leg) made up of three adjacent rods, probably from an ampharoid krater

Fabric: CAS Date: $100 \%$ TPal

166 (petrography sample ASP79; 4247-39-1-65-1) 707094E, 3972016N

General: Small, ovoid leg from a tripod cooking pot.

Fabric: OUT Date: 20\% EB2, 20\% LPrePal, 20\% FPal, 20\% SPal, 20\% TPal

167 (petrography sample ASP109; 9825-8695-S-1) 709823E, 3968692N

General: Rim and vertical handle from a ladle; handle is round in section.

Fabric: OUT Date: $70 \%$ FN-EB1, 30\% TPal (scatter is FN-EBA)

168 (petrography sample ASP190; 3422-5-1-15-1) 707774E, 3972162N

General: Base and stem fragment from a kylix. Stem is partly or wholly hollow.

Fabric: OUT Date: $100 \%$ TPal

169 (petrography sample ASP191; 4020-10-1-5-1) 706665E, 3972748N

General: Base and foot fragment from a small open vessel (goblet?).

Fabric: OUT Date: 15\% EB2, 40\% LPrePal, 15\% FPal, 15\% SPal, 15\% TPal

170 (petrography sample ASP193; 4210-14-1-45-1) 706688E, 3972315N

General: Rim fragment (diameter 9-10 cm) from a jar, with handle scar below rim. Over-fired and likely to be later in date.

Fabric: OUT Date: 30\% EB2, 70\% Post-prehistoric (the prehistoric part of this scatter is FPal-SPal)

171 (petrography sample ASP194; 8104-36-1-5-1) 709301E, 3971547N

General: Probably a large vertical handle (and later in date) or possibly a mediumsized ovoid leg from a tripod cooking pot.

Fabric: OUT Date: 10\% EB2, 10\% LPrePal, 10\% FPal, 10\% SPal, 10\% TPal, 50\% Post-prehistoric

172 (petrography sample ASP195; 6325-3425-S-4) 706324E, 3973422N

General: Stem fragment from a kylix

Fabric: OUT Date: $100 \%$ TPal

173 (petrography sample ASP198; 6755-1475-S-13) 706756E, 3971472N

General: Rim fragment from a very shallow vessel (diameter ca. $27 \mathrm{~cm}$ ).

Fabric: OUT Date: 50\% EB2, 20\% LPrePal, 10\% FPal, 10\% SPal, 10\% TPal

174 (petrography sample ASP200; 7945-3425-V-16) 707945E, 3973426N

General: Vertical handle fragment with a deep incision down the centre and possible red slip on the interior of the incision. Probably from a sauceboat or askos.

Fabric: OUT Date: $100 \%$ EB2 
175 (7131-40-1-5-1) 708210E, 3972054N

General: Rim fragment from a 'cheesepot', with a burnished surface and two prefiring holes near rim.

Fabric: GRO (macroscopic resemblance to GROb) Date: $100 \%$ FN-EB1

176 (9305-1535-S-1) 709304E, 3971537N

General: Thick disc base.

Fabric: GRO (macroscopic resemblance to GROb) Date: 100\% FN-EB1

177 (8055-20-1-5-3) 709198E, 3970907N

General: Body fragment with impressed herringbone decoration

Fabric: MIC Date: $100 \%$ EB2

178 (7835-1885-S-1) 707833E, 3971885N

General: Rim fragment (diameter c.20-25 cm) with possible diagonal striations on the exterior or heavy burnishing. Probably from a medium-coarse sauceboat.

Fabric: MIC Date: $80 \%$ EB2, 20\% SPal

179 (7795-2145-V-3) 707794E, 3972144N

General: Body fragment with two impressed plastic bands on the exterior, one with chevrons and the other with diagonal lines. Very little curvature.

Fabric: GRO (sub-group uncertain) Date: 100\% EB2

180 (3422-16-1-55-1) 707805E, 3972184N

General: Vertical handle from a medium-sized closed jar; possibly crested.

Fabric: GRO (sub-group uncertain) Date: 10\% EB2, 50\% LPrepal, 20\% FPal, 10\% SPal, $10 \%$ TPal

181 (6755-1475-V-7) 706752E, 3971470N

General: Carinated body fragment from a cup with black slip on both the interior and exterior. Probably from a carinated cup or less likely the bulge from Vapheio cup.

Fabric: Fine (fabric uncertain) Date: $80 \% \mathrm{FPal}, 20 \% \mathrm{SPal}$

182 (6755-1485-S-2) 706756E, 3971480

General: Rim fragment of a medium sized jar, with plastic band of overlapping discs below rim.

Fabric: GRO (sub-group uncertain) Date: $100 \%$ SPal

183 (6705-1475-S-1) 706703E, 3971471N

General: Rim fragment of a large open shape with a plastic band of overlapping discs directly below the rim.

Fabric: GRO (sub-group uncertain) Date: $100 \%$ SPal

184 (8150-29-1-5-4) 706635E, 3973281N

General: Kylix stem with pierced pre-firing hole through stem

Fabric: Fine (fabric uncertain, but a silty clay with argillaceous fragments) Date: $100 \%$ TPal

185(9215-0585-V-1) 709211E, 3970587N 
General: Kylix stem with a pre-firing hole just below the bowl/stem join.

Fabric: Fine (fabric uncertain, but a silty clay with argillaceous fragments) Date: $80 \%$ TPal, $20 \%$ Postpal 


\section{FIGURE CAPTIONS}

1. Two maps of Antikythera showing the distribution of a) all definite and possible prehistoric sherds from first stage surface collection during fieldwalking (coverage was island-wide in lines $15 \mathrm{~m}$ apart), and b) the location of the grids (i.e. groups of $10 \times 10 \mathrm{~m}$ squares) used in second stage collection. Gridlines for the UTM (zone 34N, WGS84) coordinate system used in the catalogue are also shown.

2. Line drawings and photographs of SATa samples

3. Line drawings and photographs of SATa samples (continued)

4. Line drawings and photographs of SATa samples (continued)

5. Line drawings and photographs of SATb samples

6. Line drawings and photographs of SATb samples (continued)

7. Line drawings and photographs of SATc samples

8. Line drawings and photographs of SATd and SAT loner samples

9. Line drawings and photographs of GROa samples

10. Line drawings and photographs of GROa samples (continued)

11. Line drawings and photographs of GROb samples

12. Line drawings and photographs of GROc samples

13. Line drawings and photographs of GRO loner samples

14. Line drawings and photographs of MUTa samples

15. Line drawings and photographs of MUTb samples

16. Line drawings and photographs of MUTc and MUT loner samples

17. Line drawings and photographs of CALa and $b$ samples

18. Line drawings and photographs of CALc and CAL loner samples

19. Line drawings and photographs of SHAa, b, c and d samples

20. Line drawings and photographs of SHAe and SHA loner samples

21. Line drawings and photographs of MIC samples

22. Line drawings and photographs of CAS samples as well as other outlying fabrics

23. Line drawings and photographs of other, unsampled but catalogued sherds that are mentioned in the text. 


\section{COLOUR PLATE}

1. Petrographic thin sections: a) SATa (8, ASP15), b) SATb (26, ASP4), c) SATc (45, ASP21), d) SATd (51, ASP178, e) GROa (66, ASP112), f) GROb (80, ASP102), g) GROc (85, ASP113), h) MUTa (101, ASP132), i) MUTb (105, ASP129), j) MUTc (108, ASP141), k) CALa (117, ASP51), 1) CALb (124, ASP60), m) CALc (130, ASP58), n) SHAa (136, ASP155), o) SHAd (144, ASP153), p) SHAe (147, ASP172), q) MIC (155, ASP164), r) CAS (165, ASP188). Thin sections of SHAb and SHAc have not been shown here. All thin section photographs were taken under crossed polars, width of field $5.9 \mathrm{~mm}$. 


\section{BIBLIOGRAPHY}

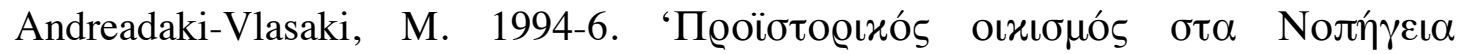

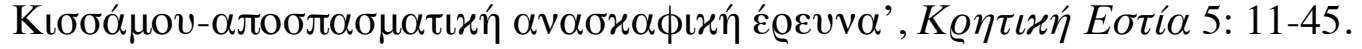

Andreadaki-Vlasaki, M. 1997. 'Craftsmanship at MM Khamalevri in Rethymnon', in Laffineur, R. and Betancourt, P. P. (eds) Techne. Craftsmen, Craftsmwomen and Craftmanship in the Aegean Bronze Age: 37-44. Liège: University of Liège.

Bevan, A., Conolly, J. and Tsaravopoulos, A. 2008. 'The Fragile Communities of Antikythera', Archaeology International 10: 32-36.

Bevan, A. and Conolly, J. in prep. The Fragile Communities of Antikythera (book in preparation, title is provisional).

Blegen, C. W. and Rawson, M. 1966 The Palace of Nestor at Pylos in Western Messenia (Vol.1), Princeton: Princeton University Press.

Broodbank, C. 1999. 'Kythera Survey: Preliminary Report on the 1998 Season', Annual of the British School at Athens 94: 191-214.

Broodbank, C. and Kiriatzi, E. 2007. 'The first 'Minoans' of Kythera revisited: Technology, demography, and landscape in the Prepalatial Aegean', American Journal of Archaeology 111:241-274.

Broodbank, C., Kiriatzi, E. and J.B. Rutter, 2005 'From pharaoh's feet to the slavewomen of Pylos? The history and cultural dynamics of Kythera in the Third Palace period.' In A. Dakouri-Hild and S.E. Sheratt (eds.), Autochthon. Papers Presented to O.T.P.K. Dickinson on the Occasion of his Retirement: 70-96. BAR International Series 1432, Oxford: Archaeopress.

Bullock, P., Federoff, N., Jongerius, A., Stoops, G. and T. Tursina 1985. Handbook for Soil Thin Section Description. Wolverhampton: Wayne Research.

Chandler, G.M. 2001. Comparative Petrographic Analysis of Sherds from five Minoan Sites in Western Crete. In Y. Bassiakos, E. Aloupi and Y. Facorellis (eds) Archaeometry Issues in Greek Prehistory and Antiquity: 379-396. Athens: Hellenic Society of Archaeometry and Society of Messenian Archaeological Studies.

Christakis, K.S. 2005. Cretan Bronze Age Pithoi. Traditions and Trends in the Production and Consumption of Storage Containers in Bronze Age Crete. Philadelphia: INSTAP Academic Press. 
Day, P., Joyner, L., Kiriatzi, E. and M. Relaki. 2005. Petrographic Analysis of some FN-EMII Pottery from the Kavousi Area. In G. Gessell and L. Day (eds) Kavousi I: The Archaeological Survey of the Kavousi Region. Philadelphia: INSTAP Academic Press

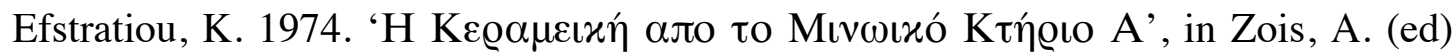

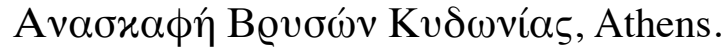

Galeos, A. and E. Drandaki 1993. Geological Map of Greece, Antikythera Island Sheet, scale 1:50.000. Athens: Institute of Geology and Mineral Exploration.

Hallager, B.P. 2000. 'The Late Minoan IIIC pottery, in Hallager, E. and Hallager, B. P., (eds), The Greek Swedish Excavations at the Agia Aikaterini Square Kastel li, Khania 1970-1987, volume 2, pages 135-174. Paul Astroms, Stockholm.

Hallager, B.P. 2003. 'The Late Minoan IIIb:2 Pottery', in Hallager, E. and Hallager, B. P. (eds), The Greek Swedish Excavations at the Agia Aikaterini Square Kastelli, Khania 1970-1987 and 2001, volume 3, pages 197-265. Paul Astroms, Savedalen.

Kanta, A. and Rocchetti, L. 1989. 'La ceramica del primo edificio', in Tzedakis, Y. and Sacconi, A. (eds) Scavi a Nerokourou, Kydonias (Richerche Greco-Italiane in Creta Occidentale I): 103-273. Rome: Incunabula Graeca.

Kanta, A. 1980. The Late Minoan III Period in Crete. A Survey of Sites, Pottery and their Distribution. Number 58 in Studies in Mediterranean Archaeology. Paul Astroms, Goteborg.

Karantzali, E. 1996. Le Bronze Ancien dans les Cyclades et en Crète. Les Relations entre les Deux Régions Influence de la Grèce Continentale, Oxford: British Archaeological Reports International.

Kiriatzi, E. 2003. Sherds, Fabrics and Clay Sources: Reconstructing the Ceramic Landscapes of Prehistoric Kythera. In K. P. Foster and R. Laffineur (eds) Metron: Measuring the Aegean Bronze Age (Aegaeum 24): 123 130. Liège: Université de Liège.

Melas, M. 1999. 'The ethnography of Minoan and Mycenaean beekeeping' in Betancourt, P. P., Karageorghis, V., Laffineur, R., and Nieimeier, W.-D., (eds), Meletemata. Studies in Aegean Archaeology Presented to Malcolm Wiener, volume 2, pages 485-491. Universite de Liege, Liege. 


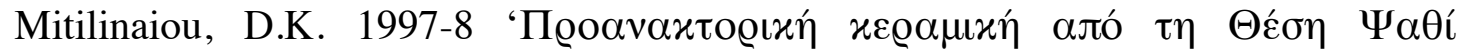

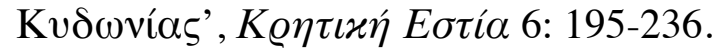

Moody, J., Nixon, L., Price, S., and Rackham, O. 2000. The Sphakia Survey: Internet Edition.

Moody, J.A. 1985. 'The development of a Bronze Age coarse ware chronology for the Khania area of west Crete'. Temple University Aegean Symposium 10: 51-65.

Moody, J. 1987. 'The environmental and cultural prehistory of the Khania region of West Crete: neolithic through late Bronze Age'. Unpublished PhD dissertation, University of Minnesota.

Moody, J. 2004. 'Western Crete in the Bronze Age: A Survey of the Evidence'. In Day, L., Mook, M. and J. Muhly (eds). Crete beyond the palaces: Proceedings of the Crete 2000 conference. INSTAP Academic Press. Philadelphia, PA.

Moody, J. 2005. 'Preliminary report, Vrokastro Survey Pottery Fabrics.' In B. Hayden (ed), Reports on the Vrokastro area, Eastern Crete, volume 3: The Vrokastro Regional Survey project: sites and pottery. University of Pennsylvania, Philadelphia, PA.

Moody, J.A., Lewis, H., Robinson, J. and Nixon, L. 2003. 'Ceramic fabric analysis and survey archaeology: the Sphakia survey', Annual of the British School at Athens 98: $37-105$.

Mook. M. 2005. 'The Kavousi Fabrics: A Typology for the Coarse Pottery of the Mirabello Region of East Crete'. In G. Gessell and L. Day (eds). Kavousi i: the archaeological survey of the Kavousi region. INSTAP Academic Press. Philadelphia, PA.

Nodarou, E. 2003. Pottery Production, Distribution and Consumption in Early Minoan West Crete: An Analytical Perspective (PhD thesis, University of Sheffield).

Nowicki, K. 2000. Defensible Sites in Crete c.1200 - 800 B.C. (LM IIIB/IIIC through Early Geometric), Liège: University of Liège (Aegaeum 21).

Rice, P.M. 1987. Pottery Analysis: A Sourcebook. Chicago: University of Chicago Press. 
Tzedakis, Y. 1969. L'atelier de ceramique postpalatial a Kydonia. Bulletin de Correspondences Helleniques, 93:396-418.

Vagnetti, L.A. 1989. Saggi negli strati neolitici. In Tzedakis, Y. and Sacconi, A., (eds) Scavi a Nerokourou, Kydonias (Richerche Greco-Italiane in Creta Occidentale I): 11100. Rome: Edizione dell' Ateneo.

Warren, P. and J. Tzedakis. 1974. Debla. An Early Minoan Settlement in Western Crete. Annual of the British School at Athens 69: 299-342.

Whitbread, I.K. 1986. The Characterisation of Argillaceous Inclusions in Ceramic Thin Sections. Archaeometry 28: 79-88.

Whitbread, I.K. 1989. A Proposal for the Systematic Description of Thin Sections. Towards the Study of Ancient Ceramic Technology. In Y. Maniatis (ed.) Archaeometry: Proceedings of the 25th International Symposium: 127-138. Amsterdam: Elsevier.

Whitbread, I.K. 1995. 'Greek Transport Amphorae. A Petrological and Archaeological Study', Fitch Laboratory Occasional Paper 4. Athens: British School at Athens.

Wilson, D.E. and P.M. Day 1994. Ceramic Regionalism in Prepalatial Central Crete: The Messara Imports at EM I to EM IIA Knossos. Annual of the British School at Athens 89: 1-87. 


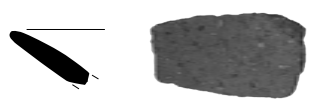

1 (ASP1; 2021-8-1-95-1)
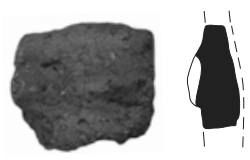

2 (ASP2; 5028-2-1-15)

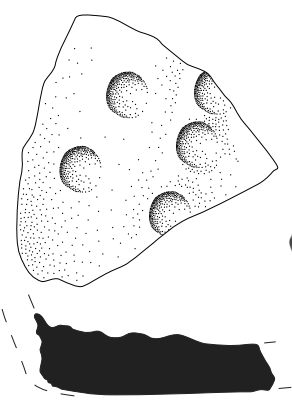

3 (ASP3; 10028-42-1-45-3)

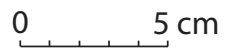

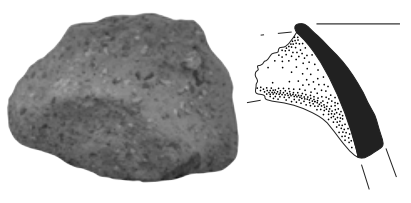

5 (ASP7; 3449-12-1-25-1)

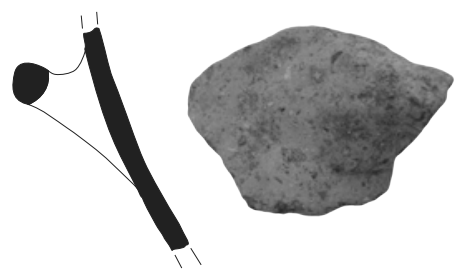

6 (ASP9; 8425-2105-S-4)
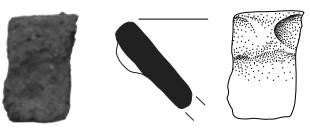

7 (ASP11; 6795-2245-S-2)
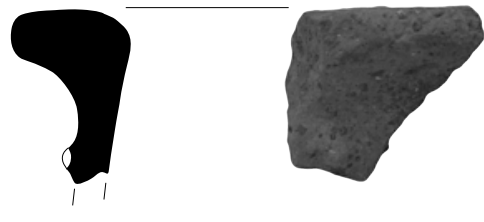

4 (ASP6; 9034-29-1-75-1)

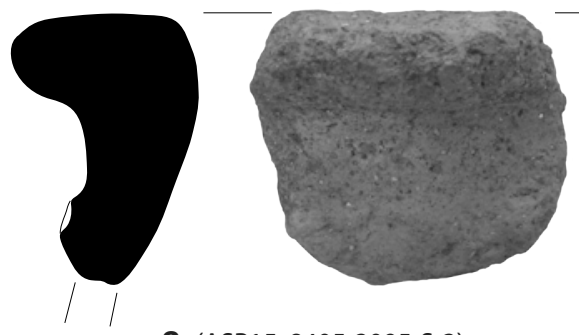

8 (ASP15; 8405-2095-S-3)

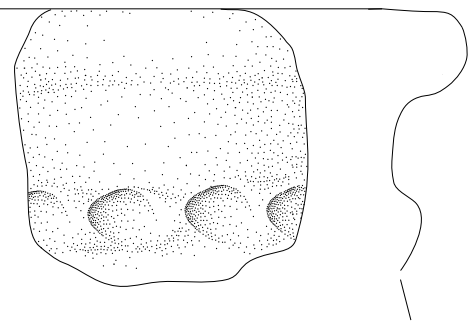



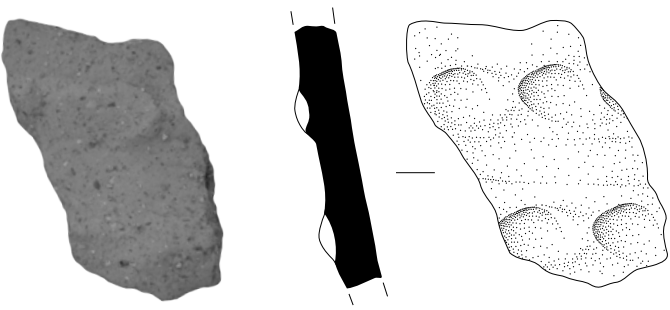

9 (ASP23; 8795-9925-S-1)

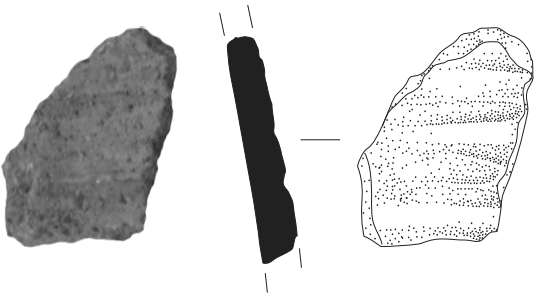

12 (ASP27; 8146-29-1-65-1)

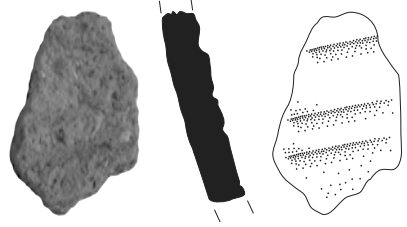

14 (ASP34; 4020-17-1-5-2)
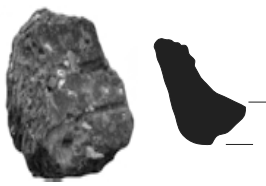

17 (ASP180; 8785-9915-S-2)
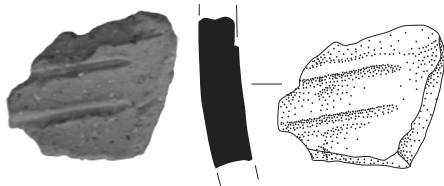

19 (ASP197; 6705-1485-S-4)

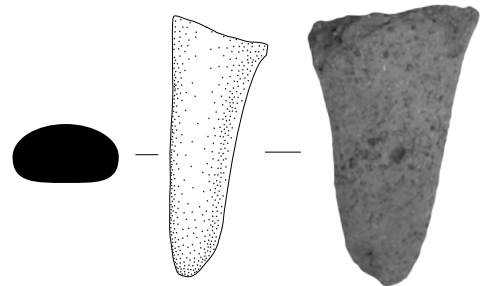

15 (ASP36; 8805-9925-S-5)

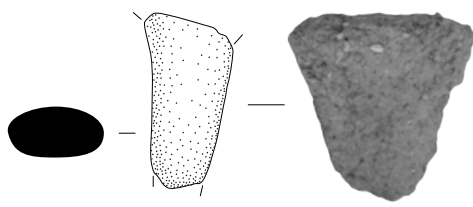

16 (ASP37; 8795-9935-V-2)
0

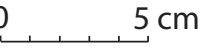

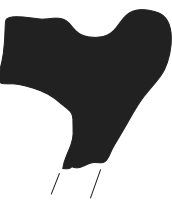

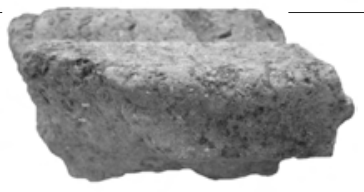

11 (ASP26; 7795-1875-S-2)
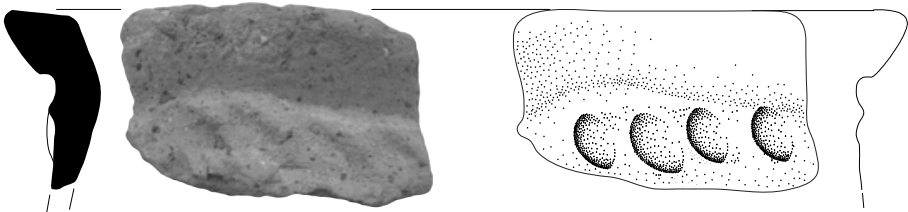

18 (ASP182; 8115-2375-S-1)
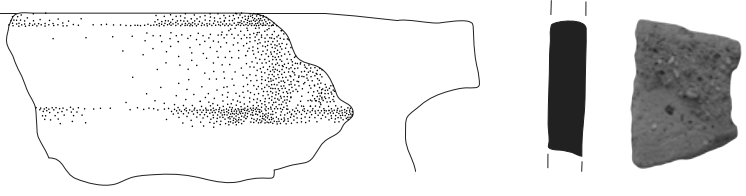

10 (ASP24; 7955-2155-V-3)
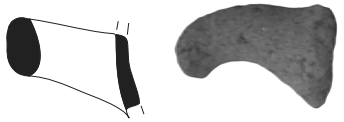

13 (ASP32; 9645-0345-S-1) 

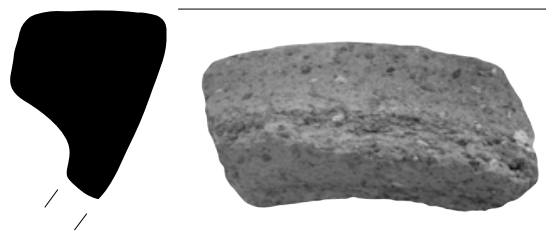

21 (ASP8; 8805-9935-S-1)
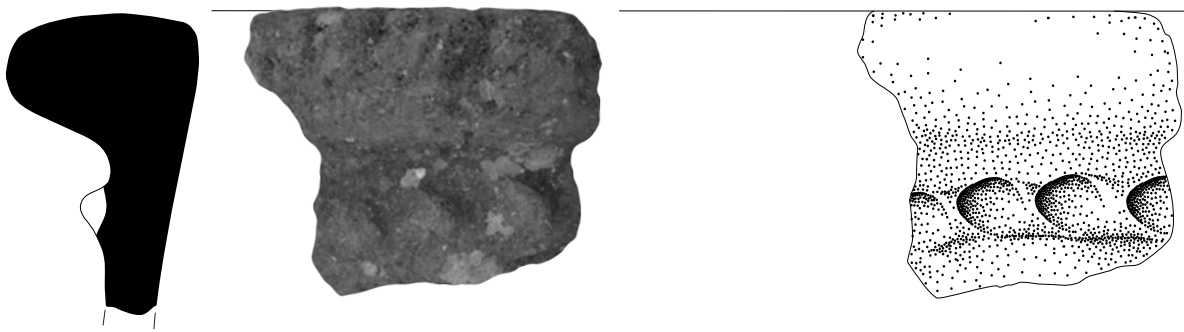

22 (ASP92; 8005-2155-S-2)

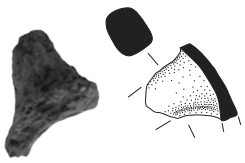

20 (ASP40; 12393-17-1-15-5)
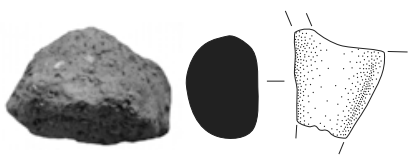

23 (ASP122; 4210-39-1-65-1)
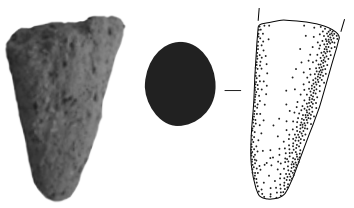

25 (ASP136; 6325-3425-S-1)
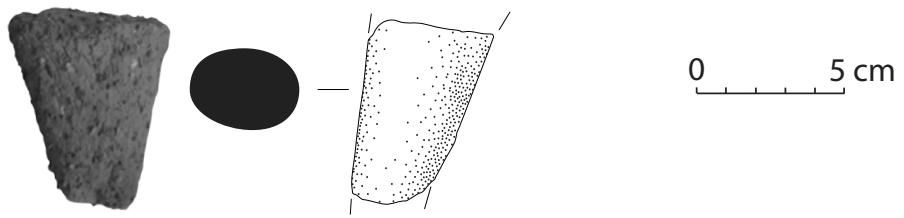

24 (ASP127; 6385-3455-S-1) 


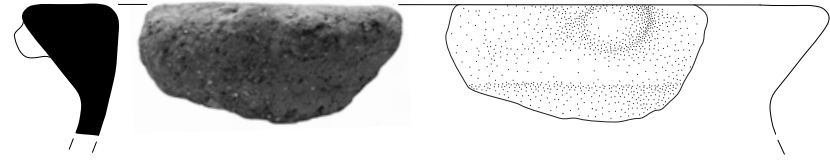

32 (ASP29; 8105-1685-S-1)
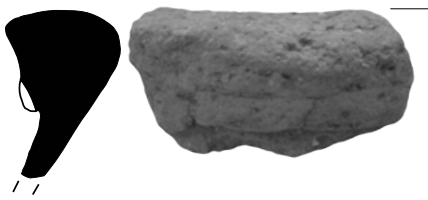

35 (ASP181; 7335-3425-S-2)

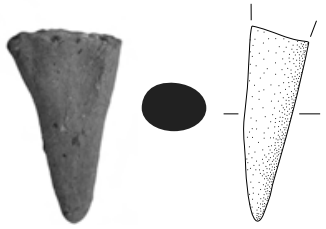

36 (ASP186; 6755-1475-S-14)
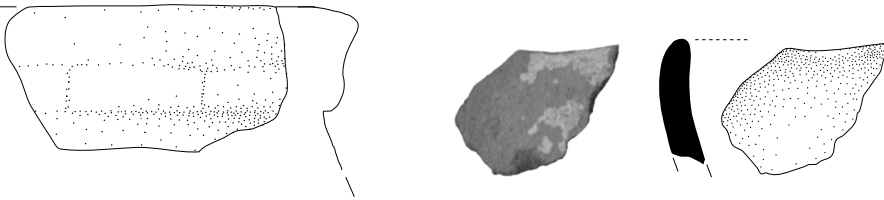

37 (ASP199; 6745-1475-V-1)
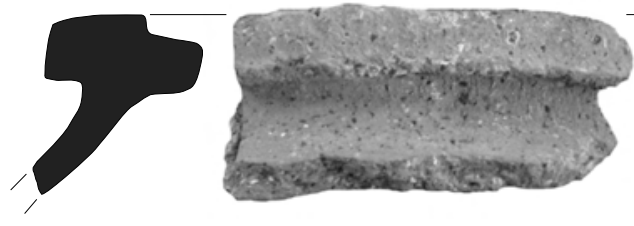

39 (ASP5; 4219-17-1-5-2)

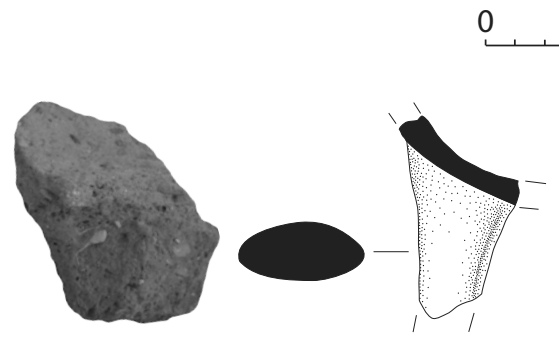

38 (ASP31; 6795-2225-S-2)
$5 \mathrm{~cm}$

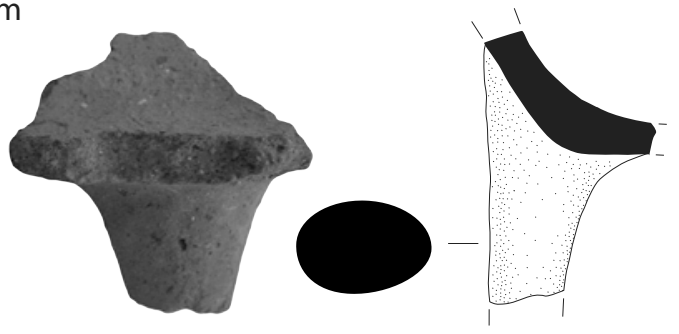

40 (ASP184; 6735-1465-S-1)
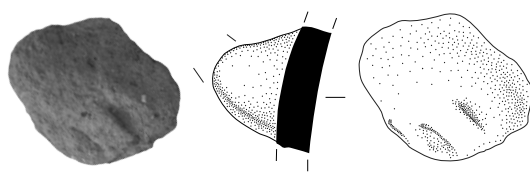

41 (ASP75; 3693-9-1-35-1)
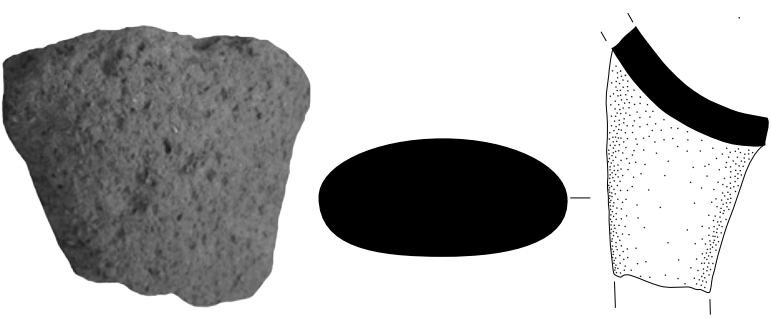

42 (ASP134; 7325-3415-S-2) 


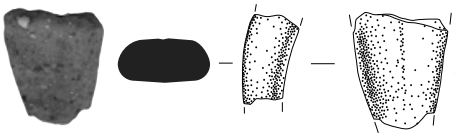

43 (ASP19; 6725-1945-S-3)
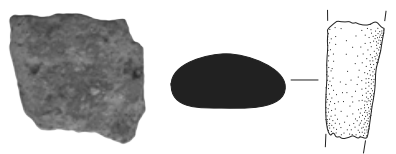

44 (ASP20; 8005-2205-S-1)
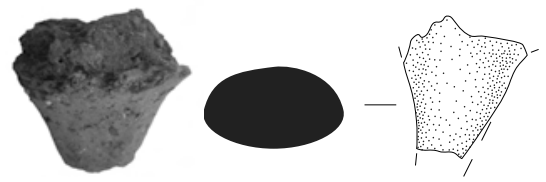

46 (ASP41; 11089-4-1-65-4)

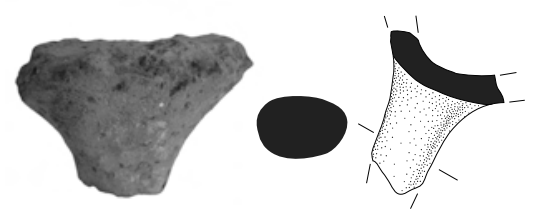

48 (ASP185; 6745-1465-S-4)

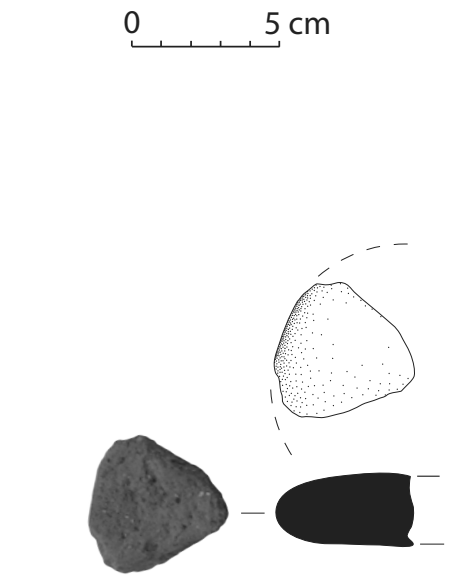

45 (ASP21; 7935-2145-S-2)
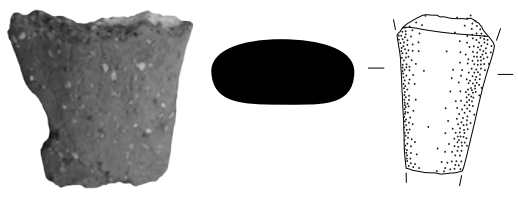

47 (ASP48; 8025-2155-V-1)

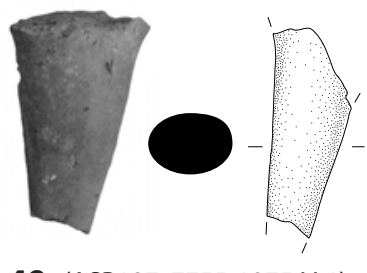

49 (ASP187; 7755-1975-V-1) 


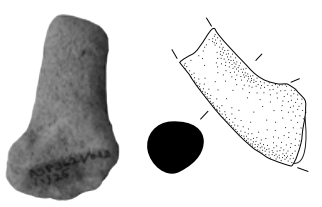

50 (ASP177; 3621-42-1-25-3)

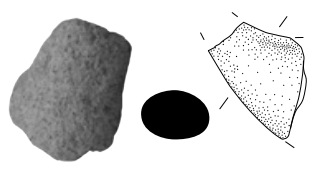

51 (ASP178; 7835-1875-S-7)

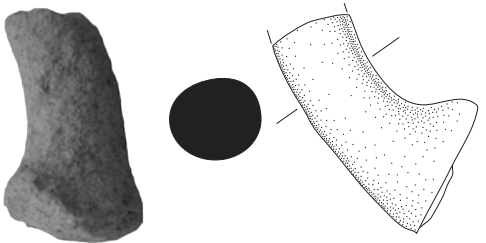

52 (ASP179; 9555-0085-S-1)
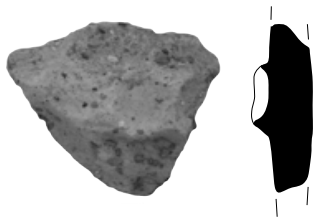

53 (ASP43; 6335-3475-S-1)

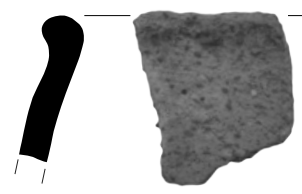

55 (ASP22; 3003-15-1-15-1)
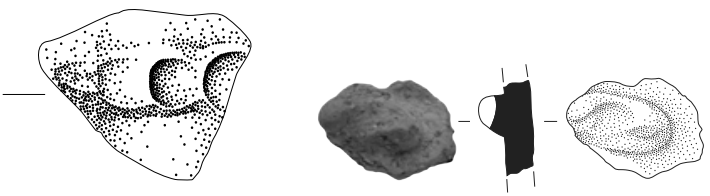

54 (ASP44; 8045-2205-S-1)

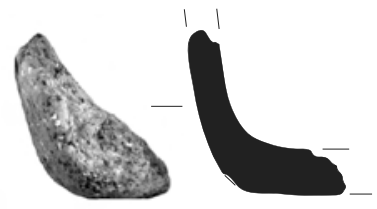

56 (ASP196; 6555-3265-S-1)

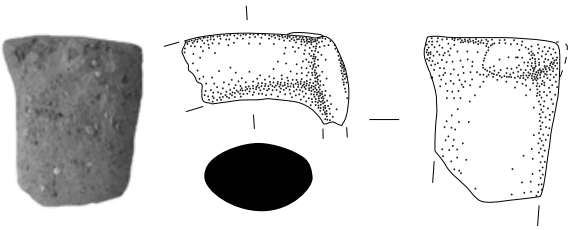

57 (ASP45; 7825-1845-S-3) 


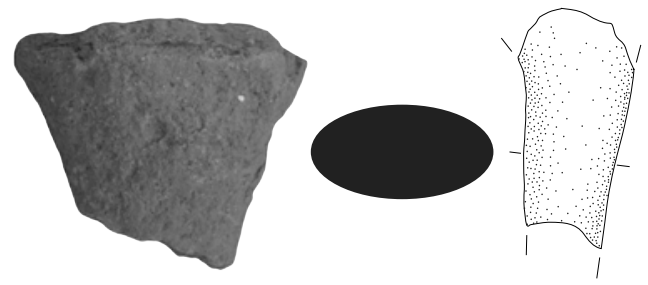

58 (ASP80; 2042-11-1-15-1)
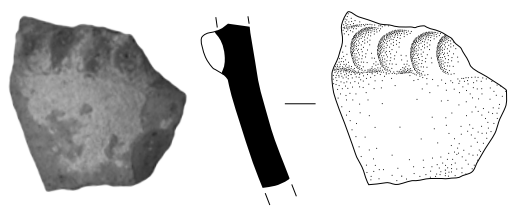

60 (ASP87; 4253-17-1-25-7)

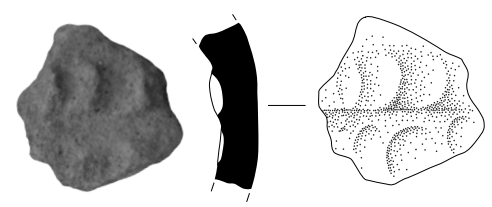

59 (ASP81; 12082-51-1-35-2)
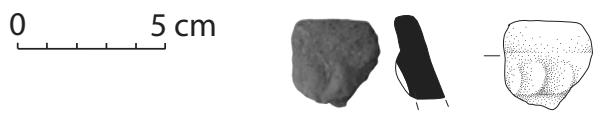

62 (ASP97; 8048-20-1-75-2)

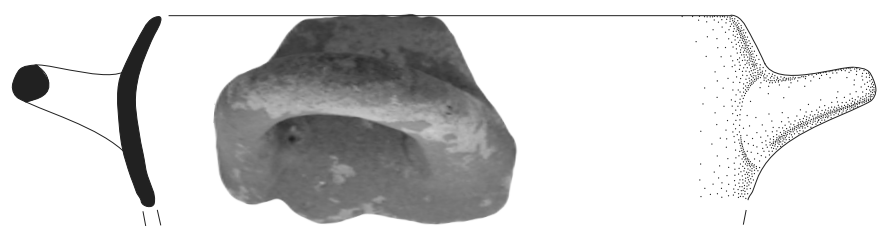

61 (ASP93; 8425-2065-S-9)

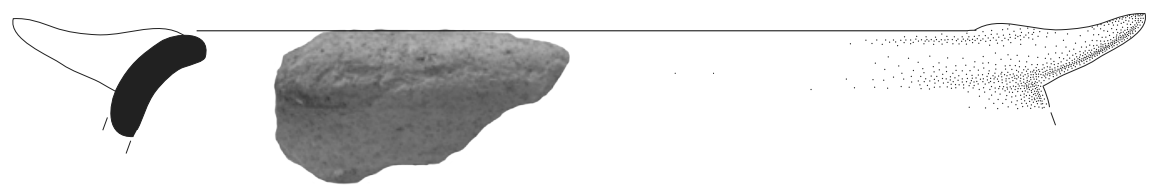

63 (ASP101; 8775-9885-V-1)

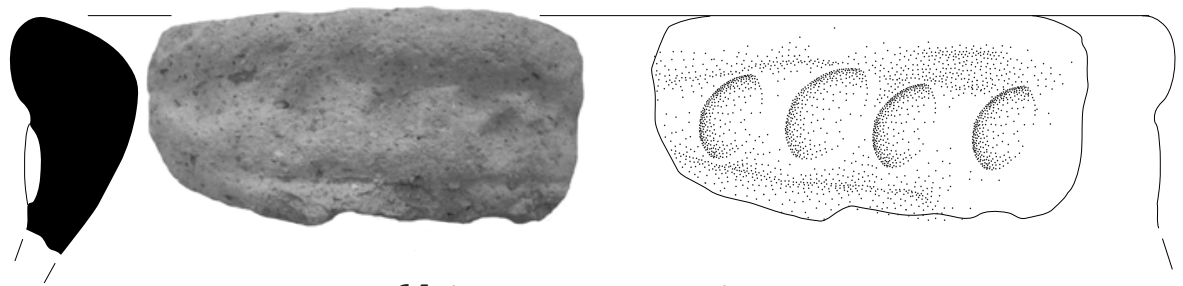

64 (ASP103; 8815-9915-S-2) 


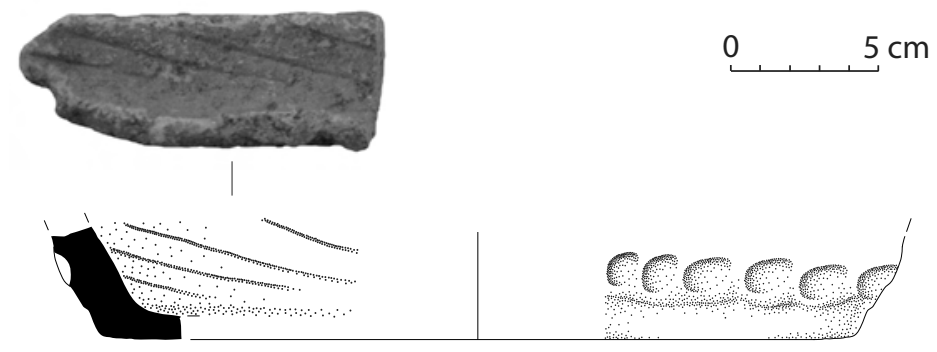

65 (ASP106; 8425-2065-S-6)

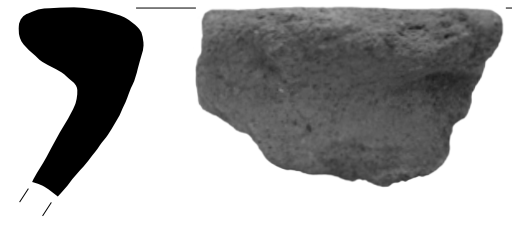

66 (ASP112; 12039-57-1-95-2)
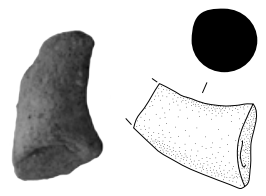

67 (ASP175; 4161-29-1-5-1)

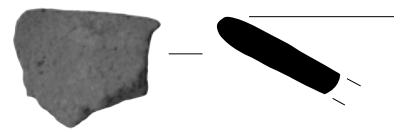

69 (ASP86; 8795-9925-S-5)

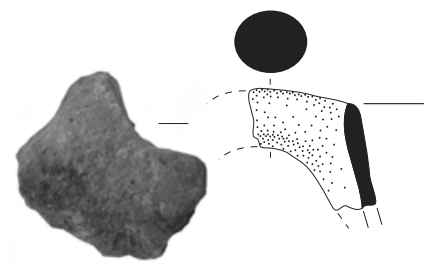

71 (ASP84; 8035-2215-S-1)

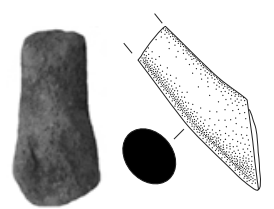

68 (ASP176; 7081-20-1-65-3)
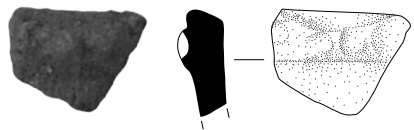

70 (ASP89; 8045-2145-S-2)

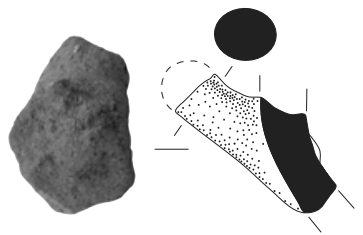

72 (ASP189; 8045-2185-S-1) 


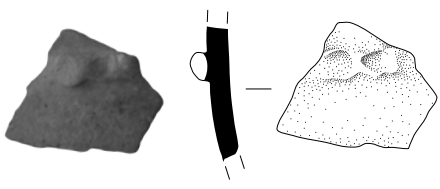

73 (ASP72; 7955-2145-V-2)

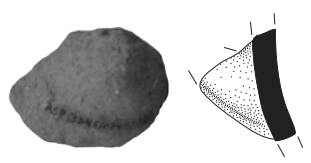

76 (ASP76; 3645-34-1-35-1)

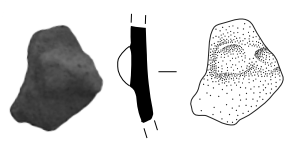

74 (ASP73; 8045-2205-S-1)

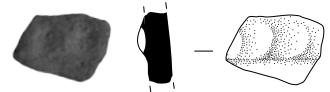

78 (ASP82; 8025-2205-S-1)

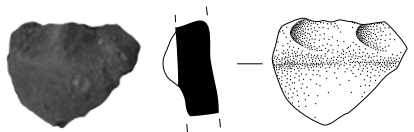

75 (ASP74; 8035-2155-S-3)

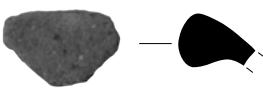

79 (ASP85; 8055-2225-S-1)

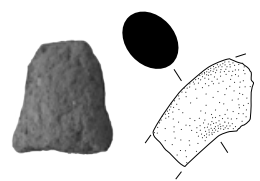

81 (ASP105; 9385-0815-S-2)

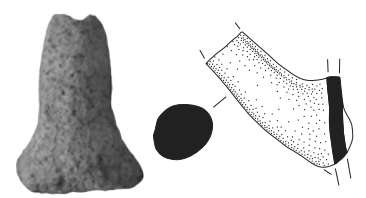

82 (ASP107; 9565-0085-S-1)

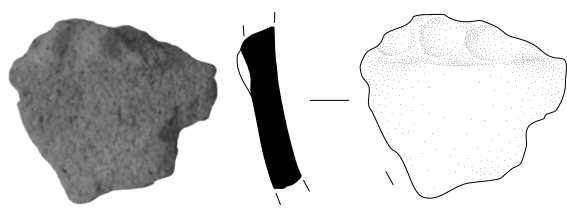

83 (ASP110; 7815-1535-S-1)
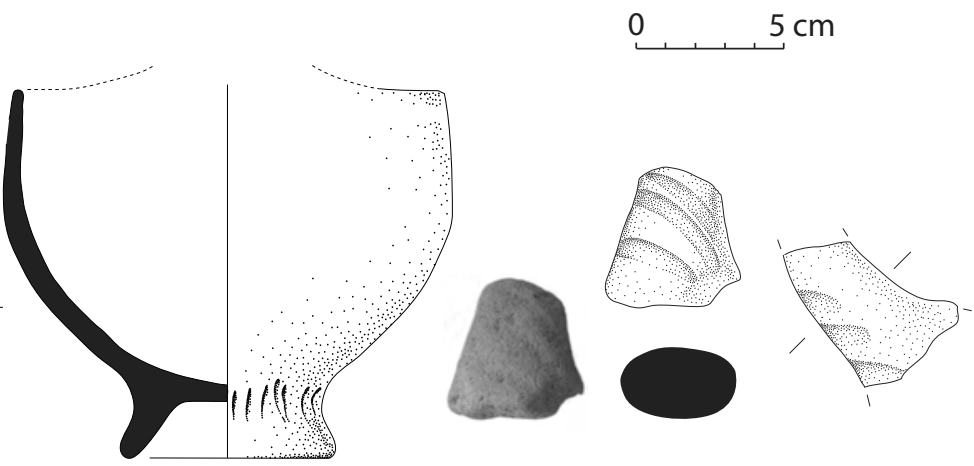

77 (ASP77; 3470-14-1-5-1)

80 (ASP102; 8815-9885-S-1) 

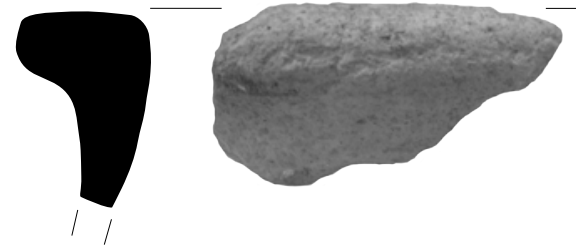

84 (ASP100; 8048-20-1-35-1)
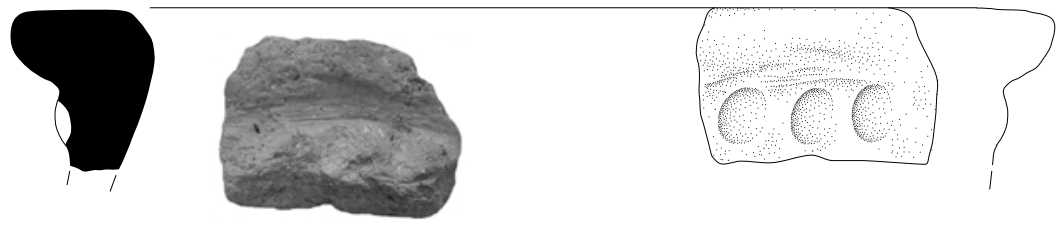

85 (ASP113; 6725-1485-S-1)

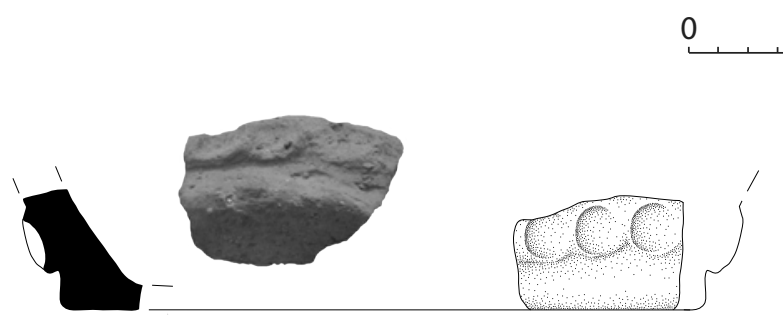

86 (ASP118; 6265-2425-S-2)

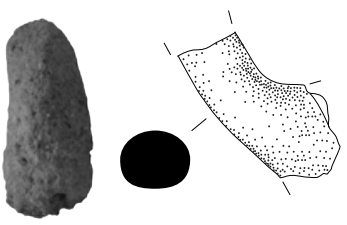

88 (ASP116; 7015-2165-S-1)
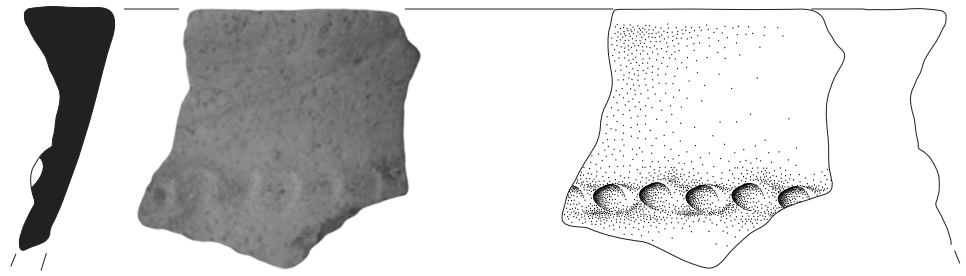

87 (ASP94; 9385-0815-V-16) 

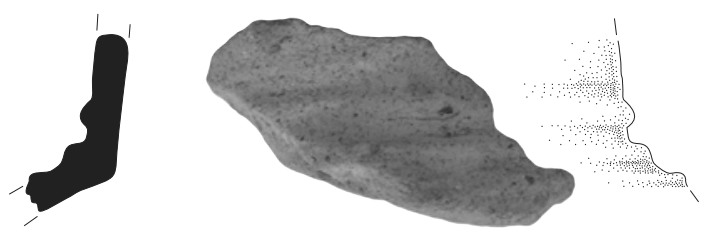

89 (ASP95; 8815-1705-S-1)
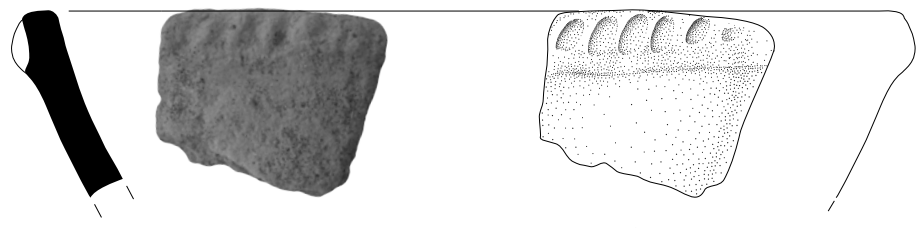

91 (ASP99; 12171-51-1-35-4)
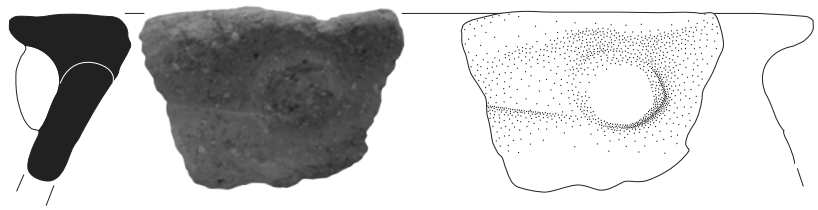

93 (ASP115; 9385-0875-S-1)
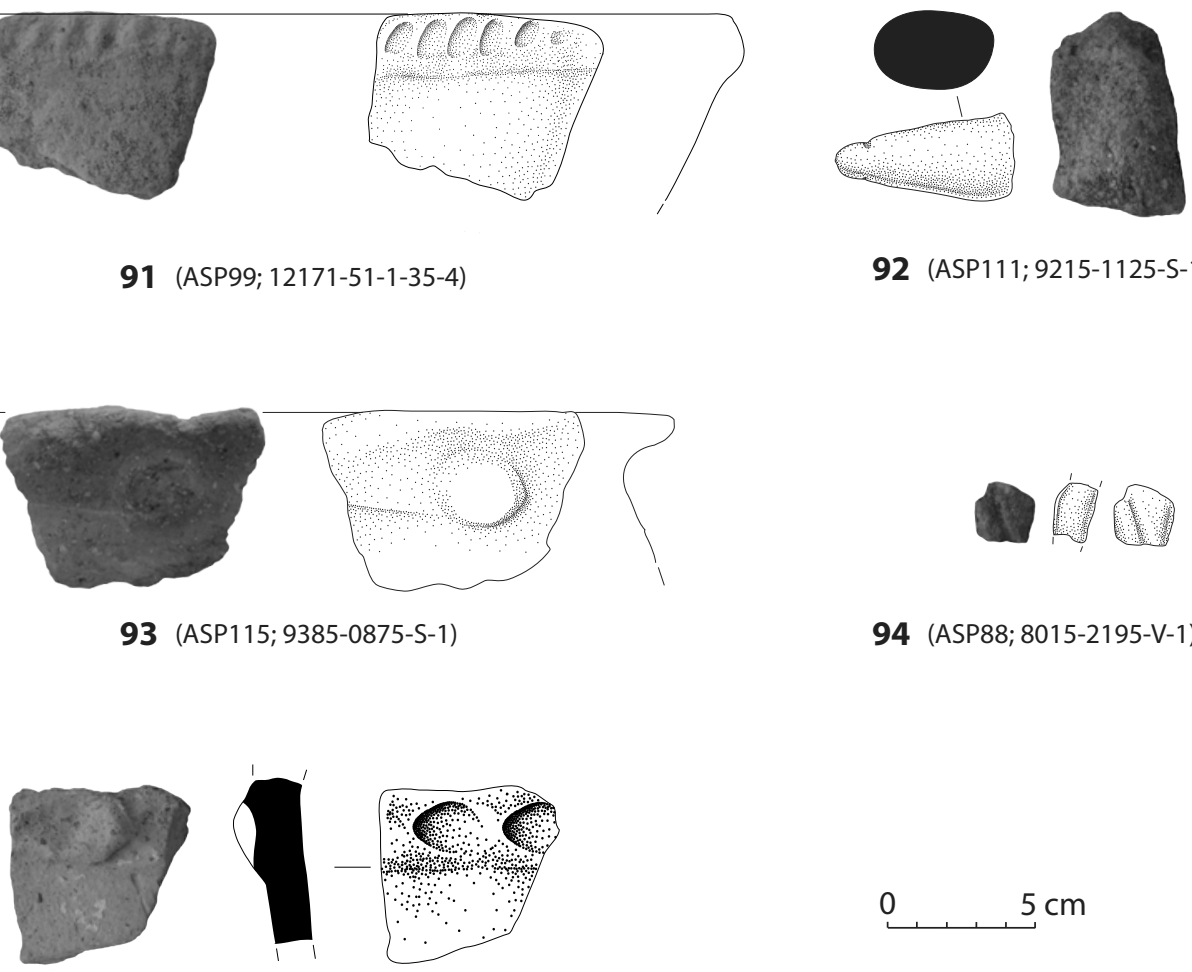

92 (ASP111; 9215-1125-S-1)

90 (ASP96; 7315-3375-S-2)

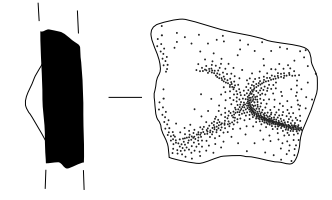



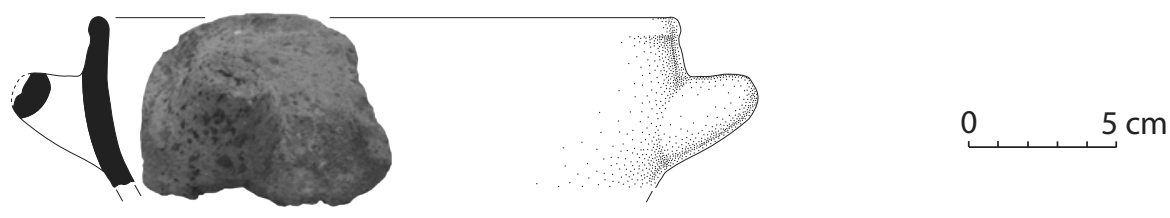

96 (ASP120; 5028-4-1-15-1)

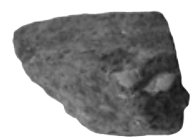

98 (ASP124; 3741-19-1-75-1)

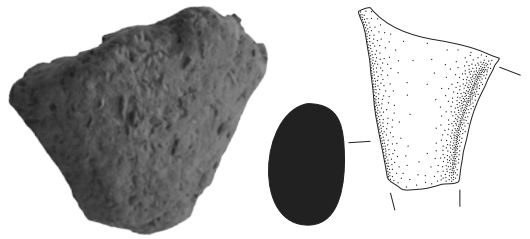

97 (ASP123; 8311-14-1-95-1)
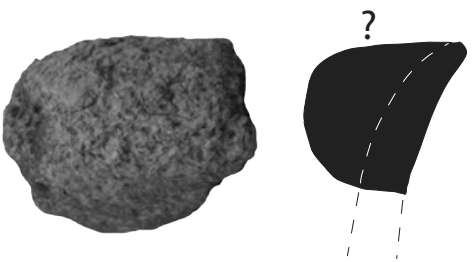

99 (ASP125; 3566-42-1-5-1)
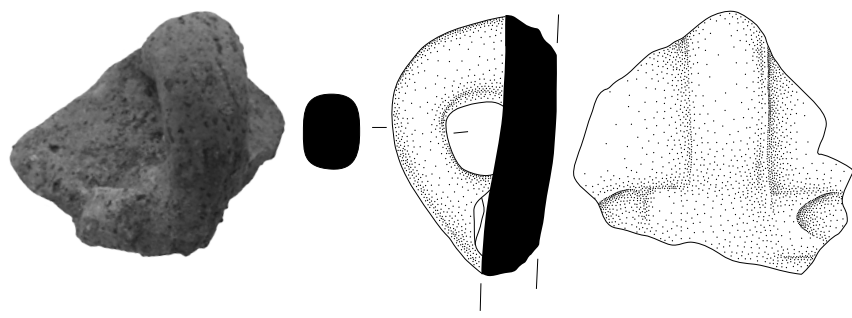

100 (ASP128; 8015-2145-S-1)
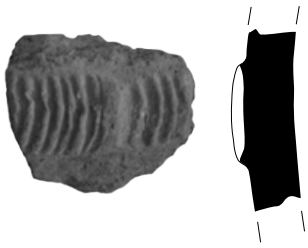

101 (ASP132; 6715-1495-V-1)

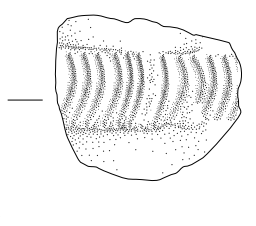

102 (ASP133; 7305-3405-S-1)
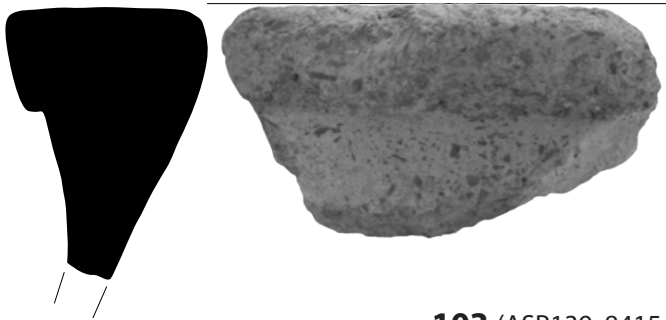

103 (ASP139; 8415-2095-S-1)

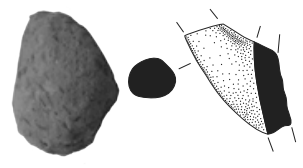



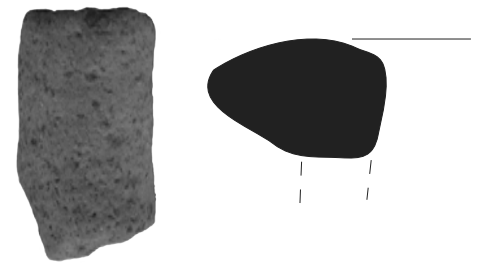

104 (ASP126; 11062-45-1-5-1)
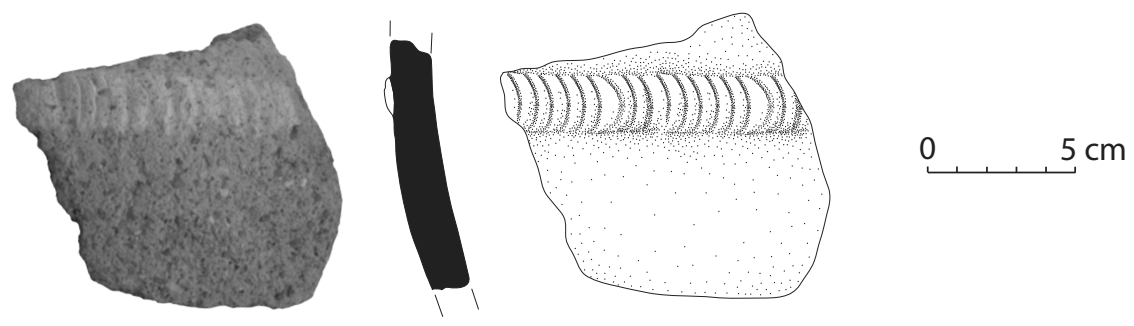

\section{5 (ASP129; 8125-2365-V-1)}
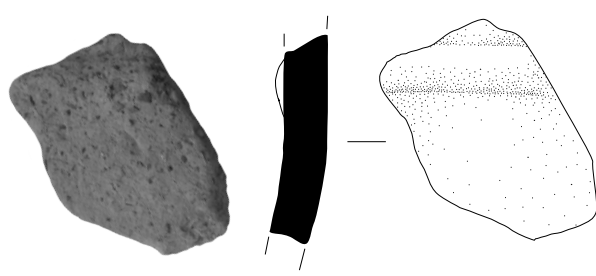

106 (ASP130; 8825-9925-S-2)
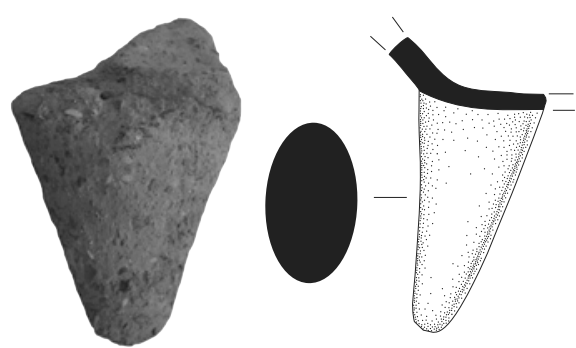

107 (ASP138; 9645-0375-S-4) 

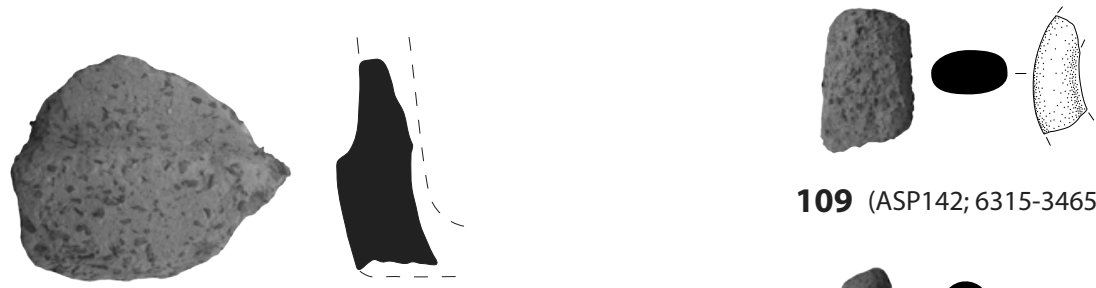

109 (ASP142; 6315-3465-S-1)

108 (ASP141; 8005-2155-V-1)
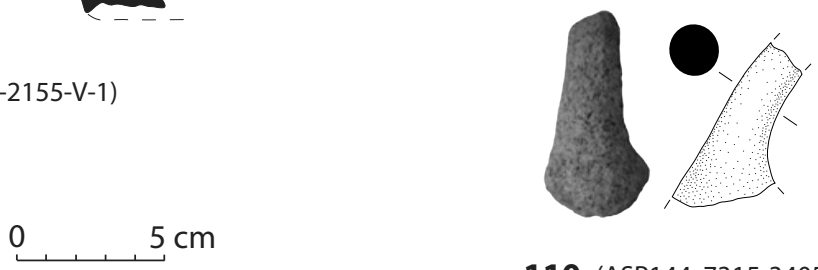

110 (ASP144; 7315-3405-S-2)
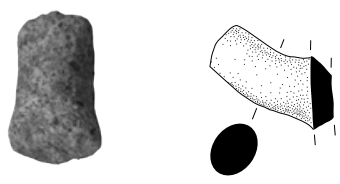

111 (ASP145; 7305-3425-S-3)
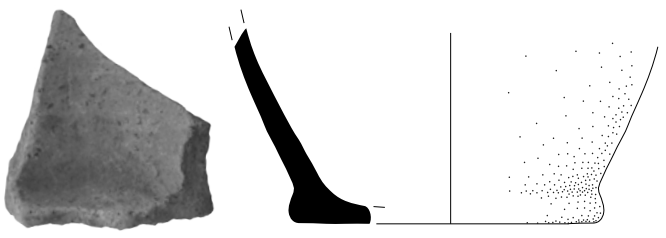

112 (ASP146; 2006-2-1-5-2)
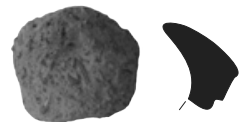

113 (ASP135; 7325-3395-S-2)

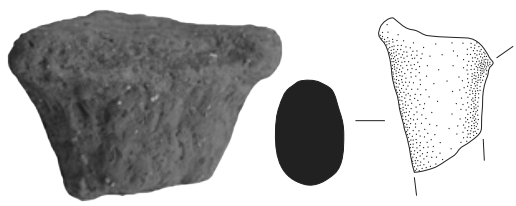

114 (ASP131; 7295-3405-S-3) 


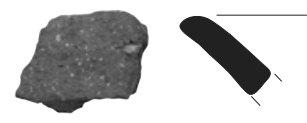

115 (ASP49; 6555-3265-S-2)
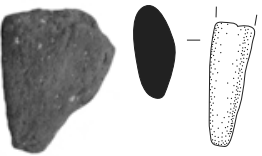

118 (ASP52; 8065-2195-S-2)

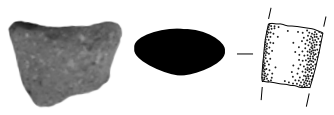

121 (ASP62; 8015-2175-S-1)


124 (ASP60; 3461-19-1-25-1)
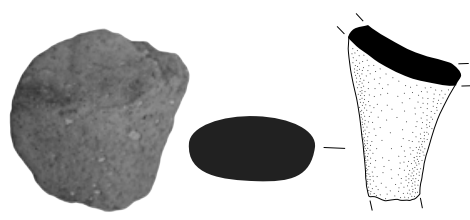

122 (ASP70; 8785-9915-S-1)
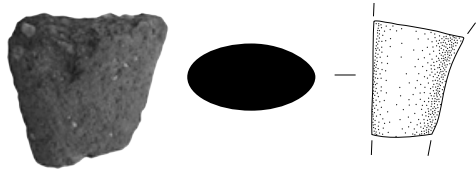

116 (ASP50; 8005-2155-S-1)

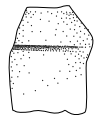

119 (ASP53; 7955-2155-V-2)
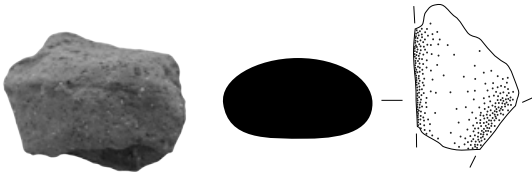

120 (ASP57; 4222-17-1-15-1)

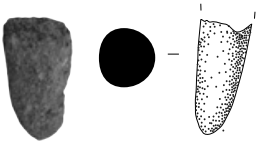

123 (ASP54; 8035-2145-S-1)
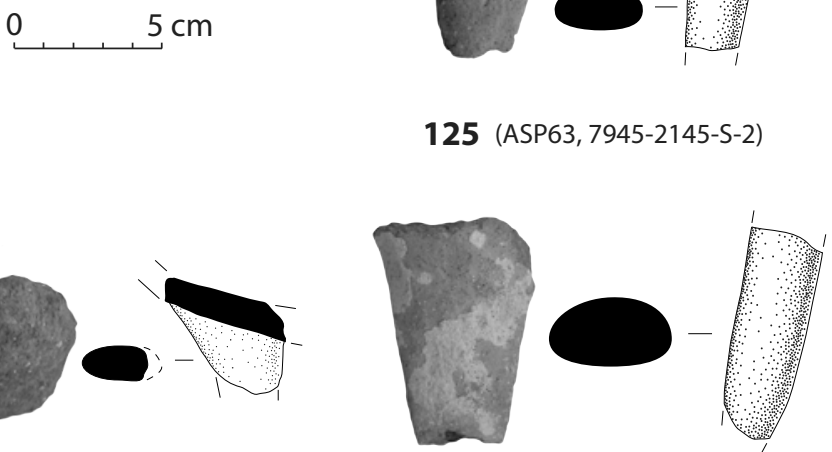

125 (ASP63, 7945-2145-S-2)

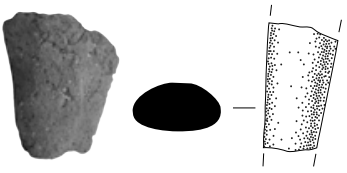

128 (ASP65; 3429-5-1-15-1)
127 (ASP71; 6795-2215-S-1)

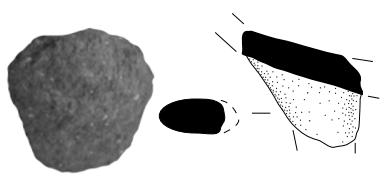

117 (ASP51; 8075-2155-V-2)

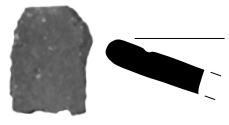

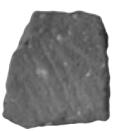

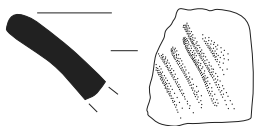

126 (ASP69; 7725-1975-V-1) 

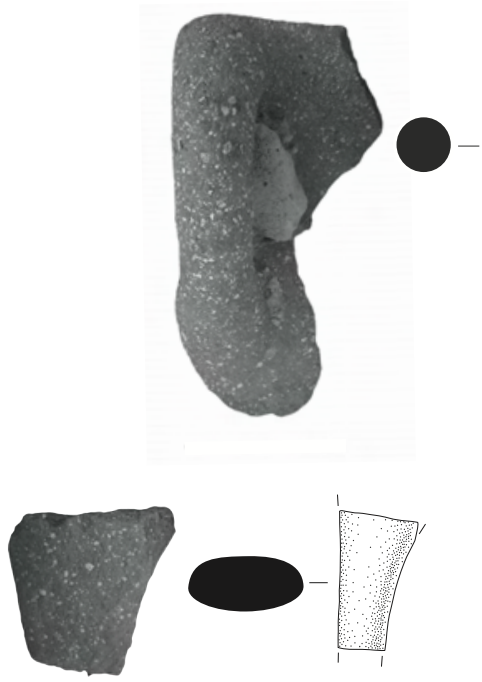

129 (ASP55; 8055-2205-S-2)

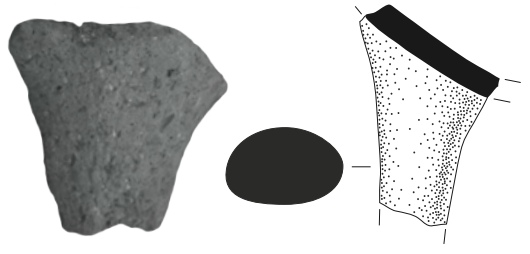

132 (ASP64; 8055-2205-S-1)
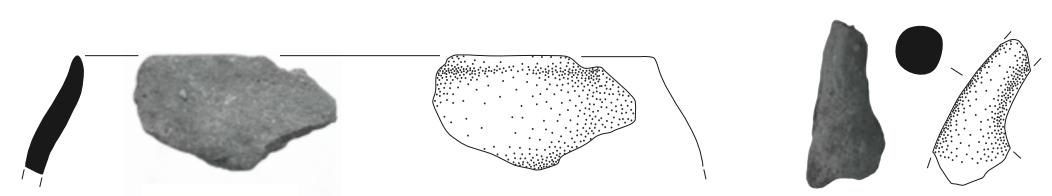

130 (ASP58 and ASP61; 6555-3255-S-3 and 4)
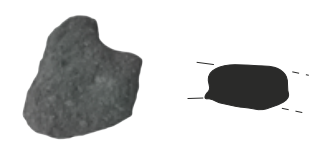

131 (ASP59; 8095-1685-S-2)

133 (ASP67; 8015-2185-V-2)

134 (ASP68; 8035-2155-S-2)
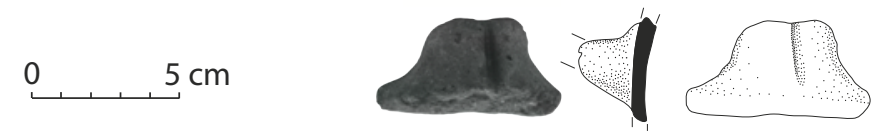

135 (ASP56; 6755-1475-S-10) 

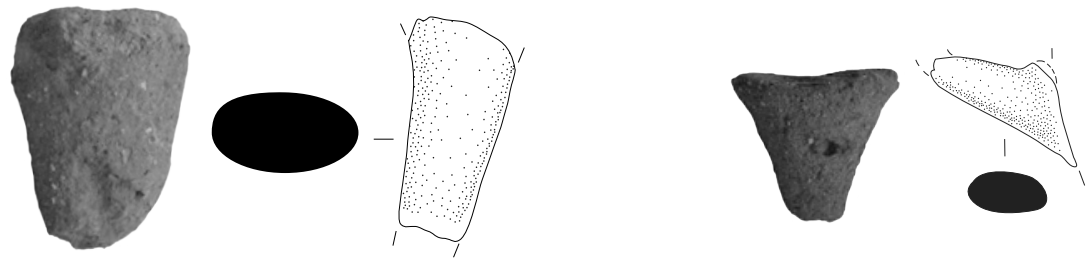

136 (ASP155; 2006-2-1-5-1)

137 (ASP158; 3235-19-1-75-1)
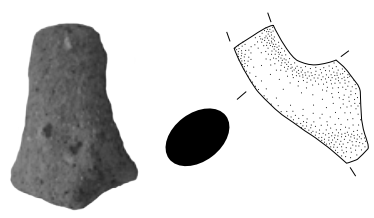

138 (ASP156; 5036-7-1-55-1)

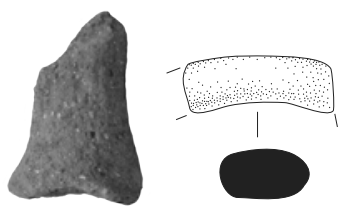

139 (ASP157; 4036-14-1-35-1)

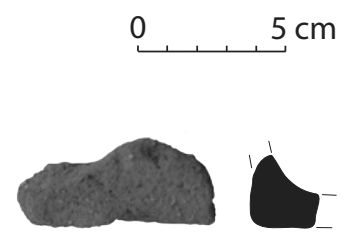

140 (ASP161; 6815-2255-S-1)
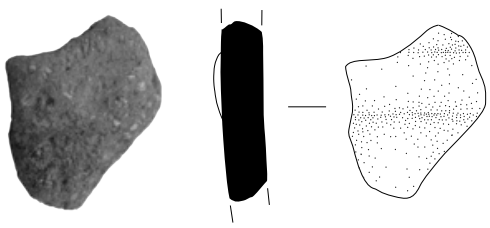

141 (ASP152; 8785-9915-S-4)

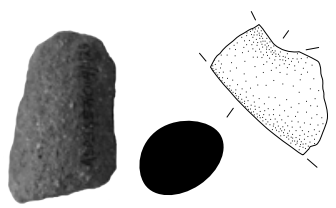

142 (ASP159; 3457-19-1-25-1)
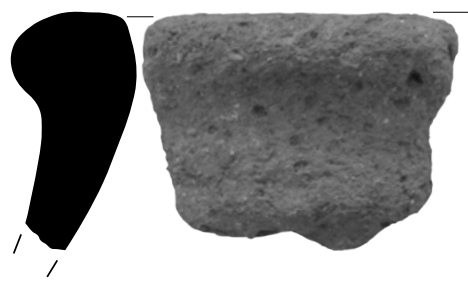

143 (ASP150; 4029-14-1-15-1)

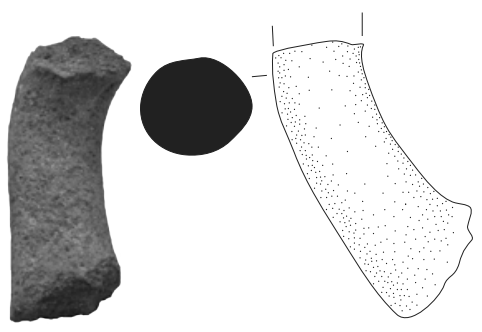

144 (ASP153; 6545-3245-S-1) 

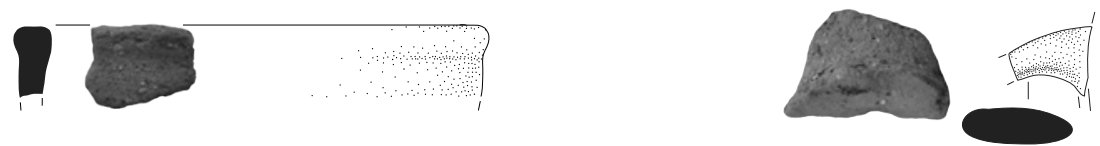

145 (ASP149; 8078-27-1-95-1)
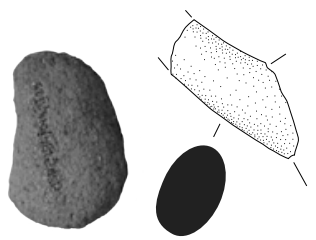

148 (ASP151; 8184-14-1-25-1)

146 (ASP160; 9395-0895-S-2)

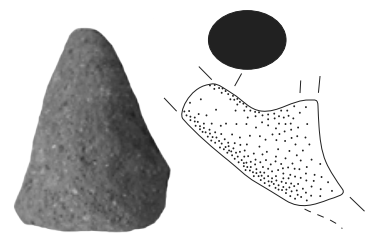

147 (ASP172; 3623-2-1-55-1)
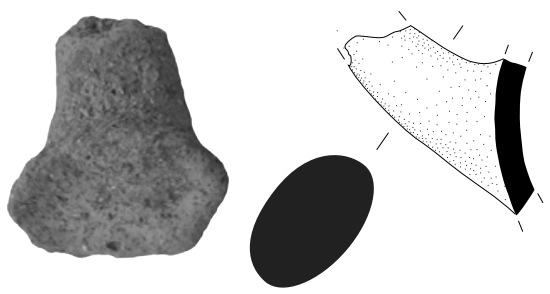

149 (ASP154; 7325-3415-S-1)
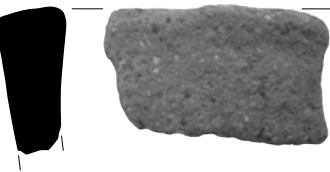

150 (ASP148; 11031-5-1-15-1)
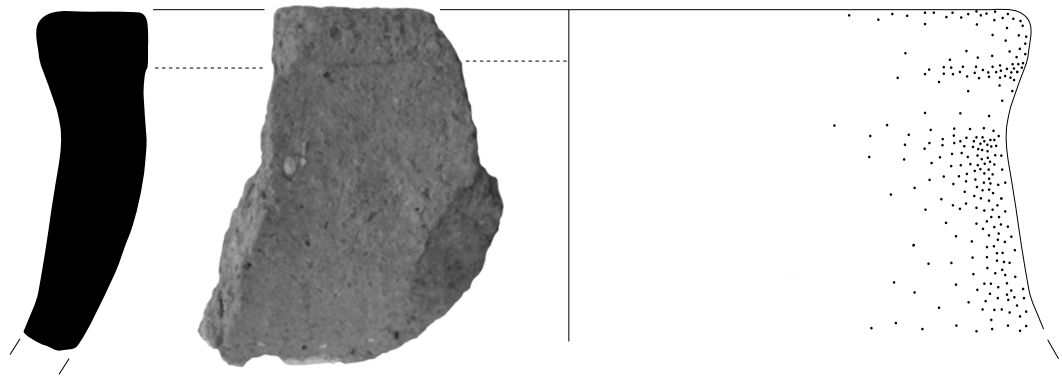

0 $5 \mathrm{~cm}$

151 (ASP165; 16013-35-1-85-1)

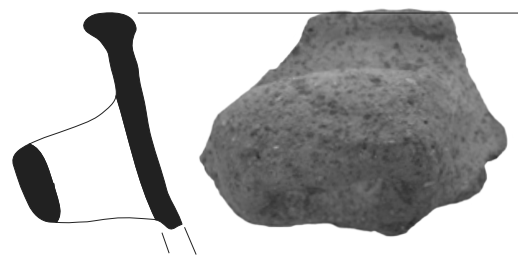

152 (ASP147; 6084-27-1-55-1) 


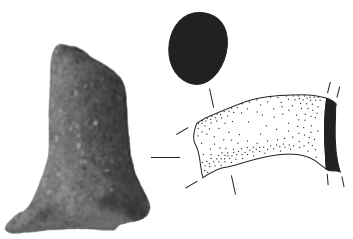

153 (ASP162; 12171-51-1-35-3)

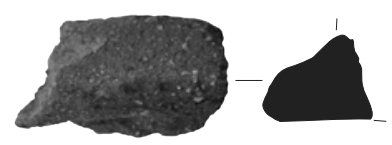

154 (ASP163; 4035-14-1-45-2)

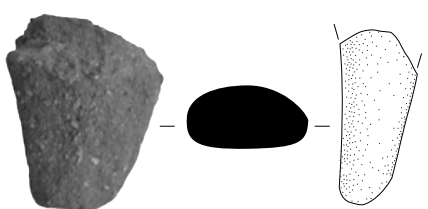

156 (ASP166; 1064-2-1-5-2)

$0, \quad 5 \mathrm{~cm}$



157 (ASP167; 7149-27-1-55-1)

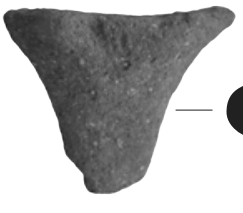

158 (ASP168; 9078-32-1-5-1)

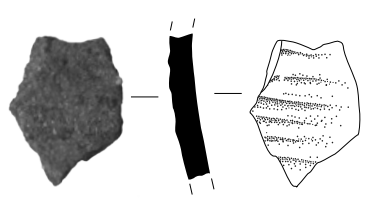

159 (ASP169; 11089-4-1-65-1)

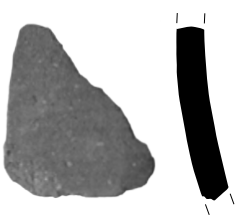

160 (ASP170; 6715-1485-V-1)
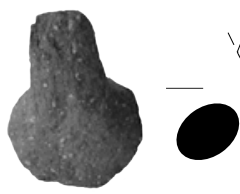

161 (ASP173; 10001-44-1-5-1)
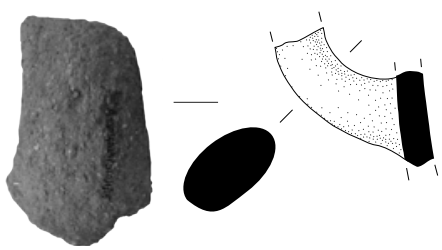

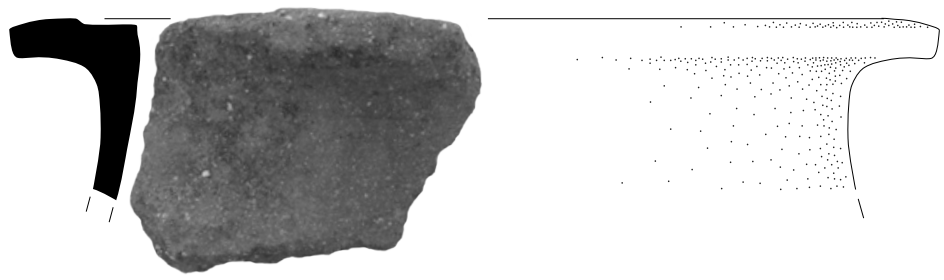

155 (ASP164; 11085-4-1-55-1)

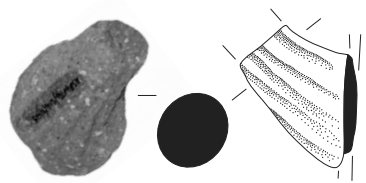

163 (ASP171; 4174-14-1-45-1) 

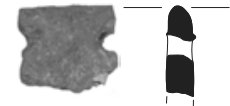

175 (7131-40-1-5-1)

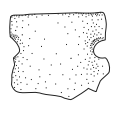

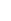

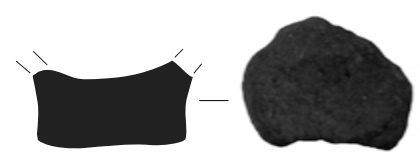

176 (9305-1535-S-1)

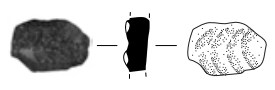

177 (8055-20-1-5-3)

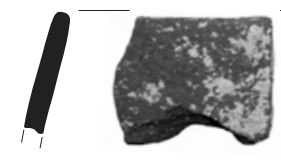

178 (7835-1885-S-1)

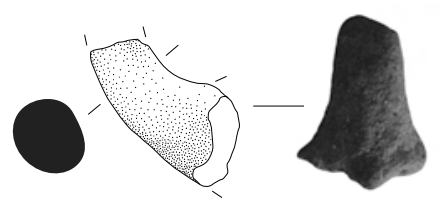

180 (3422-16-1-55-1)

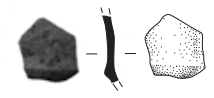

181 (6755-1475-V-7)
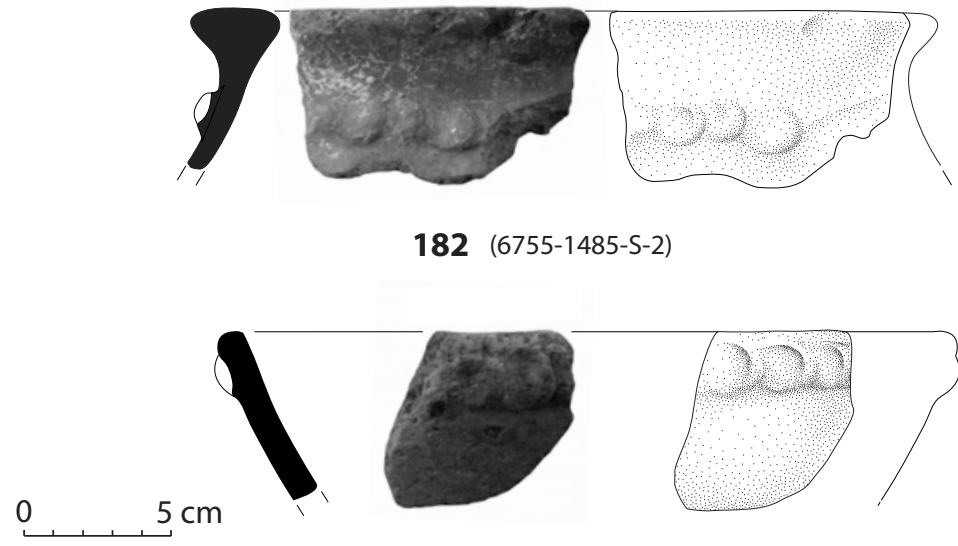

182 (6755-1485-S-2)

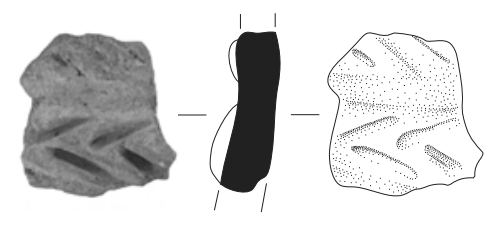

179 (7795-2145-V-3) 


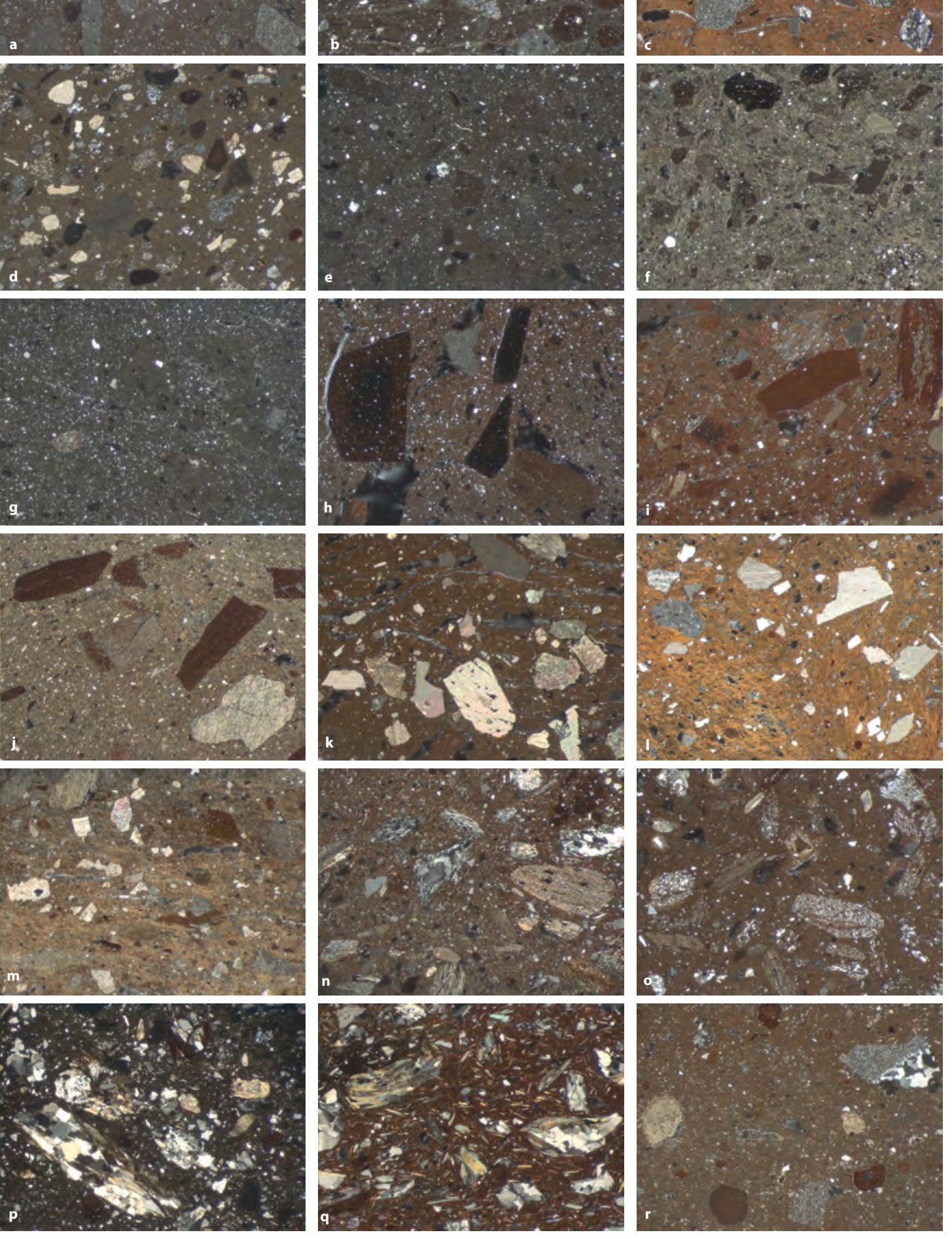

